\title{
A Especificação Formal e o Teste de um Protocolo de Comunicação de Grupo
}

\author{
Perry Roland James
}

Dissertação de Mestrado apresentada ao

INSTITUTO DE MATEMÁTICA E ESTATÍSTICA

DA

UNIVERSIDADE DE SÃO PAULO

\author{
Curso: Mestrado em Matemática Aplicada \\ Área de Concentração: Ciência da Computação \\ Orientadores: Prof. Dr. Markus Endler \\ Profa. Dra. Marie-Claude Gaudel
}

São Paulo, 1997

O aluno recebeu apoio da CAPES 


\section{A Especificação e o Teste de um Protocolo de Comunicação de Grupo}

\footnotetext{
Este exemplar corresponde à redação final da dissertação apresentada por Perry Roland James, devidamente corrigida e aprovada pela comissão julgadora.
}

São Paulo, otubro de 1997

Banca examinadora:

Prof. Dr. Markus Endler

IME - USP

Prof. Dr. Siang Wun Song

IME - USP

Prof. Dr. Jean-Marie Farines

DAS - UFSC 
to Marcia and Jailson, 


\section{Agradecimentos}

À minha família pela torcida.

À dona Lêonia e sua família pela ajuda na minha assimilação à vida no Brasil.

Ao Prof. Dr. Markus Endler e à Profa. Dra. Marie-Claude Gaudel à pela orientação segura, profissionalismo, interesse, dedicação e amizade demonstrados.

À Fundação Coordenação de Aperfeiçoamento de Pessoal de Nível Superior (CAPES) pela bolsa concedida. 


\title{
Resumo
}

Um mecanismo de comunicação de grupo é um serviço útil para a implementação de sistemas distribuídos, mas poucos trabalhos se preocuparam em especificar formalmente os protocolos usados para sua implementação. Esta dissertação descreve o protocolo de comunicação de grupo que foi implementado e os métodos formais usados. Foi implementado um protocolo de difusão de mensagens (broadcast) atômico usando um protocolo de confirmação em duas fases (two-phase commit) que seja tolerante a falhas. Este sistema foi escrito na linguagem Concert/C por causa do seu apoio para a comunicação entre processos UnIX. Inicialmente, foi desenvolvido uma especificação formal para o protocolo e mostrada a sua validade. Em seguida, casos teste para a implementação final foram gerados a partir da especificação. A implementação do protocolo foi testada usando o conjunto de testes criados para mostrar sua conformidade com a especificação original.

\begin{abstract}
A group communication mechanism is a useful service for implementing distributed systems, but little work has been done in formally specifying and testing the protocols used to implement such mechanisms. This work dissertation describes the groupcommunication protocol that was implemented and the formal methods used. A fault tolerant two-phase-commit atomic-broadcast was implemented. Concert/C was the chosen implementation language because of its communication support for UNIX processes. First a formal specification for the protocol was developed, and its validity was established. Next, test cases for the final implementation were generated from the specification. The protocol implementation was tested using the already-created test suite to show its conformance to the original specification.
\end{abstract}




\section{Sumário}

1. Introdução ............................................................................. 1

2. Propriedades dos Protocolos de Comunicação de Grupo ..................... 1

3. Modelos de Computação Distribuída ..................................................... 2

4. Alguns Exemplos de Protocolos de Comunicação de Grupo ............... 3

5. Suposiçõoes e Modelos das Camadas de Protocolos ............................. 4

6. Linguagens de Especificação Formal ................................................. 9

7. Ferramentas Disponíveis ............................................................... 12

8. A Especificação Formal .................................................................. 14

9. Validação da Especificação .............................................................. 17

10. Criação de Casos Teste ....................................................................... 20

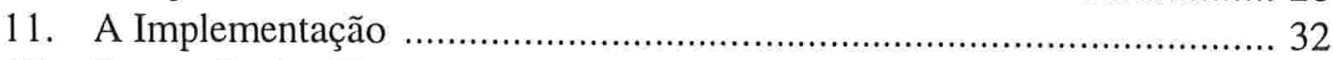

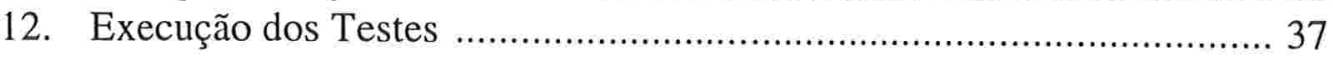

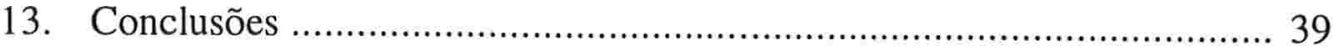

Apêndices
A. Especificação Informal da $G P E$
A-1
B. Especificação da GPE em LOTOS
A-5
C. Explicação para a Especificação Formal
A-14
D. Conjunto Original para a Validação dos Containers
A-20
E. Especificação dos Containers em PLUSS
$\mathrm{A}-22$
F. Conjunto para a Validação dos Containers
A-24
G. Manual do Usuário para GPElib
A-26
H. Parte do Arquivo list . $h$
A-29
I. Parte dos Arquivos gpetypes .h e ports.h
A-31
J. Parte do Arquivo gpeports . c
A-33
K. Parte do Arquivo gpemisc.c
A-38
L. Parte do Arquivo gpemain.c
A-41
M. Parte do Arquivo 1auncher.c
A-45

Bibliografia 


\section{A Especificação Formal e o Teste de um Protocolo de Comunicação de Grupo}

\section{Introdução}

Computação distribuída vem se tornando cada vez mais comum, apesar de ser inerentemente complicada. Ambientes de programação distribuída (tais como o proposto no projeto Sampa [End]) ajudam a superar os problemas de desenvolvimento de aplicações para sistemas distribuídos, uma vez que eles fornecem serviços básicos e abstrações de alto nível para os programadores de aplicações distribuídas. Uma parte importante desta infra-estrutura de apoio é a comunicação de grupo. Um mecanismo de comunicação de grupo permite que um grupo de processos seja tratado como uma única entidade. Os processos podem se juntar ao grupo como também deixá-lo, e as mensagens enviadas ao grupo são recebidas por todos os membros ou por nenhum deles. Esta última propriedade é obtida por um mecanismo de broadcast atômico.

Uma vez que este tipo de comunicação é um serviço básico do sistema, é necessário garantir que a implementação final esteja correta. Uma maneira de identificar os problemas é fazer uma análise de todos os possíveis fluxos de controle (walk-throughs) do pseudocódigo do algoritmo, mas é provável que usando tal método muitos erros passem desapercebidos. Uma melhor maneira de garantir a qualidade na implementação é formalizar a especificação do protocolo, provar sua validade e automatizar a verificação de que a implementação está em conformidade com a especificação.

Enquanto muitos protocolos ponto a ponto (tais como aqueles usados em níveis inferiores do modelo de protocolos da ISO/OSI) têm sido formalmente especificados e transformados em padrões internacionais, pouco disto tem sido feito com protocolos de níveis superiores, tais como os protocolos de comunicação de grupo. Os objetivos neste trabalho são:

1) escolher um protocolo adequado para a comunicação de grupo,

2) especificar esse protocolo numa linguagem formal de especificação,

3) provar várias propriedades do protocolo especificado a fim de mostrar sua correção (validação),

4) implementar o protocolo especificado e

5) testar a implementação para mostrar sua conformidade com a especificação (verificação).

\section{Propriedades dos Protocolos de Comunicação de Grupo}

A propriedade mais importante de um broadcast é que este seja atômico, isto é, que ou todos os processos no grupo recebem a mensagem ou nenhum deles a recebe. Outra propriedade importante é que o protocolo deve ser suficientemente robusto para suportar qualquer combinação de falhas dos processos, das máquinas e da rede. Grupos devem ser dinâmicos, ou seja, os processos devem ser capazes tanto de se juntar a um grupo quanto deixá-lo a qualquer momento.

O recebimento de mensagens para o grupo também tem que respeitar certas propriedades de ordenação. Há três tipos básicos de ordenação da entrega de mensagens associados com broadcasts: FIFO, 
causal e global. A ordenação FIFO garante que cada mensagem enviada por um mesmo processo será recebida por todos os outros processos na ordem na qual foram enviadas. Essa é uma condição fraca, uma vez que ela não dá garantias sobre a ordem das mensagens enviadas por diferentes processos. Em outro extremo, a ordenação global ou absoluta garante que todos os processos receberão todas as mensagens na mesma ordem. A ordenação causal se situa entre as duas anteriores em termos de requisitos e das propriedades que oferece. Ela garante que nenhuma mensagem seja recebida até que todas as mensagens que podem ter causado esta mensagem tenham sido recebidas. Desta forma, todas as mensagens sem uma relação de casualidade podem ser vistas como tendo sido enviadas ao mesmo tempo e podem ser recebidas em qualquer ordem. Elas podem ser entregues em qualquer ordem para os processos membros do grupo e a ordem de entrega pode ser diferente para cada membro.

Os dois possíveis tipos de semântica de broadcast são no máximo uma vez (at most once) e no mínimo uma vez (at least once). A entrega no máximo uma vez significa que o sistema tentará entregar uma mensagem do broadcast, garantindo que nenhum membro receberá a mesma mensagem duas vezes. Escolhemos um sistema com a entrega no mínimo uma vez já que queremos um sistema que garanta que todos os membros do grupo, eventualmente, recebam todas as mensagens aceitas pelo grupo. Dessa forma, seria necessário provar, também, uma maneira de detectar e tratar as mensagens duplicadas.

\section{Modelos de Computação Distribuída}

Hadzilacos e Toueg [Had] dão um resumo abrangente de vários aspectos do problema de broadcast tolerante à falha com semântica at most once. Embora a semântica dos broadcasts que eles discutem não seja compatível com o tipo com o qual estamos trabalhando, as observações feitas por eles na introdução sobre a seleção de um modelo de computação distribuída são aplicáveis.

Trabalharemos num ambiente no qual os processos se comunicam através de envio de mensagens usando uma rede, ao invés do uso de uma memória compartilhada. Uma vez que estamos interessados em especificar somente as propriedades de alto nível, assumiremos várias propriedades da rede. Ela proverá uma transmissão confiável de mensagens de qualquer tamanho entre quaisquer dois processos no sistema. O sistema é assíncrono. O único uso explícito de tempo é para timeouts que são usados para detectar processos que apresentam uma falha. Depois de mandar uma mensagem, um processo assumirá que uma mensagem não foi recebida se uma confirmação (acknowledgment) não for recebida dentro de um dado intervalo de tempo.

Há três tipos de erros que são possíveis no sistema: aqueles que são tratados por outras partes do sistema, aqueles que são tratados pelo mecanismo de comunicação de grupo e aqueles que não podem ser tratados pelo nosso sistema. Hadzilacos e Toueg classificam os modelos de falha pela severidade. Um modelo é considerado mais severo que outro se o grupo de comportamentos faltosos que ele permite é um subconjunto próprio do grupo do outro. Dado que estamos assumindo uma rede confiável, a perda de mensagens pela rede é tratada por uma outra parte do sistema. Falhas arbitrárias (ou Bizantinas), isto é, falhas nas quais um processo pode exibir um comportamento qualquer não serão tratadas pelo nosso sistema. Os seguintes tipos de falhas são tratados pelo nosso sistema:

1) Parada (Crash): um processo funciona corretamente até um ponto e, então, pára prematuramente.

2) Omissão de mensagens: um processo pára, ou algumas vezes deixa de enviar ou receber mensagens, ou ambos.

3) Falha de temporização: o tempo necessário para transmitir uma mensagem excede o seu limite. 
Na lista acima, um Crash é o menos severo e cada modelo sucessivo é mais severo do que o anterior. Os três modelos de falha listados acima são chamados benignos uma vez que é relativamente fácil detectar sua ocorrência. Nosso protocolo deve ser capaz de tratar qualquer combinação de falhas benignas.

\section{Alguns Exemplos de Protocolos de Comunicação de Grupo}

Existem vários sistemas operacionais e ambientes de programação distribuídos, provendo um mecanismo de comunicação de grupos já existem. Abaixo são resumidos os protocolos usados em Amoeba, ISIS e Horus.

\subsection{Amoeba}

O modelo computacional usado em Amoeba [Tan] é aquele no qual os processos se comunicam por meio do envio de mensagens usando uma rede que provê um broadcast não confiável de mensagens sem limitações de tamanho. Timeouts são possíveis. O sistema deve tolerar qualquer combinação de falhas benignas.

O envio de mensagens em Amoeba é usualmente feito por meio de um mecanismo de chamada remota de procedimentos (remote procedure call, ou RPC). Os grupos são coleções de processos que trabalham juntos ou para atingir um objetivo comum ou para operar como algum tipo de servidor. Todos os grupos são fechados. Quando um cliente quer se comunicar com um servidor, ele o faz por $R P C$. Se o servidor estiver implementado por um grupo, a $R P C$ é para um membro do grupo. Este membro pode usar o mecanismo de comunicação de grupo para prover o serviço ao pedido.

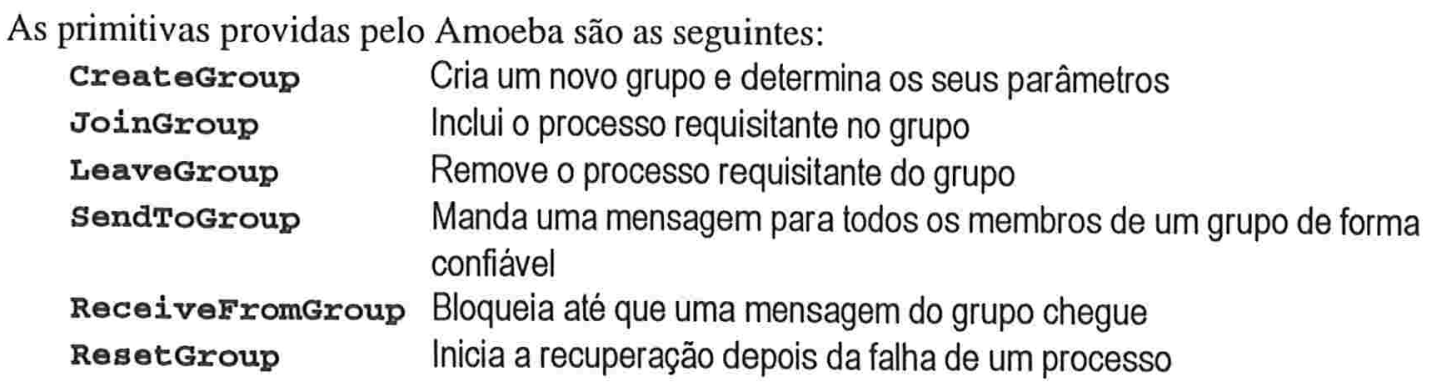

Creategroup tem como um dos seus parâmetros um fator de resiliência, $k$, que especifica o número de processos que podem falhar, antes que o grupo como um todo seja considerado falho. Ele retorna o identificador do grupo que é usado para referenciar um grupo especifico. JoinGroup e LeaveGroup incluem, como um dos seus parâmetros, uma mensagem a ser enviada a todos os membros do grupo, de modo que todos os membros possam saber quais processos estão no grupo. Um grupo é destruído quando o último membro sai. ResetGroup é usado para a recuperação de falhas. SendToGroup é usado para fazer um broadcast atômico com uma ordenação global. SendToGroup e ReceiveFromGroup bloqueiam até que a operação seja completada ([Tan]).

A ordenação global provida por SendToGroup é realizada usando um seqüenciador. Este seqüenciador é um processo auxiliar, único no sistema. SendToGroup, em vez de disseminar a mensagem para os membros diretamente, envia a mensagem para o seqüenciador. Este dá à mensagem o próximo número de sequiência disponível e dissemina a mensagem para o grupo. Já que só há um seqüenciador, o método de atribuir números de seqüência é serial. 


\subsection{Isis}

Isis ([Tan] e [Biv]) é um pouco mais flexível que Amoeba, no sentido de prover duas formas de broadcast que são diferenciadas pelo modo em que as mensagens são ordenadas. As primitivas de broadcast são ABCAST, para um broadcast com ordem total, e, CBCAST, para um broadcast com ordem causal. A ordenação global provida em Isis não é realizada por meio de um processo centralizado, como em Amoeba. A ordenação e broadcast atômico são realizados com um mesmo protocolo de duas fases. Um processo que inicia um ABCAST dissemina a mensagem para os membros do grupo. Quando os membros do grupo recebem a mensagem, estes incluem na confirmação um possível número de sequiência que é maior que os números já conhecidos pelos processos. O processo iniciador do broadcast coleta os números de seqüência sugeridos pelos membros e escolhe o maior para ser o número de seqüência da mensagem. CBCAST, o broadcast com ordenação causal, usa um vetor de timestamps para a ordenação de mensagens.

\subsection{Horus}

Horus ([Biv]) oferece muito mais flexibilidade. Ao invés de prover para os usuários poucas opções fixas para a comunicação, Horus permite que cada grupo possa configurar sua pilha de protocolos própria, por meio da combinação de microprotocolos como a conexão dos blocos de Lego. Esta flexibilidade de configuração é obtida fazendo com que todos os microprotocolos tenham a mesma interface. Os microprotocolos mais interessantes estão resumidos abaixo:

$\begin{array}{ll}\text { COM } & \text { provê a interface para os protocolos de nivel mais baixo, tais como IP } \\ \text { NAK } & \text { provê uma transmissão confiável por meio de confirmações negativas e retransmissões } \\ \text { FRAG } & \text { provê a fragmentação e remontagem de mensagens } \\ \text { MBRSHIP } & \text { fornece aos membros uma lista de receptores possiveis } \\ \text { FC } & \text { provê controle de fluxo } \\ \text { TOTAL } & \text { provê uma ordenação total de mensagens } \\ \text { STABEL } & \text { indica quando uma mensagem já foi mandada para todos os receptores } \\ \text { CRYPT } & \text { provê cifragem e decifragem }\end{array}$

Nem toda combinação de microprotocolos faz sentido e nem todo microprotocolo pode ser usado em qualquer nível na pilha. Por exemplo, a camada COM é quase sempre a camada mais baixa, enquanto que, entre as FRAG e NAK, não faz sentido usar a primeira abaixo da segunda.

O microprotocolo que provê broadcast atômico, entre outros serviços, é o MUTS (MUlticast TransportService). Ele provê um modelo de envio de mensagens que é assíncrono e um para muitos (one to many). MUTS é um esqueleto que descreve uma interface e este microprotocolo pode ser instanciado com protocolos que são confiáveis ou não, um a um (peer to peer) ou multicast. O protocolo padrão provê uma ordenação $F I F O$ e at most once, por meio de multicast confiável.

\section{Suposições e os Modelos das Camadas de Protocolos}

Como já foi previamente especificado, o modelo computacional usado é aquele no qual os processos se comunicam por meio de envio de mensagens usando uma rede que provê uma transmissão confiável entre quaisquer dois processos de mensagens de tamanho ilimitado. O único requisito temporal é que se conheça o tempo máximo para que uma mensagem seja enviada e confirmada. O sistema somente apresentará falhas benignas, isto é, falhas arbitrárias não são tratadas. 
Para ser utilizado por usuários finais, o mecanismo de comunicação de grupo deve ter um conjunto completo de primitivas, como descrito por Endler e D'Souza [End]. O objetivo neste trabalho não é implementar um sistema completo, mas, ao invés disso, implementar e testar apenas uma parte desse sistema. Para explicar a parte que interessa, é preciso dar uma descrição do serviço como um todo. As seguintes propriedades são desejáveis para um mecanismo completo de comunicação de grupo.

1) Os grupos são criados dinamicamente. Qualquer processo pode criar um grupo. O procedimento para criar o grupo terá como parâmetros o número máximo de membros no grupo, o número máximo de mensagens a serem guardadas no registro de mensagens $(\log )$, a fração mínima no número de membros do grupo para a qual a mensagem tem que ser entregue e a demora para um timeout. O procedimento devolverá o identificador do grupo.

2) Os processos podem se juntar a um grupo ou deixar um grupo dinamicamente. Os procedimentos para entrar ou sair de um grupo terão como parâmetros os identificadores do grupo e do processo remetente. Eles devolverão true para sucesso e false para falha.

3) Os Grupos são fechados. Somente membros de um grupo podem enviar mensagens para o grupo. Processos que não pertencem a um grupo comunicam-se com ele, enviando uma mensagem para um dos membros, o qual pode, então, comunicar-se com o grupo.

4) Qualquer membro de um grupo pode difundir mensagens para o grupo. Para fazê-lo, este usa um procedimento que tem como parâmetros o identificador do grupo, a mensagem e o tamanho da mensagem. O sistema bloqueia o procedimento de envio apenas por um tempo suficientemente longo para registrar o pedido de broadcast. O procedimento devolverá true para sucesso e false para falha. True não significará que a mensagem foi enviada com sucesso. Isso apenas significa que o sistema será capaz de continuar processando o pedido após recuperar-se de uma falha. Se uma mensagem é enviada com sucesso para o grupo, esta também será recebida pelo remetente contendo o status "commit." Caso contrário, será recebida com status "abort."

5) Um protocolo two phase commit, tal como o descrito por Birman [Bir], será usado para garantir a entrega atômica.

6) Somente membros podem receber mensagens de um grupo. Para fazê-lo, é usado um procedimento que tem como parâmetros o identificador de grupo, o endereço de uma área de memória para armazenar a mensagem (buffer) e o tamanho desta área. Um inteiro é devolvido indicando o tamanho da mensagem recebida. Se nenhuma mensagem estiver disponível, o processo remetente é bloqueado até que uma mensagem se torne disponível.

7) Haverá uma função para retornar a informação sobre um grupo, que estará disponível para qualquer processo. Esta função terá como parâmetros o identificador do grupo e o tipo de informação desejada e devolverá a informação em um buffer.

Um método de implementar este serviço é com um protocolo de três camadas sobre uma rede confiável como a descrita acima. A camada inferior consistirá de uma entidade para a comunicação de grupo confiável que se chama Group Protocol Entity (ou GPE), uma por máquina no sistema distribuído. O conjunto de GPEs proverá uma entrega atômica para todas as máquinas no sistema. A camada intermediária proverá a ordenação da mensagens e a camada superior proverá a capacidade de se ter grupos dinâmicos.

Uma vez que a segunda camada proverá a ordenação das mensagens, será necessário implementar apenas as operações Send e Receive. Quando o sistema é inicializado, a regra de ordenação terá de ser escolhida. Esta camada simplesmente recebe mensagens da camada acima e as envia para a rede, e recebe mensagens da rede e as envia para cima de acordo com uma dada regra de ordenação. Quando uma men- 
sagem é recebida de uma camada acima, essa camada adicionará um cabeçalho que inclui a informação necessária para a ordenação, passando-a para a GPE. Quando uma mensagem é recebida da rede, esta camada determinará se a mensagem pode ser entregue, dependendo das mensagens recebidas e a informação de ordem no cabeçalho da mensagem atual. Se a mensagem não pode ser entregue imediatamente, ela é armazenada até que todas as mensagens enviadas previamente tenham chegado. A regra de ordenação determinará o que significa o termo enviadas previamente.

O restante deste trabalho trata somente da camada mais inferior (a GPE) das três camadas. Futuros trabalhos podem tratar as demais. Uma vez que seja determinado um bom modo de especificar, implementar e testar a camada GPE, a especificação e teste das outras duas camadas não devem apresentar maiores problemas.

\subsection{Descrição do $G P E$}

Segue uma especificação informal de um serviço de broadcast atômico (GS) para um conjunto estático de processos de aplicações. O objetivo de tal serviço é fornecer uma abstração do envio atômico para um grupo de processos de aplicação. A interface entre a GPE e os processos de aplicação consiste dos dois canais de comunicação fromUser e toUser, os quais são usados para transmitir os dados referentes às mensagens e dados de controle.

As primitivas para utilizar estes canais são:

GPE_broadcast (message, members) é uma chamada não-bloqueante pela qual a disseminação de message é iniciada.

GPE_receive (message, members, from, result) é uma chamada que bloqueia o invocador e retorna somente quando toUser contém uma mensagem. Quando isso acontecer, os parâmetros são atualizados com os valores correspondentes a esta nova mensagem. O parâmetro result contem o estado final da mensagem, que pode ser committed ou aborted.

A funcionalidade básica do $G S$ é a seguinte: para cada pedido de disseminação, a GPE tentará entregar a mensagem para todos os membros. Se algum membro não estiver disponível, a disseminação é abortada e a mensagem não será entregue para nenhum membro. Neste caso, a disseminação é dita aborted. Caso todos os membros estejam disponíveis, então a mensagem é entregue a todos e a disseminação é dita committed.

O GS não garante qualquer tipo de ordenação na entrega das mensagens. Por exemplo, duas mensagens que são dependentes causalmente podem chegar em ordens diferentes em dois processos.

O GS implementa uma entrega de mensagens com semântica pelo menos uma vez. Isso significa que os processos de aplicação podem receber uma mensagem mais de uma vez. Isso não será um problema tratado pela $G P E$ pois é possível detectar e descartar as mensagens duplicadas em camadas superiores.

\subsubsection{Arquitetura}

O serviço GS será implementado por um conjunto estático de Entidades do Protocolo de Grupos (GPE), cada uma servindo um único processo de aplicação, como mostrado na figura 1. Para cada pedido de disseminação, a GPE ligada ao processo de aplicação que iniciou este pedido será chamada o coordenador do 


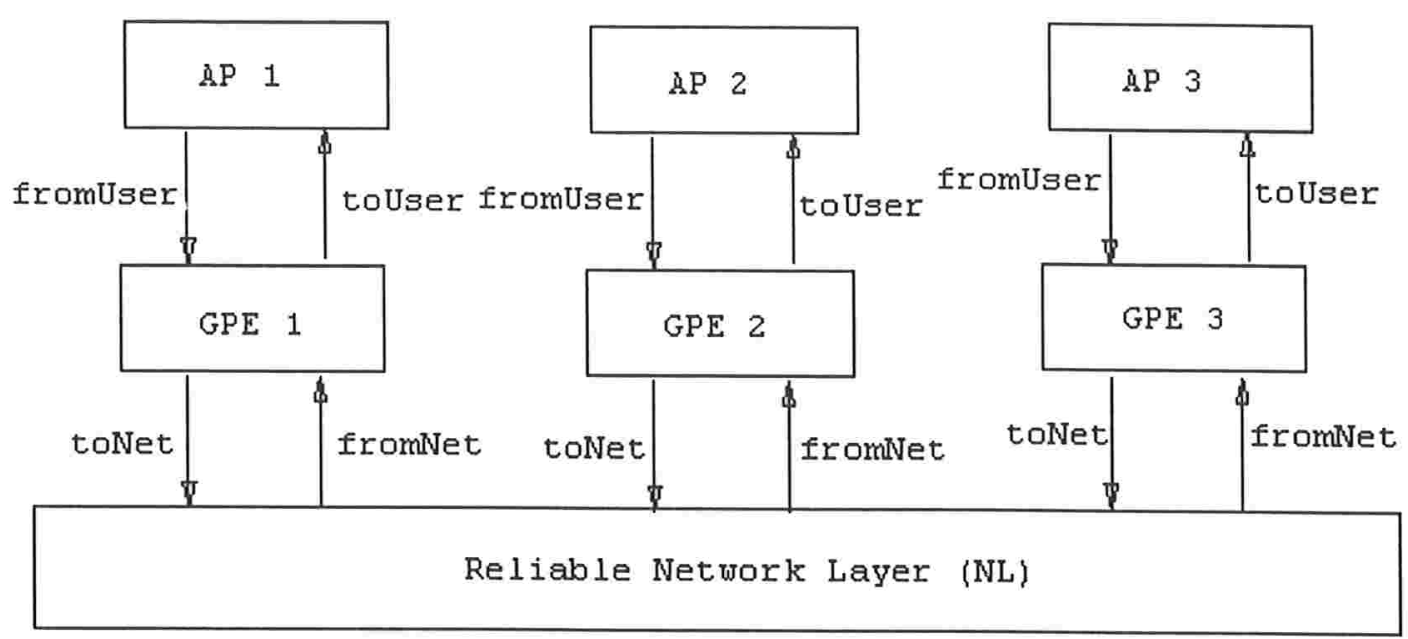

Figura 1: A Arquitetura do Serviço

pedido. O coordenador inicia o protocolo de confirmação de duas fases (two-phase commit) entre os GPEs para executar a disseminação atômica. O coordenador tentará entregar a mensagem para todos os GPEs, e se não for possível, não a entregará para nenhum deles.

GPEs enviam mensagens entre si usando os serviços de uma camada de rede confiável $(N L)$, que garante a entrega de mensagens mas não faz suposições sobre o tempo de transmissão das mensagens. A interface entre cada GPE e a camada de rede consiste dos dois canais de comunicação fromNet e toNet, os quais são usados para enviar mensagens pontuais (peer-to-peer) entre GPEs.

Associado a cada GPE deverá existir um dispositivo de armazenamento não-volátil que é usado para registrar o estado dos pedidos de disseminação. Isso permite que os GPEs que estão se recuperando de uma falha transiente possam retomar a entrega de mensagens de acordo com o seu estado imediatamente antes da falha.

\subsubsection{O Protocolo de Confirmação em Duas Fases}

O protocolo de confirmação em duas fases $(2 P C P)$, representado na figura 2 , pode ser explicado em duas situações:

\subsubsection{Processamento Normal}

Quando um pedido para disseminação é recebido no canal fromUser de um GPE (que assim torna-se o coordenador do pedido), este pedido é registrado como new e é disseminado a todos os outros GPEs.

Ao receber uma mensagem deste tipo, todos os GPEs ativos registram o pedido com o tipo OK. Durante um certo período, o coordenador coleta as respostas das demais GPEs. Tão logo todas as respostas tenham sido recebidas, o tipo da mensagem torna-se committed. Se o período termina e nem todas as respostas são recebidas, então o tipo da mensagem torna-se aborted. Este tipo é gravado, e o coordenador envia o resultado (committed ou aborted) a todos os GPEs e então entrega a mensagem ao processo que faz o pedido de disseminação. Na recepção de uma mensagem committed ou aborted, cada GPE também atualiza o tipo gravado. Se o tipo final é committed então a mensagem é entregue aos processo de aplicação. Caso contrário, é descartada. 


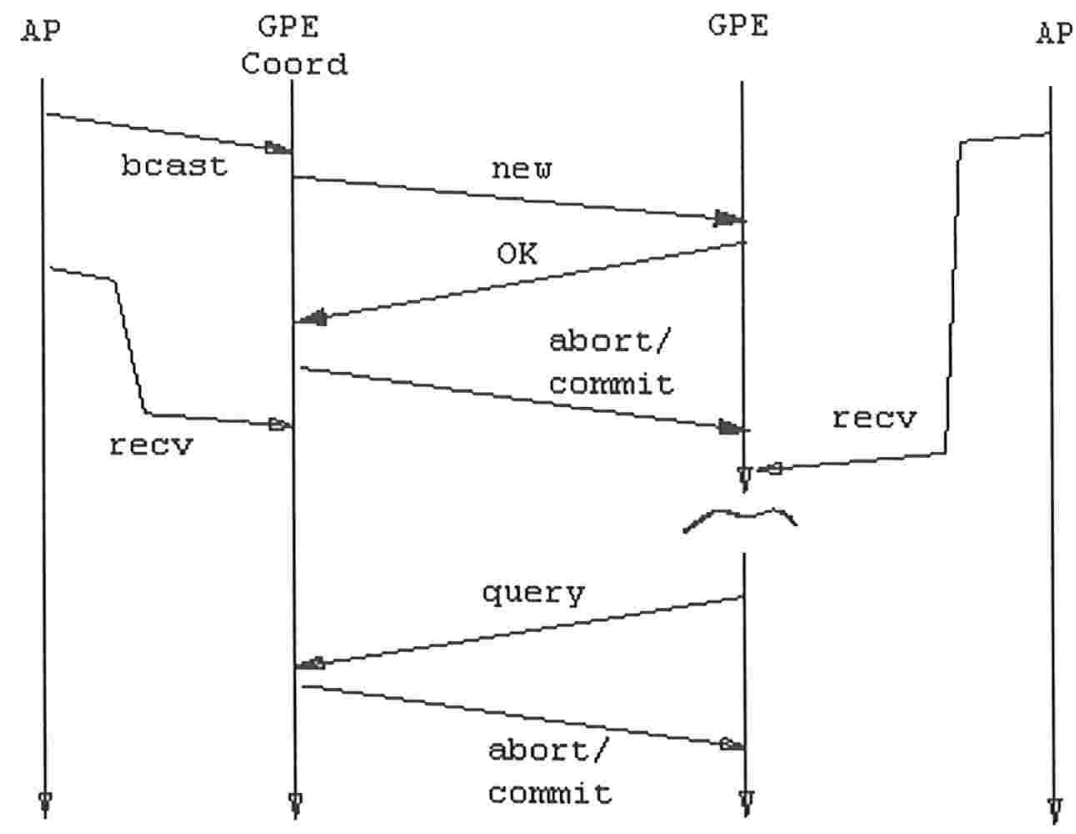

Figura 2: O Protocolo de Confirmação em Duas Fases

\subsubsection{Recuperação}

Quando uma GPE recomeça depois de uma falha, inicialmente esta analisa a lista de mensagens gravadas e faz o seguinte:

- Para cada mensagem com tipo new, é iniciado um novo $2 P C P$ para completar o pedido do qual foi o coordenador, mas que não havia sido completado. Todos as respostas recebidas antes da falha são descartadas.

- Para cada mensagem com estado OK, é enviada uma mensagem de tipo query para os demais GPEs para determinar o resultado do pedido. Já que cada GPE que sobreviveu a esta segunda fase do protocolo tem gravado o resultado do envio da mensagem, cada GPE ativa responde à query com o resultado.

A GPE deve retomar seu processamento normal enquanto está se recuperando.

\subsubsection{Falhas}

A qualquer momento um ou mais processos de aplicação e sua GPE podem tornar-se não-disponíveis durante um período arbitrário. Isso também vale para o coordenador de um pedido.

Uma vez que é suposto que a camada de rede seja confiável, nenhuma mensagem pode ser perdida pela rede. Isso significa que quando uma mensagem é enviada de uma GPE pelo canal toNet, esta mensagem eventualmente é entregue pela rede ao seu destino. Além disso, nenhuma mensagem pode ser corrompida pela rede. Consequientemente, a GPE só tem que tratar falhas do tipo parada.

Também é descartada a possibilidade de falha no meio de armazenamento não-volátil usado para armazenar as mensagens com o seu tipo. 
Além disto, assume-se que as seguintes ações serão atômicas:

a) receber um pedido do usuário e gravá-lo.

b) receber uma mensagem da rede e gravá-la.

Esta suposição de atomicidade é necessária para garantir a consistência entre os registros e as interações de GPEs com seus processos de aplicações.

\subsubsection{Suposições Adicionais}

- Cada GPE terá um endereço fixo chamado GPEaddr, pelo qual é conhecida pelas demais GPEs.

- Os registros que contém o estado dos pedidos podem ter um tamanho arbitrário.

- A demora para buscar e acessar os registros é insignificante.

- Os canais fromUser e toUser são capazes de guardar um número arbitrário de mensagens, que são consumidas na ordem FIFO.

\subsubsection{Propriedades Desejadas}

As seguintes são as principais propriedades desejadas do sistema:

Consistência Uniforme: Se uma mensagem chegar a um processo usuário, então eventualmente ela vai chegar a todos.

Terminação: Cada broadcast iniciado eventualmente chega ou ao estado commit ou ao abort.

Correção (1): Caso um broadcast torna-se abort, então a mensagem correspondente não chega em nenhum processo usuário.

Correção (2): Caso um broadcast torna-se commit, então a mensagem correspondente vai chegar a todos os processos usuários.

Garantia de Entrega: Se todos os GPEs são disponíveis entre o pedido de um broadcast e a entrega do seu resultado a um processo usuário, então a mensagem correspondente tem estado commit.

Ausência de Disseminação espontânea: Se uma mensagem for entregue a qualquer processo usuário, então tem que ter havido um pedido para o seu broadcast.

Capacidade de Recuperação: Cada processo que se recupera eventualmente recebe todas as mensagens que têm estado final committed.

\section{Linguagens de Especificação Formal}

Foram analisadas duas linguagens de especificação para protocolos de comunicação: LOTOS como descrita em [LOT], e Promela, como descrita em [Hol]. Há várias outras, tais como: Estelle, SDL etc.

As especificações em LOTOS descrevem processos que se comunicam enviando dados por meio de portas (gates) compartilhadas. Os processos têm como parâmetros os dados normais e as portas (sem especificação de tipo) que são usadas para se comunicar com outros processos. Os dados são representa- 
dos por especificações algébricas. Os processos têm condicionais e recursão como estruturas de controle, bem como mecanismos de sincronização e paralelismo. Uma semântica formal para LOTOS já foi definida. LOTOS com tipos de dados é chamada LOTOS Completa (Full LOTOS), enquanto que a linguagem sem tipos de dados é chamada LOTOS Básica (Basic LOTOS).

Promela é uma combinação de LOTOS e C. Ela inclui a maioria das características de LOTOS e outras, tais como um mecanismo para timeouts e para marcar um grupo de ações como atômico. Os únicos tipos de dados disponíveis são aqueles incluídos na linguagem (por exemplo, char, int e array). Tipos Abstratos de Dados (ADTs) não são disponíveis.

Um uso de semânticas formais que Gordon [Gor] apresenta é o de prover conceitos abstratos, precisos, independentes de uma máquina. A maior limitação da Promella é que ela não provê tais conceitos. Uma vez que os comportamentos de vários tipos de dados são definidos como sendo iguais aos dos tipos correspondentes de ANSI C, estes podem variar dependo da implementação. Sem uma semântica formal, é impossível fazer afirmações gerais precisas sobre o comportamento de uma especificação escrita em Promella.

Uma vez que LOTOS foi a linguagem escolhida, é interessante notar algumas das suas principais características. Sistemas distribuídos são descritos em LOTOS em termos de processos. Um sistema é um processo composto de outros processos que executam e interagem através de ações atômicas. Um processo é considerado uma caixa preta que reage com seu ambiente. As ações que acontecem dentro da caixa não são visíveis e não fazem parte do modelo. Tais ações são chamadas de ações internas. As ações observáveis descrevem comunicações síncronas entre dois processos.

Os nomes dos processos usualmente começam com letras maiúsculas e os de ações com letras minúsculas. A ação interna é representada por i. Em LOTOS Completa, as ações de entrada são denotadas com um ponto de interrogação e as de saída com um ponto de exclamação (por exemplo, $g$ !v e $h$ ?x, em que v é uma expressão de um valor e $\mathrm{x}$ é uma variável).

O processo mais simples de LOTOS é representado por stop, que não faz nenhuma ação. Os outros processos são compostos de combinações de ações, e há quatro tipos de combinações básicas: o prefixação de uma ação a um processo, a escolha entre dois processos, a ocorrência de interrupção e a combinação paralela. Esta última combinação tem três tipos de sincronização: nenhuma, parcial e completa.

A composição seqüencial de ações é denotada por ";", que é usada para prefixar uma ação a um processo. Por exemplo, considere o processo $a ; B$. A ação $a$ será oferecida ao ambiente e, se acontecer, o processo continuará $\operatorname{com} B$.

O operador de escolha não-determinística $\square$ introduz opções para sincronização, e a seleção pode ser influenciada pelo ambiente. Por exemplo: $B_{l} \square B_{2}$ é um processo que executa ou uma ação inicial de $B_{I}$ ou uma de $B_{2}$, dependendo do ambiente. Se o ambiente oferece uma sincronização com uma ação inicial de $B_{1}$ e não com uma de $B_{2}$ então $B_{1}$ será escolhida, e vice-versa. Se uma ação inicial de $B_{1}$ e uma de $B_{2}$ forem oferecidas, uma escolha não-determinística é feita. Se o ambiente não oferece uma de $B_{1}$ nem de $B_{2}$, o processo é bloqueado até que pelo menos uma das ações seja oferecida pelo ambiente. A ação interna $\mathbf{i}$ nunca é bloqueada porque ela não necessita de uma interação com o ambiente.

O processo $B_{1} \| B_{2}$ denota a composição independente de $B_{1}$ e $B_{2}$. Estes dois subprocessos não se comunicam entre si. O comportamento da composição é o de intercalação (interleaving) arbitrária das 
ações de $B_{1}$ e $B_{2}$. Isto é, que as ações de um subprocesso acontecem na ordem especificada em relação às outras do mesmo subprocesso, mas podem ter qualquer ocorrência relativa com eventos do outro subprocesso.

No outro extremo de sincronização está o operador $B_{l} \| B_{2}$ que requer uma sincronização de todos os pares de ações em $B_{1}$ e $B_{2}$. Entre os dois operadores anteriores, existe o I[]l, que denota uma sincronização mais geral: o processo composto só tem que estar sincronizado com as ações listadas na caixa. Por exemplo, $B_{l}|[a, b]| B_{2}$ descreve a composição em que $B_{1}$ e $B_{2}$ têm que se sincronizar através de $a$ e $b$.

O operador de interrupção (disabling) é usualmente usado para modelar uma falha no sistema. Com esta interpretação de $B_{1}\left[>B_{2}, B_{1}\right.$ descreve a operação normal e $B_{2}$ descreve o que acontece depois da ocorrência de um evento, como por exemplo uma falha. A semântica de $B_{1}$ [ $>B_{2}$ é que a qualquer ponto da execução de $B_{l}$, uma ação inicial de $B_{2}$ pode acontecer. Depois de entrar em $B_{2}$, o fluxo em $B_{l}$ é abandonado para sempre.

Além da descrição de processos, em LOTOS Completa também há uma parte da linguagem que descreve a especificação de tipos de dados em uma linguagem algébrica. São os valores expressos nesta linguagem algébrica que são usados nas ações de comunicação. Predicados booleanos formados por valores podem guardar as escolhas não-determinísticas do operador $\square$. 


\section{Ferramentas Disponíveis}

LOTOS tornou-se um padrão internacional em 1989, e muitas ferramentas têm sido desenvolvidas para facilitar seu uso. LITE [Can] é um conjunto de ferramentas que inicialmente pareceu promissor. Atualmente dispomos da versão 3.0, instalada na rede do IME. Este conjunto foi desenvolvido pelo consórcio LOTOSPHERE e posto à disposição do público em 1992. Há quatro grupos principais de ferramentas dentro de LITE. Com eles, uma especificação pode ser analisada dinâmica e estatisticamente, validada e ter os casos teste gerados para os tipos de dados.

As ferramentas para a análise estática permitem a verificação de uma especificação em relação à sintaxe e à semântica de uma especificação (ferramenta TOPO). Permitem tembém a verificação da completeza e da consistência de seus tipos de dados (ferramenta Cooper), além de apontar as dependências entre processos (ferramenta Xref). A ferramenta ILDI (Interactive LOTOS Data Interpreter) auxilia na reescrita de expressões algébricas para sua forma normal. Smile, a ferramenta de análise dinâmica, permite a animação e a simulação de uma especificação. Dentre as demais ferramentas para validação, Verif é a mais interessante, pois permite a demonstração automática de teoremas escritos como fórmulas em lógica temporal. Infelizmente, essa ferramenta trabalha somente com LOTOS Básica, isto é, sem tipos de dados. Pior ainda é que ela não está incluída na distribuição do LITE, mesmo tendo a sua descrição incluída na documentação de LITE.

Loft, LOgic for Functions and Testing, é uma ferramenta para manipular especificações na linguagem de especificação algébrica PLUSS. Ela fornece muitas das mesmas características fornecidas por Smile para $A D T$ s, tais como estreitamento (narrowing) e resolução: um objetivo é apresentado à ferramenta, que então procura por soluções. A diferença principal entre as duas, além das diferenças entre as suas linguagens alvos, é que Smile usa uma estratégia de busca em profundidade e Loft usa uma de busca iterativa em profundidade, que em muitos casos tem a aparência de uma busca em largura.

Os algoritmos mais interessantes usados nas ferramentas de verificação são resolução por reescrita (rewriting) e verificação de modelos (model checking). O primeiro é parecido com Prolog com equações e predicados em vez de apenas predicados, em que as equações permitem que termos iguais possam ser substituídos. Eles também diferem em seus propósitos: Prolog é usado para instanciar variáveis com o fim de satisfazer um objetivo, enquanto rewriting tenta somente reduzir um termo à sua forma normal. Não existe a noção de retrocesso (backtracking) em sistemas de reescrita, portanto só pode existir uma equação que pode ser aplicada a cada passo. Resolução não é usada para instanciar variáveis no termo sendo reescrito, mas para unificar subtermos.

Uma possível razão para a ausência de ferramentas para validar as especificações em LOTOS Completa é o método usado pelas ferramentas existentes, o de model checking. Com este método, a especificação é inicialmente convertida para um autômato de estados finitos e as propriedades são escritas como fórmulas em uma lógica temporal. O autômato é considerado como um modelo da lógica. Para verificar uma propriedade ele é usado para determinar a presença ou ausência de certas combinações de estados. Por exemplo, "Não há um caminho com uma transição $a$ que continua até uma transição $b$." ou "após uma transição $a$ todos os caminhos passam uma transição $b . "$ 
Na ausência de dados, a verificação de uma propriedade utiliza o model checking para demonstrar a presença ou ausência de certos estados. Uma vez que LOTOS tem recursão e interrupção, até uma especificação relativamente curta pode requerer um autômato de estados finitos grande para representá-la. Mesmo assim, sistemas com até $10^{20}$ estados, ou mais, podem ser checados exaustivamente, com novos métodos ad hoc que evitam a construção do modelo inteiro.

Com a introdução de tipos de dados, o problema torna-se muito mais difícil. Cada estado de um autômato que corresponde a uma versão da especificação em LOTOS Básica tem que ser substituído por um conjunto de estados, cada um com um elemento para cada atribuição possível, e isto para todas as variáveis ativas na especificação. Fantechi et al. [Fan] mencionam um model checker para LOTOS Completa, mas esta ferramenta ainda está sendo desenvolvida. 


\section{A Especificação Formal}

A especificação formal descreve o comportamento das GPEs no contexto específico descrito na seção 5.2. Este contexto consiste de um grupo de cinco GPEs, seus cinco usuários e uma rede confiável. Os usuários e a rede são abstrações que fornecem a funcionalidade mínima que as GPEs precisam. Cada GPE tem vários arquivos (Pending, Outcome e Query) para armazenar os estados de mensagens, e estes arquivos são acessíveis somente para a GPE que os controla. As GPEs podem falhar a qualquer momento.

Uma especificação LOTOS consiste de uma seção com especificações dos ADTs e uma com especificações dos processos.

\subsection{Os $A D T$ s}

A seção dos $A D T$ s começa com três tipos simples que são sugeridos pela especificação informal. Cada um destes tipos vem acompanhado de operações para testar sua igualdade e desigualdade:

- Kindofmsg os vários tipos de mensagens

- Voter os três possíveis tipos de votos (OK, NOK e NN)

- GPEAddr os possíveis endereços das cinco GPEs no sistema

Os dois tipos de recipientes (containers) usados na especificação são listas e dicionários (também conhecido como vetores associativos). Os containers especificados são genéricos, no sentido em que eles descrevem o armazenamento e a retirada de elementos de tipos formais quaisquer e só depois são instanciados com tipos atuais. As coleções sugeridas pela especificação informal e instanciadas na especificação são

- Votedictionary

- Messagedictionary

- MembershipList para os votos de cada GPE para uma dada mensagem para as mensagens

para os endereços das GPEs no sistema

Um dicionário é um vetor associativo genérico, isto é, um vetor que pode ter qualquer tipo como índice. Suas operações são resumidas abaixo.

- ED
- Mod, Fill
- Remove
- Retr
- Choose
- IsIn, IsNotIn,
IsEmpty
- Size
- Count
o dicionário vazio (Empty Dictionary) adicionam pares de índices e atributos remove um dado índice devolve o atributo associado com um dado índice devolve um índice

dão informação sobre a pertência de um índice devolve o número de pares de índice e atributo devolve o número de ocorrências de um dado atributo

As mensagens trocadas pelas GPEs têm mais informações do que as fornecidas pelos usuários. Elas também contém informações de controle, e sendo que o conjunto todo é descrito pelo tipo MessageStatus. Os atributos deste tipo são: 


$\begin{array}{ll}\text { - Data } & \text { a mensagem do usuário } \\ \text { - Seq } & \text { o número de sequiência desta mensagem } \\ \text { - } \text { - Toord } & \text { o endereço da } G P E \text { que é o coordenador desta mensagem } \\ \text { - From } & \text { o endereço da GPE que é o recipiente desta mensagem } \\ \text { - Kind } & \text { o endereço da GPE que é o remetente desta mensagem } \\ \text { - Acks } & \text { o tipo desta mensagem } \\ \text { o dicionário de votos para esta mensagem }\end{array}$

\subsection{Os Processos}

O comportamento do sistema é definido pela interação de usuários, GPEs e a rede. Cada um dos cinco usuários tem uma GPE que é usada para coordenar suas atividades de grupo. As GPEs se comunicam pelo envio de mensagens através da rede. Os processos usuários e o processo rede não modelam usuários e uma rede real, mas em vez disso, só fornecem o contexto definido informalmente em que as GPEs operam.

A GPE é responsável pela a implementação do protocolo de confirmação em duas fases. Para fazer isso, são usados vários arquivos, que são implementados através do ADT File. Estes arquivos são locais e particulares a cada GPE. Sua funcionalidade é simplesmente o armazenamento de um dicionário de Messagestatus, que é atualizado quando um novo dicionário é apresentado e que devolve a última versão armazenada quando isto for pedido. Os três arquivos são Pending, Outcome e Query:

- Pending mensagens cujos resultados ainda não são conhecidos

- Outcome os resultados obtidos para cada uma das mensagens (committed ou aborted)

- Query consultas feitas por uma GPE, para as quais ainda não recebem resposta

O processo GroupProtocolWithRecovery mostra que durante a execução do protocolo uma falha pode acontecer a qualquer momento, e que neste caso apenas as informações armazenadas nos arquivos ficam disponíveis depois de uma falha.

O processo GroupProtocol começa por ler os arquivos. Quando o sistema foi inicializado, os arquivos foram criados vazios. O restante do processo GroupProtocol tem duas partes principais. A primeira é o tratamento de uma mensagem. A segunda é o processamento das entradas no arquivo Query. Finalmente é feita uma chamada recursiva para processar mais mensagens.

Na primeira parte, o protocolo tem três opções: fazer uma ação interna (que não é visível ao ambiente), receber uma mensagem do usuário ou receber uma mensagem da rede. Se a mensagem veio do usuário, então a mensagem é um pedido para uma disseminação ao grupo. O protocolo requer que esta mensagem seja gravada no arquivo Pending antes que a disseminação comece. Como vista na especificação informal, assume-se a ausência de falhas entre a recepção da mensagem e a gravação no arquivo.

Se a mensagem é da rede, existem várias possibilidades, dependendo do tipo da mensagem. Os tipos possíveis são OK, NOK, New, Commit, Abort e Query. Quando a mensagem é do tipo New, ela é gravada no Pending e o voto OK é enviado ao coordenador. Quando a mensagem é do 
tipo OK ou NOK, este voto é armazenado em Pending e quando todas os votos estão disponíveis então o processo IfCompleteSendResult efetua a definição do resultado.

Note-se que quando o tipo é Commit ou Abort, os coordenadores Outcome, Pending e Query são atualizados correspondentemente. O Query também é atualizado no caso do Commit/Abort ser uma resposta a uma consulta.

Quando uma GPE se recupera de uma falha, o arquivo Pending tem que ser processado. Isso é feito em paralelo com a operação normal. Uma GPE que está se recuperando não está imune a uma falha, e na verdade pode falhar a qualquer momento.

O processamento do arquivo Pending é feito percorrendo-se todos os seus elementos. Apenas mensagens de tipo New, e OK são armazenados neste arquivo. Para mensagens de tipo New, os votos recebidos antes da falha são descartadas e o protocolo é reiniciado. Para as mensagens de tipo OK que não estejam em Outcome, as demais GPEs são consultadas para descobrir o resultado da mensagem.

A especificação formal da GPE e sua explicação detalhada são apresentadas nos Apêndices B e C, respectivamente. Para um melhor entendimento da especificação formal, recomendase a sua leitura dirigida pelos comentários no Apêndice $C$. 


\section{Validação da Especificação}

Produzir tal especificação é útil para se ter uma descrição do sistema em uma forma mais abstrata do que em código, porém igualmente precisa. No entanto, esta especificação é ainda mais útil se for possível mostrar que ela realmente modela o sistema como o imaginamos. A especificação é composta de duas partes distintas que precisam ser validadas: as definições de tipos de dados $(A D T \mathrm{~s})$ e as definições dos processos.

\subsection{A Validação dos $A D T$ s}

A validação das especificações algébricas é simples. Foi criada uma lista de propriedades que os $A D T$ s devem possuir, expressa como teoremas em LOTOS, para que a ferramenta Smile pudesse ser usada para a sua validação. Se todos os teoremas puderem ser provados a partir dos axiomas, todas as propriedades são válidas. Os primeiros três tipos definidos (veja página A-5, começando nas linhas 7, 28 e 50) são tão simples que sua validação é trivial. Para cada um desses tipos, Smile foi usada para fornecer os pares de valores que são iguais e aqueles pares que não são. Por exemplo, $\mathrm{x}$ :Vote $\mathrm{T}$ eq $\mathrm{y}:$ Vote $\mathrm{T}=$ true tem como solução

$\mathrm{x}=$ OK_ack, $\quad \mathrm{y}=$ OK_ack,

$\mathrm{x}=$ NOK_ack, $\mathrm{y}=$ NOK_ack $\mathrm{e}$

$\mathbf{x}=$ NN_ack, $\mathbf{y}=$ NN_ack.

De forma similar, a ferramenta encontrou como solução de $\mathrm{x}$ :Vote $\mathrm{T}$ ne $\mathrm{y}: \operatorname{Vote} \mathrm{T}=$ true as outras seis combinações.

Os tipos de propriedades que desejamos mostrar para os tipos de dados recipientes (containers) são apenas um pouco mais interessantes; são um pouco mais difíceis de criar e muito mais difíceis de provar. Como Arnold et al. mencionam em [Arn], não é bom derivar tais listas de propriedades a partir da especificação, uma vez que assim fazendo, assumiria-se a validade da especificação e, dessa forma, estaria-se assumindo o que se quer mostrar. Há duas maneiras principais através das quais as propriedades são formuladas. A primeira é imposta pelas características das ferramentas, que obrigam o uso de uma abordagem mais construtiva do que aquela que a lógica exige (veja Apêndice C, página A-15). Isso torna-se necessário para a demonstração de que as descrições declarativas das operações se mantêm, como por exemplo, que vale $\operatorname{IsIn}(\operatorname{Remove}(L, e), e)=$ false.

A outra maneira é aplicar os observadores aos pseudo-construtores. Os construtores da lista são NIL e Con, e portanto todas as operações são definidas em termos destes. Em vez de usar Con, deve-se pensar que os construtores da lista são NIL, Add e Remove. Desta forma, é interessante certificar-se de que os observadores se comportam corretamente com respeito a estas três operações. A lista de propriedades que foi originalmente escolhida para verificação é apresentada no Apêndice D. Por exemplo, a propriedade Add-3 afirma que se um elemento está em uma lista então a adição de qualquer elemento a essa lista não pode removê-lo, ou simplificando, que a adição à lista não pode remover qualquer elemento.

Smile foi a ferramenta originalmente escolhida para validar os $A D T$ s, porém identificou-se que a mesma não é confiável. Usando Smile para validar MembershipList, esta ferramenta indicou que não existe solução para o objetivo $\operatorname{Is} \operatorname{In}(\operatorname{Con}(1, \theta), e e)=$ false. Isto é, após a adição de qualquer elemento a uma lista de GPEs, qualquer outra GPE está também na lista. A ferramenta também mostrou que existem exatamente cinco soluções para o objetivo 
$\operatorname{IsIn}(\operatorname{Con}(1, e), e \theta)=$ true. Essas cinco soluções correspondem a $\mathbf{e}=\mathbf{e e}$, significando que apenas a última GPE adicionada à Membershiplist está na lista. Contactamos então o pessoal da Universidade de Twente que dá suporte a Smile, e eles confirmaram que este é um problema conhecido com o procedimento de resolução da ferramenta. Assim sendo, Smile não foi mais considerada adequada para uso com estruturas de dados.

A ferramenta Loft foi a segunda opção escolhida. A semântica de PLUSS, a linguagem usada pelo Loft, é muito parecida com a da linguagem da definição de tipos de dados de LOTOS. A principal diferença em nosso contexto é que LOTOS permite a definição de tipos genéricos, enquanto que Loft não permite. Isso foi contornado validando-se containers concretos com chaves (key) e atributos (attrib) de tipos que podem assumir exatamente cinco elementos cada um. A tradução da especificação dos containers para PLUSS encontra-se no Apêndice E.

Loft não pretende ser um demonstrador geral de teoremas, além de ser capaz de fornecer alguns tipos de demonstrações. Esta ferramenta foi desenvolvida para ajudar na geração de casos teste e dá apoio apenas para a reescrita e resolução (rewriting e resolution) de termos, não havendo possibilidade para provas por indução. Mesmo assim, a maioria das propriedades que foram inicialmente propostas foram mostradas verdadeiras na especificação. No Apêndice $F$ encontra-se a lista das propriedades que foram submetidas à validação por Loft e os respectivos resultados. Note que as propriedades foram provadas por absurdo, isto é que a negação de cada uma das propriedades desejadas não tem solução. Uma vez que Loft não é um demonstrador de teoremas de propósito geral, ao invés de provar as propriedades para todas as listas e dicionários, foi escolhida uma instância de cada tipo de container e foram demonstradas as propriedades para todos os containers daquele tipo e de tamanho limitado. O tamanho de um valor, denotado $\alpha$, é o número de construtores usados para escrevê-lo em sua forma normal. Na especificação do GPE, votedictionary só aparece com uma Membershiplist de até cinco chaves. Assim, um limite de tamanho dez na lista verificada foi considerado como sendo mais do que o suficiente. Uma vez que o dicionário é muito mais complexo do que a lista, seria muito mais difícil verificá-lo usando o mesmo limite. Por isto foi escolhido um limite de tamanho cinco para este $A D T$.

As propriedades no Apêndice $\mathrm{F}$ que não estão marcadas foram demonstradas. Aquelas marcadas com uma adaga $(\dagger)$ foram demonstradas para containers de tamanho até cinco, ao invés de dez. Nenhum resultado significativo foi obtido para aquelas marcadas com uma adaga dupla ( ). A estatística indicando o número de propriedades demonstradas se encontra na tabela seguinte.

\begin{tabular}{lccc} 
Container & $\begin{array}{c}\text { Demonstrada } \\
\text { para } \alpha=10\end{array}$ & $\begin{array}{c}\text { Demonstrada } \\
\text { para } \alpha=5\end{array}$ & $\begin{array}{c}\text { Não } \\
\text { demonstrada }\end{array}$ \\
\hline Lista & 16 & 5 & 3 \\
Dicionário & & 14 & 14
\end{tabular}

$\mathrm{O}$ fato de que muitas propriedades do dicionário não puderam ser demonstradas com rigor formal foi um ponto negativo. Se o tempo permitisse, ferramentas mais poderosas (como PVS de Stanford ou HOL de Oxford) poderiam ser usadas para demonstrar a validade ou não das mesmas. No entanto, o tempo necessário para aprender a usá-las foi considerado excessivo e impraticável 
dentro do escopo deste trabalho, já que não acrescentaria muito ao grau de confiança da especificação.

Embora a especificação da GPE use Messagedictionary, que tem outros cinco possíveis atributos, essa instância do dicionário não foi validada devido a sua similaridade com voteDictionary.

\subsection{A Validação dos Processos}

A idéia básica para a validação dos processos foi similar. Uma lista de propriedades foi desenvolvida em paralelo com a especificação. Pretendíamos provar que cada uma das propriedades é uma consequiência da especificação. Um grupo mínimo de propriedades (da especificação completa) a serem verificadas encontra-se listado na seção 6 da especificação informal (veja pagina A-4). Na realidade, encontrar ferramentas para demonstrar essas propriedades foi muito difícil. A única ferramenta encontrada na literatura é apenas brevemente mencionada na sessão de trabalhos futuros do artigo escrito por Fantechi et al. [Fan].

[Can] descreve Verif, o verificador de modelos do projeto LITE, que verifica fórmulas em lógica temporal para uma especificação escrita em LOTOS Básica. Infelizmente, essa ferramenta não é distribuída com LITE, e está apenas disponível aos grupos que a desenvolveram. Smile permite outro tipo de abordagem: de acordo com sua documentação, é possível usá-la para encontrar os caminhos de execução (traces) de uma especificação para uma dada ação de comunicação. Infelizmente, já não foi possível demonstrar que uma mensagem pode eventualmente ser enviada ao usuário. Provavelmente isto é uma conseqüência do mesmo erro identificado no procedimento de resolução para $A D T$ s mencionado anteriormente (veja seção 9.1).

Devido aos problemas encontrados na validação dos processos, esta etapa só foi completada mais tarde, quando já havia se iniciado a implementação e os testes. O método usado era simplesmente uma inspeção da especificação e um re-exame da sua explicação. Mesmo depois deste processo, encontrou-se um erro durante a implementação. Em ProcessQueries, e as linhas originais 601-602 foram alteradas de

> writePending! Mod( Pending, pk,

setAllAcks (pm, OtherMembers, NN_ack));

para

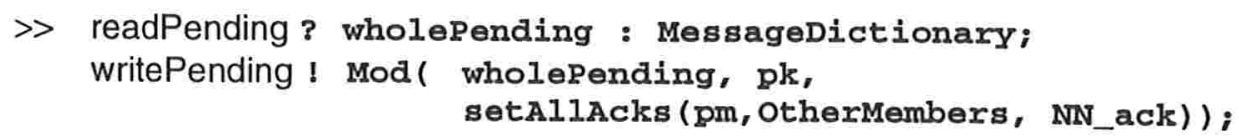

A justificativa para tal alteração é o seguinte: O processo usa a variável Pending que contém uma versão temporária do PendingDict, cujos elementos são retirados a longo da execução do processo. $\mathrm{Na}$ versão original, as modificações a esta cópia era escritos em arquivo, perdendo-se assim o conteudo original de PendingDict no arquivo.

Isto mostra como a tarefa de validação é importante para detectar propriedades indesejadas ou ausentes em uma especificação. 


\section{Criação de Casos Teste}

\subsection{Generalidades sobre Verificação}

\subsubsection{Técnicas de Verificação}

Quando uma especificação é transformada manualmente em um sistema executável por uma máquina, a conformidade da implementação com a especificação tem de ser verificada. Essencialmente, existem duas maneiras para fazê-lo: demonstrando estaticamente ou testando dinamicamente o sistema em execução. A técnica mais comum adotada para linguagens de programação procedural é o uso de triplos \{pré-condição; comando; pós-condição\} de FloydHoare, como apresentada originalmente em [Hoa].

A maior desvantagem deste método é que as provas são válidas apenas se a semântica da linguagem de implementação pode ser mapeada para aquela da linguagem de especificação. Uma vez que C não tem uma semântica formal, isto torna-se difícil, mas não impossível, haja visto que uma semântica formal tem sido definida para subconjuntos de C. No entanto, todos os comportamentos da linguagem que são dependentes da implementação ou estão indefinidos precisam também ser especificados. Algumas facilidades da linguagem têm de ser evitadas, como criar pseudônimos (aliasing) de áreas mutáveis de memória. É necessário demonstrar que a semântica formal da linguagem de programação se mantém para todas as combinações de compiladores e máquinas nos quais o código será executado. Uma outra característica desfavorável desse método é que o mesmo não pode ser usado para demonstrar que um sistema como um todo está correto, uma vez que não é possível especificar todos os aspectos da interação com o ambiente.

A demonstração estática de que uma função satisfaz as dadas pre- e pós-condições é difícil, mas fazê-lo dinamicamente (isto é, durante a execução) é simples. As pre-condições podem ser testadas (asserted) na entrada da função e as pós-condições testadas antes do retorno. Isso foi feito nas implementações dos $A D T \mathrm{~s}$.

\subsubsection{Teste de Conformidade}

Por causa da sua complexidade e de suas limitações, as técnicas de prova formal da correção de programas são utilizadas muito menos freqüentemente do que as técnicas de teste. O objetivo das técnicas de teste formal é derivar sistematicamente um conjunto de testes a partir da especificação, para uma dada noção formal de conformidade. Assim, aplicando-se com sucesso os testes na implementação, a conformidade da implementação com a especificação é garantida. Note-se que esse conjunto de testes deve ser derivado a partir da especificação e não a partir de uma implementação. A conformidade desejada para o protocolo é que todas e somente as mensagens especificadas (a serem envidadas para os Users e GPEs) serão realmente enviadas na implementação, ou seja, nenhuma mensagem pode ser perdida ou criada pelo sistema. Outros comportamentos (tais como a criação e uso de arquivos auxiliares) são aceitáveis. Brinksma [Bri] nota que o teste de robustez (robustness testing), isto é, testes da presença de tais comportamentos adicionais, não podem ser derivados metodicamente a partir da especificação, já que eles não estão descritos lá. 


\subsection{Geração de Casos Teste}

Uma vez que a estrutura da implementação foi derivada da estrutura da especificação, existem três partes principais que precisam ser testadas: o comportamento dos ADTs, o das ações e o dos processos, sendo que processos utilizam os dois anteriores. A derivação dos casos teste para cada uma das três partes é distinta. As ações são os componentes básicos em LOTOS e têm uma semântica elementar, mas a sua implementação geralmente requer mais de uma simples linha de código. Cada ação na implementação envolve um comportamento mais elaborado do que aquele definido pela semântica formal. Por esta razão, as ações foram testadas informalmente. A derivação dos testes para os $A D T$ s e para os processos é discutida na seção seguinte.

\subsubsection{Geração de Casos Teste para os $A D T$ s}

\subsubsection{Generalidades do método}

Testar código baseado em uma especificação algébrica consiste em demonstrar que cada um dos axiomas na especificação se mantém no sistema. Para criar casos teste para um dado axioma, as variáveis no axioma são substituídas por valores e a expressão booleana resultante é avaliada. Caso seu valor seja verdadeiro, então o teste é aprovado; senão ele é falho. Axiomas condicionais têm que ser manipulados com um pouco mais de cuidado: A premissa tem que ser verdadeira para o caso teste ser útil, uma vez que se há falsa, isto implicaria qualquer coisa.

Em [Gau], Gaudel faz uma discussão da base formal para testar um programa baseado em uma especificação algébrica. Tal especificação tem duas partes: uma assinatura (signature) $\Sigma=(S, F)$ na qual $S$ é um conjunto finito de tipos (sorts) e $F$ é um conjunto finito de nomes de operações sobre os sorts em $S$, e $A x$, um conjunto finito de axiomas que são $\Sigma$-fórmulas. Os axiomas são fórmulas condicionais positivas (possitive conditional formulas). Se SP é uma especificação e $\mathrm{P}$ é um programa sob teste, então o teste está relacionado aos valores computados por $\mathrm{P}$ que são mencionados em SP. O programa $\mathrm{P}$ tem que prover um método de executar as operações de modo que possa ser determinado se os axiomas de SP são satisfeitos. Seja $t$ um $\Sigma$-termo base (ground) e $t_{P}$ sua computação por $\mathrm{P}$. Dada uma $\Sigma$-equação $\varepsilon$ e um programa $\mathrm{P}$ que fornece uma implementação para cada operação de $\Sigma$, um teste para $\varepsilon$ é qualquer instância ground de $t=t^{\prime} \mathrm{em} \varepsilon$. Um experimento teste de $\mathrm{P}$ para $t=t^{\prime}$ consiste da avaliação de $t_{P}$ e $t_{P}{ }^{\prime}$ e a comparação dos valores resultantes. Essa comparação é a tarefa de um oráculo, isto é, de um procedimento que pode decidir se o resultado computado está correto.

Um conjunto exaustivo de testes para uma especificação é o conjunto de todas as instâncias de tipo correto e ground de todos os $\Sigma$-axiomas. Quando um programa passa o conjunto exaustivo $_{S P}$ isto não necessariamente significa que ele satisfaz SP, uma vez que exaustivo $_{S P}$ é exaustivo em relação a SP, mas não necessariamente a P. Portanto, P satisfaz SP somente se todos os valores computados por $\mathrm{P}$ podem ser alcançados por $\mathrm{T}_{\Sigma}$. Dado uma assinatura $\Sigma$, a hipótese mínima $H_{m i n}$ é aquela na qual P define uma $\Sigma$-álgebra gerada finitamente, e sendo portanto $\Sigma$ testável.

Freqüentemente exaustivo $S P$ é grande demais para ser útil. É possível propor hipóteses mais rígidas sobre o comportamento do programa e reduzir o número de testes necessários para demonstrar a satisfação da especificação. Essas são chamadas de hipóteses de seleção, e as mais comuns são hipóteses de uniformidade e de regularidade. Uma hipótese de uniformidade é a suposição de que o espaço de entrada pode ser dividido em sub-domínios tais que se um conjunto 
de teste que contém um único representante de cada sub-domínio e cada um destes elementos o teste é bem sucedido, então o teste para todos os elementos de exaustivo ${ }_{S P}$ também é bem sucedido. Um exemplo para inteiros é "se o sistema funciona para entradas de um valor negativo, um positivo e zero, então, funcionará para todos os inteiros." Uma hipótese de regularidade utiliza uma função de termos ground para os números naturais e tem a forma "se um conjunto de testes formado por todos os termos ground com tamanho menor que um dado limite é bem sucedido, então exaustivo $_{S P}$ também é." Um exemplo de tal hipótese para uma lista seria "se a operação Add funciona para todas as listas de tamanho de até quatro, então funcionará para todas as listas."

A estratégia de teste é definida pela escolha das hipóteses de seleção. Expor as hipóteses torna clara as suposições feitas no programa. Um contexto de teste é um par $(H, T)$ de um conjunto de hipóteses e um de testes. Um contexto de teste é considerado válido se $H$ implica que se $T$ é bem sucedido então exaustivo $S P$ é também. O contexto é considerado imparcial (unbiased) se $H$ implica que se exaustivo ${ }_{S P}$ é bem sucedido então $T$ é também. Assumindo $H$, a validade assegura que todos os programas são rejeitados, e imparcialidade assegura que nenhum programa correto é rejeitado.

Por construção, $\left(H_{\min }\right.$, exaustivo $\left.{ }_{S P}\right)$ é tanto válido quanto imparcial. Várias hipóteses podem ser formuladas simultaneamente sobre uma implementação. Um outro exemplo extremo válido e imparcial é $\left(H_{\min } \wedge \mathrm{P}\right.$ passa exaustivo exP,$\left.\varnothing\right)$, isto é, se é assumido que o programa está correto então não é preciso nenhum teste. Contextos de teste interessantes são aqueles que são válidos e imparciais, isto é, aqueles que são bem sucedidos por somente e todos os programas corretos. Hipóteses fracas correspondem a conjuntos grandes de testes, e hipóteses fortes a conjuntos pequenos. O objetivo é fazer hipóteses razoáveis para reduzir o conjunto de testes para um tamanho tratável. A seleção de tais hipóteses pode ser baseada na especificação formal, em algum conhecimento do programa ou em algumas das características do ambiente do sistema.

\subsubsection{Aplicação do Método}

Um conjunto é parcial se o mesmo rejeita algumas implementações corretas. Para evitar parcialidade no conjunto de testes, foram gerados casos teste das versões mais declarativas da especificação, conforme descrito na explicação da especificação (Apêndice C).

Sorts genéricos não podem ser testados diretamente, mas somente nas suas instanciações. O tipo de lista mais usado na especificação é MemberhipList, uma lista de GPEaddrs. Uma vez que a especificação considera apenas cinco GPEs, parece razoável adotar-se a hipótese de regularidade de tamanho cinco nas listas. Uma vez que existem apenas

$$
\sum_{i=0}^{5} P(5, i)=\sum_{i=0}^{5} \frac{5 !}{(5-i) !}=326
$$

permutações com tamanho menor ou igual a cinco, é possível determinar os valores para todas as combinações de operações, listas e GPEs. O número de experimentos de teste para esta estratégia está resumido na tabela seguinte: 


\begin{tabular}{|c|c|c|c|}
\hline Operação & $\begin{array}{c}\text { Combinações } \\
\text { de Listas }\end{array}$ & $\begin{array}{c}\text { Combinações de } \\
\text { Outros Parâmetros }\end{array}$ & $\begin{array}{c}\text { Número de } \\
\text { experimentos }\end{array}$ \\
\hline$\overline{\text { Add }}$ & 326 & 5 & 1630 \\
\hline Remove & 326 & 5 & 1630 \\
\hline IsIn & 326 & 5 & 1630 \\
\hline IsNotin & 326 & 5 & 1630 \\
\hline IsEmpty & 326 & 0 & 326 \\
\hline IsNotmmpty & 326 & 0 & 326 \\
\hline Choose & 326 & 0 & 326 \\
\hline Length & 326 & 0 & 326 \\
\hline Total & & & $\overline{7.824}$ \\
\hline
\end{tabular}

O menor dos dois dicionários usados na especificação é o voteDictionary, que usa GPEaddr como chave e voteT como atributo. O número de votedictionarys possíveis é muito maior do que o número de MembershipLists. São

$$
\sum_{i=0}^{5} P(5, i) \cdot 3^{i}=\sum_{i=0}^{5} \frac{5 !}{(5-i) !} \cdot 3^{i}=40.696
$$

dicionários com tamanho menor ou igual a cinco. Uma das operações, fill, toma como parâmetros um VoteDictionary, uma lista de GPEaddr, e um voteT. Um teste exaustivo dessa operação, até com a hipótese de regularidade mencionada acima, iria requerer $40.696 \cdot 326 \cdot 3=$ 39.800.688 experimentos. Usando-se a hipótese de uniformidade tanto em GPEaddr como voteT, o número de dicionários que tem que ser testados se reduz para seis. Testando fill com todas as 326 listas iria requerer então 1956 combinações. Fazendo uma hipótese de uniformidade nos GPEaddrs das Membershiplists diminui o número de experimentos para trinta e seis, menos que um milionésimo do número original. Os valores armazenados nas seis listas e nos seis dicionários podem ser gerados aleatoriamente desde que seja produzido um exemplo de cada tamanho possível de zero a cinco.

Nenhum dos axiomas que define fill é condicional, portanto foi possível utilizar quaisquer dicionários com GPEaddrs e voteTs aleatórios. Isso também é válido para Choose, IsEmpty e size. Para as demais operações (isto é, Remove, IsIn e Count) as hipóteses são razoáveis somente se as premissas dos axiomas são verdadeiras (uma vez que falso implica qualquer coisa). Para evitar a geração de valores individuais para cada um destes testes condicionais, a hipótese de uniformidade foi omitida nos GPEaddrs destas operações, e todos os cinco valores foram testados. O número de experimentos necessários para esta estratégia está mostrado na tabela seguinte:

\begin{tabular}{|c|c|c|c|}
\hline Operação & $\begin{array}{l}\text { Dicionários } \\
\text { e Listas }\end{array}$ & $\begin{array}{l}\text { Outros } \\
\text { Valores } \\
\end{array}$ & $\begin{array}{c}\text { Número de } \\
\text { Experimentos }\end{array}$ \\
\hline Mod & 6 & 5 & 30 \\
\hline Remove & 6 & 5 & 30 \\
\hline Fill & 36 & 1 & 36 \\
\hline Retr & 6 & 5 & 30 \\
\hline IsIn & 6 & 5 & 30 \\
\hline IsNotIn & 6 & 5 & 30 \\
\hline IsEmpty & 6 & 0 & 6 \\
\hline IsNotmpty & 6 & 0 & 6 \\
\hline Choose & 6 & 0 & 6 \\
\hline Size & 6 & 0 & 6 \\
\hline Count & 6 & 3 & 18 \\
\hline Total & & & 228 \\
\hline
\end{tabular}




\subsubsection{Criação de Testes para os Processos}

O processo mais relevante na especificação é GroupProtocol. A geração de casos teste para este processo é muito diferente do que aquela para os outros processos por causa das suas naturezas. Manna e Pnueli [Man] descrevem estas naturezas como características transformacional e reativa. Uma função transformacional produz um resultado após uma computação finita. Um processo reativo, por outro lado, "não produz um resultado final mas mantém uma relação contínua com seu ambiente." GroupProtocol é reativo. Uma vez que os demais processos são transformacionais, a geração de casos teste para eles é similar àquela para os $A D T \mathrm{~s}$, onde o modo pelo qual suas entradas estão relacionadas às suas saídas é definido na especificação. GroupProtocol não trata de tal mapeamento, mas ao invés disso descreve os fluxos de informação através de iterações infinitas.

Durante o teste dos processos, foi assumido sempre que as ações atômicos e outros processos funcionam corretamente.

\subsubsection{Geração de Testes para Processos Transformacionais}

O processo mais simples é Broadcast (Apêndice B, linha 616), que tem dois parâmetros, Msgstatus e MembershipList. Este processo envia a mensagem para todos os GPEaddrs na MembershipList. Uma vez que a variável Msgstatus não é modificada ou testada, parece natural formar uma hipótese de uniformidade para ela. Uma hipótese de regularidade de tamanho cinco é feita para Membershiplist. Então Broadcast é testado com seis grupos de entrada, cada um com uma MembershipList de tamanho variando de zero a cinco.

O segundo processo testado foi ProcessPending (Apêndice B, linha 574), que é usado no processo Recovery. Como o nome sugere, seu principal parâmetro é PendingDictionary, e mais especificamente, o campo kind de seus atributos. O teste é dividido em duas etapas: a primeira mostrando que cada tipo de mensagem é manipulado corretamente e a segunda que PendingDictionarys de tamanhos diferentes também o são. A primeira parte é executada pela cobertura de cada uma das afirmações condicionais. As hipóteses feitas são de regularidade de tamanho um (1) no PendingDictionary, e de uniformidade para todos os campos da mensagem, que é o único atributo do dicionário, exceto para o campo kind. Existem dois tipos de mensagem que podem estar em um PendingDictionary: New e ok. Se uma mensagem é New, existem dois casos a serem considerados: Ou a mensagem está no outcomeDictionary ou não está. Uma vez que Msgstatus foi testada individualmente, as hipóteses feitas na segunda parte são: uma de uniformidade nos atributos do Msgstatus do outcomeDictionary, e uma de regularidade de tamanho cinco na Membershiplist. As saídas previstas estão resumidas na tabela seguinte.

\begin{tabular}{|c|c|c|c|}
\hline kind & $\begin{array}{l}\text { Mensagem no } \\
\text { outcomeDictionary }\end{array}$ & $\begin{array}{l}\text { Mudança no } \\
\text { PendingDictionary }\end{array}$ & $\begin{array}{l}\text { kind no } \\
\text { broadcast }\end{array}$ \\
\hline New & $\overline{\operatorname{sim}}$ & removido & do outcomedictionary \\
\hline New & não & todos os votos para NN & New \\
\hline OK & - & nenhuma & Query \\
\hline
\end{tabular}

ProcessQueries é um processo simples que é similar ao Broadcast, exceto que ao invés de fazer um broadcast de uma mensagem só, este faz um broadcast de um dicionário de mensagens. O QueryDictionary tem somente mensagens do tipo oK. É feita uma hipótese de uniformidade 
nos atributos do Msgstatus do dicionário e uma hipótese de regularidade no dicionário de tamanho cinco. As saídas previstas são aquelas nas quais cada uma das mensagens no QueryDictionary é broadcast com tipo Query, e o QueryDictionary não é modificado.

O último processo finito a ser testado é IfCompleteSendResult (linha 503), que é chamado depois do recebimento de cada confirmação (acknowledgement). Seu único parâmetro de interesse é um Msgstatus. O votedictionary, que contém as confirmações de uma mensagem msg pelos GPEs, é devolvido pela função GetAcks (msg). Se todos as GPEs no votedictionary da mensagem tiverem respondido, então essa mensagem é confirmada (committed).

Uma vez que não há confirmação negativa (negative acknowledgements) na implementação, (veja a Justificativa, página A-19), não há como testar a mesma em relação ao comportamento especificado.

De acordo com a especificação, há sempre quatro GPEs na lista de destino. Uma hipótese de uniformidade é feita para todos os campos da mensagem. Dois casos são considerados: Um no qual todas as confirmações são ok e um outro no qual nem todas são. No segundo caso, nenhuma saída é esperada. Já no primeiro caso, as saídas previstas são chamadas para a porta toUser e a execução de Broadcast a mensagem com kind alterada para Commit, e a inclusão desta mensagem modificada no OutcomeDictionary, e remoção correspondente do PendingDictionary.

\subsubsection{Generalidades sobre a Geração de Testes para Processos Reativos}

Como mencionado anteriormente, derivar casos teste para o processo GroupProtocol apresenta muitas dificuldades. Enquanto a noção de uma implementação correta de um $A D T$, ou de um processo transformacional, é baseada em satisfação lógica, aquela de um processo reativo é baseada em inclusão (containment) de comportamentos.

Uma estrutura matemática bem conhecida no estudo da semântica de processos é a teoria de Sistemas de Transição Marcada (Labeled Transition Systems, ou LTS). A semântica da linguagem de especificação LOTOS tem sido descrita em termos de sistemas de transição. LTS são variantes de autômatos, usando a noção de estados. Ações são modeladas como transições, e a ação interna é rotulada com $\tau$. O LTS do processo $\mathbf{Q}=a ; \mathbf{i} ; b ; \mathbf{Q}$ é mostrado na seguinte figura, onde Q0, Q1 e Q2 representam os possíveis estados de Q.

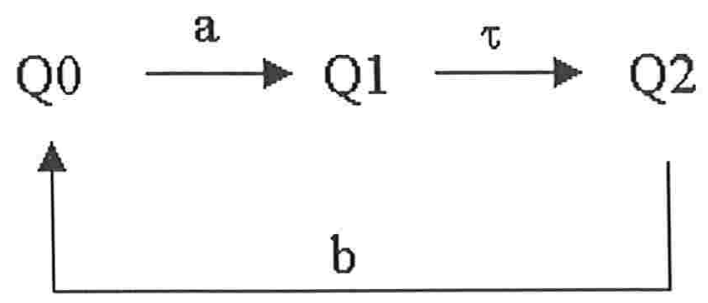

O teste de $A D T$ s depende da demonstração da equivalência de valores computados e previstos. Definir a equivalência de processos é mais complicado devido à possibilidade de paralisação completa (deadlocks) e do não-determinismo, no caso geral. Apenas as ações e os comportamentos observáveis são de interesse para o teste. Desta forma as relações de equivalência desenvolvidas deveriam considerar apenas estas ações. Uma relação de equivalência prospectiva é 
a de caminhos (trace equivalence). Um trace é uma seqüência de ações observáveis. A sequiência vazia é denotada por $\varepsilon$. Como exemplo, o conjunto de traces para $\mathbf{P}=a ; \mathbf{i} ; b ; a$ é $\{\varepsilon, a, a b, a b a\}$. Dois processos são considerados como equivalentes por caminhos (trace equivalent) se seus conjuntos de traces são iguais. Essa relação seria uma candidata para a definição de uma implementação correta de um processo por um outro (isto é, para ser a relação de conformance). Contudo, a pré-ordem de trace, definida pela inclusão de conjuntos de traces pode ser uma escolha melhor em alguns casos, uma vez que uma implementação correta tem que, ao menos, permitir comportamentos requisitados pela especificação mas pode permitir alguns outros.

A noção de traces observáveis não capta todas as observações que podem ser feitas em um processo pois um processo é executado em algum contexto, que consiste de outros processos que interagem com ele. Com este tipo de observação é possível identificar os processos que são trace equivalent. Por exemplo, considere os processos $\mathbf{S}=a ; b$ e $\mathbf{I}=\mathbf{i} ; a ; b \square a$. Se o processo $\mathbf{S}$ é uma especificação, como podemos determinar se o processo I é uma implementação correcta? Tem-se que Traces $(\mathbf{I}) \subseteq \operatorname{Traces}(\mathbf{S})$, mas em alguns contextos I tem comportamento diferente de $\mathbf{S}$. Por exemplo, Sll $a ; b$ sempre termina com sucesso enquanto Ill $a ; b$ pode, ou terminar com sucesso, ou alcançar um estado em que ele recusa $b$ e entra em deadlock.

Processos usualmente são testados desta maneira, sendo executados em paralelo com alguns outros processos chamados de testadores. Uma outra definição de equivalência interessante, chamada de testing equivalence, é a seguinte: dois processos $\mathbf{P}$ e $\mathbf{Q}$ são equivalentes se, para todo processo testador $\mathbf{T}$, suas composições com $\mathbf{T}$ são equivalente de algum modo, isto é, $\mathbf{P} \mathrm{t}_{\mathrm{eq}} \mathbf{Q} \leftrightarrow(\forall \mathbf{T} \mathbf{P} \| \mathbf{T}$ eq $\mathbf{Q} \| \mathbf{T})$. Existem várias testing equivalences dependendo da relação de equivalência escolhida para a composição.

Brinksma [Bri] trata da noção de deadlock destas composições paralelas. Em tal abordagem, a noção de conjuntos de recusa (refusal sets), ou a noção complementar de conjuntos de aceitação (acceptance sets) é essencial. A composição paralela com qualquer testador tem que ter as mesmas em falhas (ou seja failure equivalent), isto é, eles têm que ter os mesmos deadlocks.

No LTS associado com um processo $\mathbf{P}$, dado um estado $s$, denota-se out(s) o conjunto de ações observáveis de um trace iniciado a partir de $s$. Diz-se que $s$ é estável se não há uma transição rotulada por $\tau$ a partir de $s$. Dado um conjunto $A$ de ações observáveis, $s$ ref $A$ se $A \cap$ out $(s)$ $=\varnothing$. Isto é, no estado $s$, as ações em $A$ não podem ser executadas e portanto são recusadas. Dada uma seqüência $\sigma$, seja $\mathbf{P}$ after $\sigma$ o conjunto de estados que podem ser alcançados a partir do estado inicial de $\mathbf{P}$ depois de executar $\sigma$ (podem existir vários por causa de não-determinismo). Para cada $\mathbf{P}$ after $\sigma$ estamos interesados nos conjuntos de ações recusadas pelos estados de $\mathbf{P}$ after $\sigma$. Ref então é definida como este conjunto de conjuntos de ações:

$$
\operatorname{Ref}(\sigma, \mathbf{P})=\{A \subseteq L \mid \exists s \in \mathbf{P} \text { after } \sigma, \text { tal que } s \text { ref } A\}
$$

em que $L$ representa o conjunto de todas as ações observáveis. $A \in \operatorname{Ref}(\sigma, P)$ expressa que é possível mas não necessário que $\mathbf{P}$ bloqueie para todas ações em $A$ depois da execução de $\sigma$. Note que $\varnothing$ é um elemento de $\operatorname{Ref}(\sigma, \mathrm{P})$ sempre que $\mathrm{P}$ after $\sigma$ não é vazio. 
Por exemplo, considere o seguinte processo I e o diagrama LTS correspondente:

$$
\begin{array}{lll}
\mathbf{I}=\mathbf{i} ; a ; b \square a & \mathbf{I} 3 \in \mathbf{I} \text { after } a & \mathbf{I 1} \in \mathbf{I} \text { after } a \\
\operatorname{out}(\mathbf{I})=\{a\} & \text { out }(\mathbf{I} 3)=\{b\} & \text { out }(\mathbf{I} 1)=\varnothing \\
& \mathbf{I} \text { ref }\{a\} & \mathbf{I} \operatorname{ref}\{a, b\} \\
\operatorname{Ref}(\varepsilon, \mathbf{I})=\{\varnothing,\{b\}\} & & \operatorname{Ref}(a, \mathbf{I})=\{\varnothing,\{a\},\{a, b\}\}
\end{array}
$$

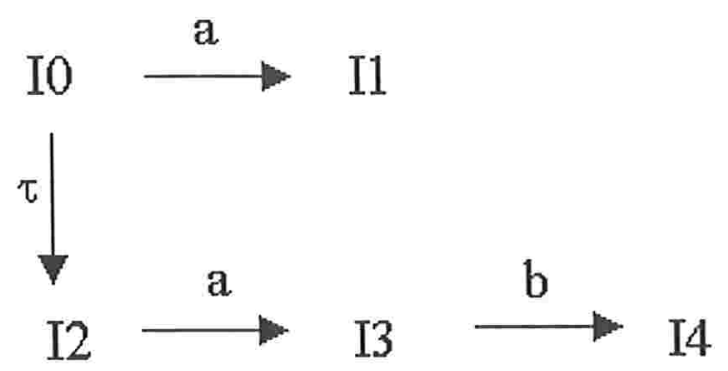

Conjuntos de recusa não são usados diretamente na derivação de testes. No lugar destes conjuntos, é usada a noção complementar de conjuntos de aceitação, ou seja, os conjuntos de transições que podem sair de um estado. Isto leva ao seguinte conjunto de conjuntos de ações:

$$
\operatorname{Accept}(\sigma, \mathrm{P})=\{A \subseteq L \mid \forall s \in \mathbf{P} \text { after } \sigma, \neg(s \operatorname{ref} A)\}
$$

$A \in \operatorname{Accept}(\sigma, \mathbf{P})$ expressa que depois da execução da sequiência de ações $\sigma, \mathbf{P}$ é sempre capaz de executar pelo menos uma das ações no conjunto $\boldsymbol{A}$. Os conjuntos de ações em $\operatorname{Accept}(\sigma, \mathbf{P})$ são chamados conjuntos de aceitação (acceptance sets) de $\mathbf{P}$ after $\sigma$. Hennessy [Hen] nota que $L$, o conjunto de todas as ações observáveis é um elemento de $\operatorname{Accept}(\sigma, \mathbf{P})$ sempre que $\mathbf{P}$ after $\sigma$ não é vazio.

Conjuntos de aceitação são utilizados para derivar testes, mas nem todos deles são úteis: Apenas aqueles que estão contidos em $\boldsymbol{o u t}(\mathbf{P})$ podem ser alcançados a partir de $\mathbf{P}$. Isto leva a definição de conjuntos obrigatórios (must sets):

$$
\boldsymbol{M}_{\varepsilon}(\mathbf{P})=\{\operatorname{out}(\mathbf{P}) \backslash A \mid A \subseteq \operatorname{Ref}(\varepsilon, \mathbf{P})\}:
$$

A relação de conformidade considerada por Brinksma [Bri] é definida como

$$
\text { I conf } \mathrm{S} \text { iff }(\forall \sigma \in \text { traces }(\mathrm{S}))(\operatorname{Ref}(\sigma, \mathrm{I}) \subseteq \operatorname{Ref}(\sigma, \mathrm{S}))
$$

Isto é, para todos os traces parciais $\sigma$ de $\mathbf{S}$ e todos os subconjuntos $A$ de ações observáveis, se $\mathbf{I}$ contém um estado after $\sigma$ que recusa $\boldsymbol{A}$, então existe tal estado em $\mathbf{S}$. Cada uma das recusas da implementação estão especificadas. Uma definição equivalente por Tretsman [Tre] é

$$
\text { I conf S iff }(\forall \sigma \in \operatorname{traces}(\mathrm{S}))(\operatorname{Accept}(\sigma, \mathrm{S}) \subseteq \operatorname{Accept}(\sigma, \mathrm{I}))
$$

Brinksma [Bri] descreve uma derivação de testes baseada nesta relação de conformidade e define uma transformação de um LTS para um outro LTS, chamado de testador canônico, que está definida para o caso de LTSs com um conjunto finito de ações observáveis e com traces finitos. Isto se aplica a um subconjunto de LOTOS Básica sem recursão. O testador canônica é a composição não-determinística de vários casos teste que são consistentes (sound). Existe apoximadamente um caso teste para cada trace completo no LTS original, com a possibilidade da introdução de ações internas em todo lugar. A execução paralela de uma implementação candidato com o testador produz um veredicto positivo quando o trace termina com sucesso, e um negativo se acontecer um deadlock. Por exemplo, considere o processo especificado $\mathbf{S}=b ; c \square \mathbf{i} ; a$, cujo tes- 
tador canônico é $C T(\mathbf{S})=\mathbf{i} ; b ; \mathbf{i} ; c ; \mathbf{i} \square \mathbf{i} ; a ; \mathbf{i}$. Executando $\mathbf{I} \| C T(\mathbf{S})$ para algum $\mathbf{I}$, o resultado é bem sucedido quando $C T(\mathbf{S})$ termina e mal sucido caso contrário.

O testador canônico é intrinsicamente não-determinístico. Por construção, as escolhas dos vários casos teste começam com uma ação interna, e portanto, não podem ser influenciadas pelo comportamento da implementação. Isto é altamente desejado, mas levanta a questão da cobertura (coverage) dos casos teste. Todos os casos teste podem ser executados através da execução do testador por várias vezes, mas isto não pode ser garantido. O testador canônico tem a vantagem de descrever um conjunto de testes em uma notação compacta. Contudo, uma apresentação mais realística seria um conjunto de testes determinísticos a serem executados independentemente.

Tretsman [Tre] descreve uma generalização do testador canônico para LTSs com comportamentos infinitos (mas ainda com um conjunto finito de ações observáveis). O problema principal é manter os casos testes finitos. Isto está feito pela parada a cada transição com um veredicto de sucesso. Este método produz um conjunto infinito de casos testes finitos. O conjunto completo de testes é então composto por todos os casos testes que são finitos, mais arbitrariamente compridos. Isso é parecido com a noção de conjunto exaustivo de testes dado por Bernot et al. em [Ber]. A escolher de um conjunto finito de casos teste geralmente corresponde à suposição de algum tipo de hipótese de regularidade na implementação.

A generalização para um conjunto infinito de ações observáveis é possível somente para os LTSs que têm imagem finita. Em um processo com imagem finita, o número de estados pode ser infinito, mas o número de estados que podem ser alcançados para um dado trace $\sigma$ é finito: isto é, $\forall \sigma \in \operatorname{traces}(\mathbf{P}), \mathbf{P}$ after $\sigma$ é finito. O tipo de linguagens formais que pode ser tratado com estas limitações se assemelha a LOTOS Completa, na qual existem um conjunto infinito de ações de entrada da forma $g$ :?x:tipo. Tretsman [Tre] analisa a derivação de testes para $B E X_{v}$, uma linguagem pequena definida atrevés seguintes processos:

stop nenhuma ação posterior é tomada

$g$ ?x:p; $B$ se $p$ então $x$ assuma o valor apresentado na porta $g$ e continue $\operatorname{com} B$ senão stop

$\mathrm{g} ! \mathrm{x} ; \mathrm{B} \quad$ apresente o valor de $x$ na porta $g$ e continue $\operatorname{com} B$

i: $p ; B \quad$ se $p$ então $B$ senão stop

$B_{1} \square B_{2}$ escolha não-deterministicamente entre $\mathrm{B}_{1}$ e $\mathrm{B}_{2}$

Por exemplo, a expressão $\mathbf{B}=g$ ? $\mathrm{x}: \mathrm{x}<20 ; h ! \mathrm{x}+2 ; \mathbf{s t o p} \square \mathrm{i} ; g$ ? $\mathrm{y}: \mathrm{y}>10 ; h$ !y;stop apresenta um comportamento válido. Tretsman elabora uma maneira de derivar casos testes, mas não há mais o conceito de um testador canônico, porque ele não pode ser expresso na linguagem $B E X_{v}$ uma vez que ele não é do tipo imagem finita.

A derivação de casos teste é simples e recursiva. Primeiramente, uma ação inicial do processo é escolhida em um dos conjuntos obrigatórios (must sets). Posteriormente, o resto do teste é derivado dos comportamentos dos processos testados após esta ação. Para agilizar a derivação, Tretsman define as seguintes funções: 


\begin{tabular}{|c|c|c|c|c|}
\hline$\underline{\mathbf{B}}$ & out $(\mathrm{B})$ & st (B) & $\boldsymbol{M}_{\varepsilon}(\mathrm{B})$ & \\
\hline stop & $\varnothing$ & true & $\{\varnothing\}$ & \\
\hline$g ? x: p ; B_{1}$ & $\{g ? \mathrm{x}: \mathrm{p}\}$ & true & $\{\{g ? \mathrm{x}: \mathrm{p}\}\}$ & \\
\hline i: $p ; B_{1}$ & $\begin{array}{ll}\text { out }\left(\mathrm{B}_{1}\right) & \text { se } \mathrm{p} \\
\varnothing & \text { se não-p }\end{array}$ & not $\mathrm{p}$ & $\begin{array}{l}A_{\varepsilon}\left(B_{1}\right) \\
\{\varnothing\}\end{array}$ & $\begin{array}{l}\text { se p } \\
\text { se não-p }\end{array}$ \\
\hline$B_{1} \square B_{2}$ & $\operatorname{out}\left(\mathrm{B}_{1}\right) \cup \operatorname{out}\left(\mathrm{B}_{2}\right)$ & $\begin{array}{l}\text { st }\left(\mathrm{B}_{1}\right) \text { and } \\
\text { st }\left(\mathrm{B}_{2}\right)\end{array}$ & $\{\operatorname{out}(\mathrm{B})\}$ & $\begin{array}{l}\cup \text { se não-st }\left(\mathrm{B}_{1}\right) \text { entao } \boldsymbol{M}_{\varepsilon}\left(\mathrm{B}_{1}\right) \\
\cup \text { se não-st }\left(\mathrm{B}_{2}\right) \text { então } \boldsymbol{M}_{\varepsilon}\left(\mathrm{B}_{2}\right)\end{array}$ \\
\hline
\end{tabular}

\begin{tabular}{|c|c|c|}
\hline B & choice B after $h ! v$ & \\
\hline stop & stop & \\
\hline$g ? x: p ; B_{1}$ & $\begin{array}{l}\mathrm{i} ; B_{1}[\mathrm{v} / \mathrm{x}] \\
\text { stop }\end{array}$ & $\begin{array}{l}\text { se } g=h \text { e } \mathrm{p}[\mathrm{v} / \mathrm{x}] \\
\text { se } g \neq h \text { ou não-p[v/x] }\end{array}$ \\
\hline $\mathbf{i}: p ; B_{1}$ & $\begin{array}{l}\text { choice } B_{1} \text { after } h ! \mathrm{v} \\
\text { stop }\end{array}$ & $\begin{array}{l}\text { se } p \\
\text { se não-p }\end{array}$ \\
\hline$B_{1} \square B_{2}$ & choice $B_{1}$ after $h ! \mathrm{v}$ & choice $B_{2}$ after $h$ !v \\
\hline
\end{tabular}

Um teste de $\mathbf{B}$ então é definido como ( $h_{1} ! \mathrm{v}_{1}$; test(choice $\mathbf{B}$ after $\left.h_{1} ! \mathrm{v}_{1}\right)$

\section{$\square h_{2} ! \mathrm{v}_{2}$; test (choice B after $h_{2} ! \mathrm{v}_{2}$ )}

$\ldots h_{\mathrm{n}} ! \mathrm{v}_{\mathrm{n}}$ \}é um elemento de $\boldsymbol{M}_{\varepsilon}(\mathbf{B})$.

$\ldots \square h_{\mathrm{n}} ! v_{\mathrm{n}}$; test(choice B after $\left.\left.h_{\mathrm{n}} ! \mathrm{v}_{\mathrm{n}}\right)\right)$, em que $\left\{h_{1} ! \mathrm{v}_{1}, h_{2} ! \mathrm{v}_{2}\right.$,

Do exemplo acima, out $(\mathbf{B})=\{g ? \mathrm{x}: \mathrm{x}<20, \mathrm{~g}$ ? $\mathrm{y}: \mathrm{y}>10\}, \quad \mathbf{B}$ não é estável $\mathrm{e}$ $M_{\varepsilon}(\mathrm{B})=\{\{g ? \mathrm{x}: \mathrm{x}<20, g ? \mathrm{y}: \mathrm{y}>10\},\{g ? \mathrm{y}: \mathrm{y}>10\}\}$. Um caso teste é obtido pela escolha de um conjunto em $M_{\varepsilon}(\mathrm{B})$, cujas variáveis são instanciadas com valores concretos, e a continuação da derivação para o resto do processo. Por exemplo:

- Escolhe $\{g$ ? $\mathrm{y}: \mathrm{y}>10\}$.

- Instancie com $g ! 11$.

- Um teste é $g$ ! 11, seguido da obtenção do teste de (choice B after $g$ !11).

Continuando o exemplo, o teste derivado será $g ! 11$; um_teste_para $\left(\mathbf{B}_{1}\right)$, em que $\mathbf{B}_{1}=\left(\mathbf{i} ; h\right.$ ?y:y=11;stop $\square \mathbf{i} ; h$ ?y:y=13;stop). A derivação para $\mathbf{B}_{1}$ segue o mesmo padrão com $\operatorname{out}\left(\mathbf{B}_{1}\right)=\{h ! 11, h ! 13\}, \mathbf{B}_{1}$ não é estável, e portanto $\mathbb{A}_{\mathcal{E}}\left(\mathbf{B}_{1}\right)=\{\{h ! 11, h ! 13\},\{h ! 11\},\{h ! 13\}\}$. Como no primeiro passo as ações são

- Escolhe $\{h ! 11, h ! 13\}$.

- Não havendo variáveis livres as ações têm que ser $h ! 11$ e $h$ !13.

- Um teste é $h$ !11; test(choice $\mathbf{B}_{1}$ after $h$ !11) $\square h$ !13; test(choice $\mathbf{B}_{1}$ after $h$ !13). Isto é, $h$ ! 11 ; test(i; stop) $\square h ! 13$; test(i; stop) e finalmente $h ! 11$; stop $\square h ! 13$; stop.

Então um teste $\mathbf{T}$ do processo original B é $g ! 11 ;(h ! 11$; stop $\square h ! 13$; stop). Se a execução do processo B\|T termina, então o teste é dito bem sucedido, senão é mal sucedido. 


\subsubsection{Aplicação ao GroupProtocol}

Para traduzir GroupProtocol para BEX, é conveniente notar que qualquer comunicação com os arquivos são ações internas para GroupProtocol, e qualquer seqüência de ações internas é equivalente a uma só ação interna. Assim, a versão simplificada de GroupProtocol em BEX passa a ser:

1. $G P=($ i: true;

2. $\square \quad \square$ fromUser ? msg : true; i:true; Broadcast (msg)

3. $\square$ fromNet ? msg : kind(msg)=oK $\wedge$ is InPending (msg); IfCompleteSendResult $(\mathrm{msg})$

4. $\quad \square$ fromNet ? msg : kind(msg)=oK $\wedge$ isNot InPending(msg)

5. $\square$ fromNet ? msg : kind(msg)=New; i:true; toNet ! $f(\mathrm{msg})$

6. $\quad \square$ fromNet? msg : $\operatorname{kind}(\mathrm{msg})=$ Commit $\wedge$ isInPending $(\mathrm{msg})$; $\mathbf{i}$ :true; toUser ! msg; i:true

7. $\square$ fromNet ? msg : kind(msg)=Commit $\wedge$ is InNot Pending(msg)

8. $\square$ fromNet ? msg : $\operatorname{kind}(\mathrm{msg})=$ Abort $\wedge$ isInPending $(\mathrm{msg})$; i:true;

9. $\square$ fromNet ? msg : kind(msg)=Abort $\wedge$ is InNotPending $(\mathrm{msg})$

10. $\square$ fromNet ? msg : $\operatorname{kind}(\mathrm{msg})=$ Query $\wedge$ isInPending(msg); toNet ! $g(\mathrm{msg})$;

11. $\square$ fromNet ? msg : kind(msg)=Query $\wedge$ isInNotPending $(\mathrm{msg})$

12. $) ;($ ProcessQueries

13. $\square \mathrm{i}$ :true

14. );GP

em que $f$ e $g$ são funções que alteram o tipo da mensagem.

O método de Tretsman foi modificado levemente para tornar os casos teste determinísticos e terminantes. A primeira modificação foi motivada pelo comentário na pagina 21 sobre o testador canônico, isto é, queremos uma cobertura de todos os casos. A segunda é brevemente sugerida por Tretsman e consiste em limitar os testes aos comportamentos finitos.

Então, há um teste por linha da especificação simplificada. Estas correspondem aos inícios dos testadores dados por Tretsman. Note que a linha 1 abaixo representa o teste de um processo que contém apenas ações internas. Usando o método descrito por Tretsman com as modificações mencionadas acima, os testadores para as linhas 1 a 11 são

1. $\varnothing$

2. fromUser ! msg: true; test(Broadcast $(\mathrm{msg})$ )

3. fromNet ! msg : $\operatorname{kind}(\mathrm{msg})=\mathrm{OK} \wedge$ is InPending $(\mathrm{msg})$; test(IfCompleteSendResult $(\mathrm{msg})$ )

4. fromNet ! msg: kind $(\mathrm{msg})=0 \mathrm{~K} \wedge$ isNot InPending $(\mathrm{msg})$

5. fromNet ! msg : kind (msg)=New; toNet ? $f(\mathrm{msg})$

6. fromNet ! msg : kind(msg)=Commit $\wedge$ is InPending(msg); toUser ? msg

7. fromNet ! msg : kind $(\mathrm{msg})=$ Commit $\wedge$ is InNotPending $(\mathrm{msg})$

8. fromNet ! msg : kind (msg)=Abort $\wedge$ isInPending $(\mathrm{msg})$

9. fromNet ! msg : kind (msg)=Abort $\wedge$ isInNotPending $(\mathrm{msg})$

10. fromNet ! msg : kind(msg)=Query $\wedge$ isInPending $(\mathrm{msg})$; toNet ? $g(\mathrm{msg})$;

11. fromNet ! msg : $\operatorname{kind}(\mathrm{msg})=$ Query $\wedge$ isInNotPending $(\mathrm{msg})$

Uma vez que os processos Broadcast, IfCompleteSendResult e ProcessQueries têm sido testados por outros meios, não foi necessário produzir os seus testadores. O próximo passo na geração dos casos teste foi produzir uma mensagem para cada uma das linhas 2 a 11 acima e enviá- 
las para a implementação sendo testada. Uma hipótese de regularidade foi formulada sobre todos os campos das mensagens enviadas, exceto o campo kind. As iterações de GroupCommunication dependem do conteúdo dos seus arquivos de dicionários. Se for possível mostrar que cada uma das execuções têm uma seqüência de ações de comunicação correta e deixa os dicionários no estado consistente, então a GPE deve funcionar para qualquer combinação de entradas. Isso é parecido com uma hipótese de tamanho um formulada para um $A D T$. 


\section{A Implementação}

Apesar de haver uma especificação formal antes da implementação, a implementação de algumas partes foi mais difícil do que inicialmente previsto. Entre estas partes está a comunicação entre processos através de portas e o armazenamento de informação em uma forma que evita a sua corrupção em caso de falha. A solução usada para a primeira está apresentada na seção seguinte, enquanto que aquela para a segunda encontra-se nas seção 11.2.2. Em particular, decisões e considerações de implementação tratam dos aspectos de eficiência e facilidade de uso e limitações de recursos do sistema, assuntos que não fazem parte da especificação.

Outros detalhes importantes da implementação não são mencionados nem na especificação formal nem na informal. Entre estes estão

- o hardware em que o sistema deve executar

- a linguagem em que ele deve ser escrito

- o compilador a ser usado

- o tipo de rede a ser usado

Estes eram conhecidos desde o início do projeto, mas não foram especificados formalmente. $\mathrm{O}$ sistema deveria rodar na rede do IME, que consiste de estações de trabalho Sun rodando Solaris. A linguagem de programação escolhida foi $\mathrm{C}$ e o ambiente de desenvolvimento o sistema Concert/C.

Concert/C foi escolhido porque é uma extensão de ANSI C que dá apoio à programação distribuída. Este apoio é realizado através da encapsulação da complexidade envolvida com a criação de processos e a comunicação entre os mesmos em primitivas da linguagem. No ambiente Concert/C, os processos comunicam-se entre si usando ou a troca assíncrona de mensagens ou a chamada remota de procedimentos (remote procedure call, RPC). Tais comunicações acontecem através de uma ligação (binding) de um processo com uma fila de mensagens de um outro processo. Tanto para $R P C$ como para a comunicação assíncrona, o compilador Concert/C verifica a compatibilidade entre os tipos argumentos/parâmetros e das mensagens. Todos os tipos de dados de $\mathrm{C}$ podem ser transmitidos entre processos, até aqueles complexos envolvendo apontadores. Estruturas de dados com apontadores são marcadas pelo programador para fornecer informações adicionais ao compilador para determinar como elas devem ser tratadas.

A comunicação assíncrona é feita usando as primitivas send e receive. As $R P C$ s são transparentes ao programador usuário no sentido que ele só tem que fornecer um binding para a função e o sistema de run-time empacota e transmite os dados automaticamente. Quando um processo chama uma função não local, a função realmente chamada é um procedimento stub, criado pelo compilador e que redireciona os dados para o processo que contém a função desejada usando as primitivas send e receive. Ao receber os dados, o servidor executa a função e os resultados são devolvidos para o stub no processo inicial. Finalmente, o stub devolve os resultados para a função que o chamou como se fosse ela que os calculou.

Em Concert/C há várias maneiras para interligar os processos. Quando um processo cria um processo filho, o pai recebe uma porta inicial que pode ser usada uma primeira comunicação com o filho, por exemplo para estabelecer bindings com outros portes do mesmo. Bindings podem ser transmitidos através da comunicação entre processos como quaisquer outros dados ou podem ser armazenados em arquivos compartilhados pelos programas. 
O compilador do Concert/C é um pré-processador que executa depois do pré-processador ANSI. As extensões da linguagem são substituídas por código ANSI C que é então passado ao compilador. As definições de portas são traduzidas para definições de estruturas. As chamadas para funções não locais têm seus stubs criados e também os outros comandos Concert/C são transformados em chamadas às funções de uma biblioteca run-time. Na documentação é mencionado que as partes de um programa que não fazem parte das extensões fornecidas são passados ao compilador sem modificações. Na realidade, elas podem sofrer mudanças que alteram sua semântica, causando problemas como descrito em seção 11.1.

\subsection{A Arquitetura do Sistema}

Como descrito na especificação, foi implementado um sistema que consiste de processos de usuários e GPEs sendo executados em cima de uma camada de rede confiável, que é fornecida pelo ambiente Concert/C. Cada máquina host contém um par de GPE e usuário. A especificação não menciona nem como os processos GPE e usuário devem ser criados nem como eles são inicialmente ligados entre si. GPEs são instanciadas pelo programa launcher, que tem que ser executado antes de qualquer programa de usuário que deseja usar o serviço de broadcast. A sequiência de inicialização está resumida no diagrama seguinte.

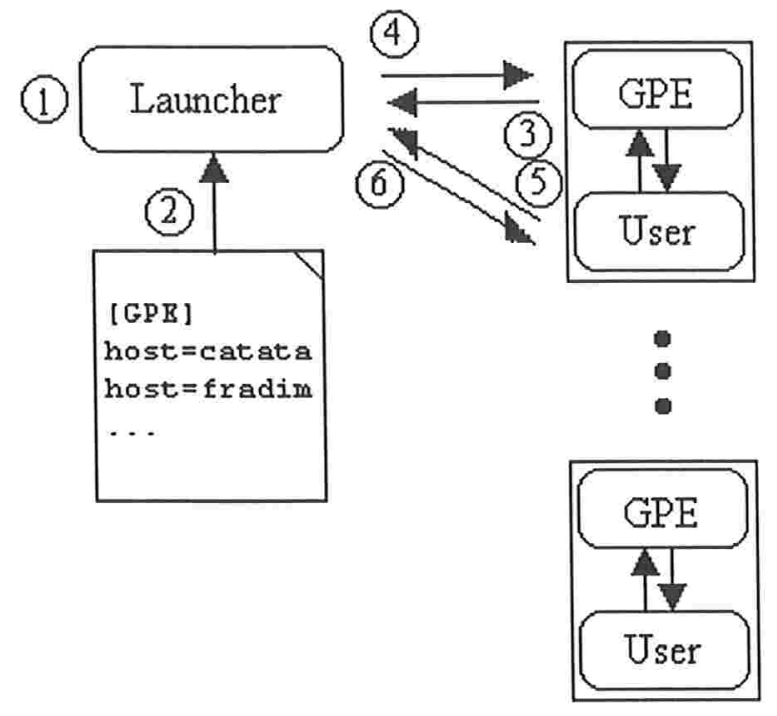

O código fonte para o launcher.c encontra-se no Apêndice M.

Um arquivo com nome GCs. ini (para Group Communication Service) descreve a configuração inicial do sistema. Este arquivo pode ser usado por todas as camadas do serviço de comunicação de grupo, e não apenas pela camada de GPEs. Um exemplo do arquivo está listado embaixo. Após o cabeçalho de seção [GPE], os valores de diversos time-outs são seguidos pela lista de máquinas host.

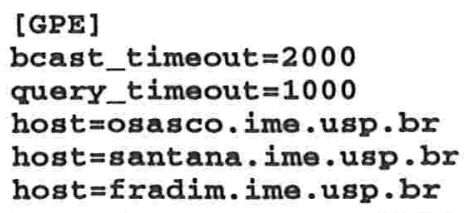

O launcher lê este arquivo e cria um processo GPE em cada uma das máquinas listadas. Cada um destas GPEs é consultada para descobrir o endereço da sua porta GPEtoGPE. Depois da recep- 
ção destas $n$ portas, $n^{2}$ mensagens são enviadas para informar as GPEs dos endereços das demais GPEs.

Após esta interação com o launcher, cada GPE é capaz de receber mensagens da rede e executar o protocolo sem esperar até seu usuário registrar. Se uma mensagem chegar a uma GPE antes do registro do usuário correspondente, esta mensagem é armazenada em uma fila que pode ser recuperada caso haja uma falha no sistema. Assim que um processo de usuário se registrar, todas as mensagens nesta fila são enviadas e a fila é destruída.

Para um processo de usuário ligar-se a uma $G P E$, inicialmente este deve obter ao launcher os endereços das portas daquela GPE. Após da recepção desta informação, ele manda uma mensagem que o anuncia à GPE. Com isso feito, um usuário pode iniciar o envio de broadcasts ao grupo. A interface de usuário para o sistema no nível das GPEs consiste de apenas duas funções, GPE_broadcast para o envio e GPE_receive para o recebimento de mensagens.

bool GPE_broadcast (char * sMsg, unsigned uLength,

GPEaddr_list *members) ;

unsigned GPE_receive(char * sMsg, GPEaddr * from,

GPEaddr_list * members, KindofMsgT * result) ;

Encontra-se no Apêndice G uma descrição detalhada desta interface.

Dado que o sistema foi projetado para ser robusto, uma GPE que falha pode ser substituída por outra através da execução do comando gpe recover sGPEdir na máquina desejada, em que sGPEdir é o nome completo do caminho do diretório que contém o arquivo Gcs.ini. Esta GPE que está se recuperando entra em contato com o launcher para informá-lo o endereço de suas portas e obter as portas dos demais GPEs. Usuários também podem ser substituídos a qualquer momento pelo registro de um novo usuário com a GPE desejada. Note que isto é uma potencial falha na segurança do sistema, uma vez que o usuário antigo não é consultado antes de ser descartado.

O objetivo principal de todas as técnicas da engenharia de software é a produção de sistemas corretos. O segundo é produzir tais sistemas de uma maneira que eles sejam fáceis tanto de entender como manter. O primeiro objetivo é tratado pelos métodos formais usados, mas o segundo não é. A especificação e a justificativa fornecem uma boa descrição abstrata do sistema, e o código reflete tanto quanto possível a estrutura da especificação. A especificação é composta de três tipos de construções: as definições dos $A D T$ s, as ações atômicas de comunicação e os processos. A abordagem usada na implementação de cada uma destas partes foi diferente, e o código para cada uma foi isolado.

\subsection{Os $A D T s$}

O propósito da seção dos $A D T$ s é a definição dos tipos a serem usados e as funções que os manipulam. Cada um dos tipos especificados tem duas partes: uma assinatura que fornece tanto os nomes dos tipos como também informações dos tipos para as operações naqueles tipos e axiomas que descrevem o comportamento das operações. Em C, as assinaturas são usualmente colocadas em arquivos de cabeçalho e os axiomas encontra-se em arquivos ".c". 


\subsubsection{Containers Simples}

Os tipos de containers, as listas e dicionários, são especificados como tipos genéricos que podem ser instanciados para armazenar quaisquer tipos que fornecem uma relação de igualdade. ANSI C não fornece uma maneira de realizar isso facilmente. Em C++, os containers seriam implementados usando o mecanismo de templates e sobrecarregando o operator $==($ ). Uma versão estática de templates simples pode ser simulada em $\mathrm{C}$ usando substituição de macros com parâmetros. Veja Appendix H para uma parte do arquivo list.h. Cada um dos tipos genéricos tem duas partes: uma declaração e uma definição. Cada uma dessas está implementada com um macro que é parametrizado pelo nome do tipo a ser armazenado. Este nome do tipo é usado para a criação das estruturas e funções necessárias na realização da instanciação. Já que LOTOS e C++ apoiam a sobrecarrega de nomes de funções, os nomes das funções que manipulam instaciações distintas podem ser os mesmos (por exemplo, nil pode se referir tanto a uma lista de GPEaddrs como também a uma lista de MessageIDs). Em C++ é feita através da manipulação (mangling) pelo compilador das nomes das funções (isto é, pela adição ao nome de uma função de informação sobre o número e os tipos dos seus parâmetros). Os macros dos container fazem algo semelhante (por exemplo, a instanciação de uma lista de GPEaddr e uma outra de MessageID produzirá (entre outras coisas) as funções nil_GPEaddr_list e nil_MessageID_list). A maior diferença entre os dois métodos é que mangling está feita na hora da compilação e somente o lincador tem acesso aos nomes que são mangled. Já que a substituição de macros é feita pelo preprocessador, o compilador está apresentado com seus efeitos e o programador tem que ser consciente dos nomes sendo gerados.

\subsubsection{Containers Estáveis}

Como foi mencionado anteriormente, do ponto de vista da especificação, os dicionários não podem falhar porque é assumido que eles são estáveis. No mundo real não é tão simples assim, pois estruturas de dados armazenadas na memória, como aquelas descritas acima, são perdidas quando acontece uma falha em um processo. Arquivos em disco são mais estáveis do que arquivos na memória, e o $A D T$ file, que é modelado como uma seqüência de registros que podem ser acessados por índice, foi criado para fornecer uma maneira de armazenar informações em disco. Uma estrutura de dados pode ser armazenada em um file e lida ou excluída dele. Um dado é armazenado usando a operação add: O valor é escrito em um lugar vazio do file e seu índice é devolvido. Para recuperar ou excluir um registro, precisa-se de seu índice. O número de registros armazenados em um file pode ser determinado, e além disto um file pode ser destruído inteiramente.

Guardar cópias da informação em disco é um passo no sentido certo, mas sempre há a possibilidade de uma falha (de processo ou de máquina) no meio do acesso de um arquivo, o que poderia corromper o seu conteúdo. Para resolver este problema, uma segunda cópia do arquivo pode ser feita, e com um arquivo indicador de qual dos dois arquivos garantidamente está não corrompido. Esta é a idéia atrás do $A D T$ st_file, que usa duas instâncias do tipo file chamadas $a$ e $b$ e um arquivo auxiliar e temporário. Sua interface para o resto do sistema é idêntica ao do file com a exceção de que o mesmo não pode ser corrompido por causa da falha de um processo. Quando um arquivo é modificado, os seguintes quatro passos são feitos:

- o arquivo $a$ é modificado

- o arquivo indicador é criado

- o arquivo $b$ é modificado

- o arquivo indicador é excluído 
Quando um st_file está sendo aberto, se existe o arquivo indicador, então sabe-se que o arquivo $a$ está não corrompido. Caso contrário, sabe-se que o arquivo $b$ é o não corrompido. Em qualquer caso, o arquivo incorrupto é copiado em cima daquele possivelmente corrupto e o indicador é excluído. Os observadores devolvem valores associados com o arquivo simples $a$.

Um dicionário estável, st_dict, é construído usando um st_file e um dicionário simples. Quando um st_dict é criado, há duas opções: ele pode ser criado vazio ou pode recuperar dados de uma execução abortada. Quando um st_dict é modificado, as modificações são refletidas tanto na versão na memória como também no st_file associado. Os observadores devolvem valores associados com o dicionário na memória.

\subsection{As Ações e os Processos}

Todos os aspectos da comunicação entre usuários e GPEs são tratados por primitivas de comunicação fornecidos por Concert/C. Cada uma das portas na especificação é de mão única, e cada uma foi implementada usando um par de funções: uma para enviar mensagem para a porta e uma outra para ler tais mensagens. As descrições dos dados das portas são descritas no arquivo ports.h (veja Apêndice I), e as funções usadas para acessá-las estão no arquivo gpeports.c (veja Apêndice J).

Os processos constituem a parte mais simples para implementar, já que os tipos de dados e a infraestrutura de comunicação já existia na hora de escrevê-los, como descrito nas seção anterior. Uma vez que os processos foram construídos usando funções que encapsulam detalhes das ações em Concert/C e objetos abstratos de alto nível (por exemplo, um dicionário estável, etc.) sua implementação foi uma tradução quase literal da sua especificação. As únicas maneiras pelas quais as funções se diferenciam são um resultado do requerimento de gerenciamento de memória e o fato de que a especificação está escrita em uma linguagem funcional, isto é, com recursão em vez de iteração e a falta de um comando para atribuição. As funções que implementam os processos estão nos arquivos gpemisc .c (veja Apêndice K) e gpemain.c (veja Apêndice L). 


\section{Execução dos Testes}

\subsection{O Teste dos $A D T \mathrm{~s}$}

Como mencionado no capítulo 9, a Membershiplist foi testada exaustivamente: Cada uma das operações foi aplicada com todas as possíveis combinações de parâmetros a todas as listas com tamanho menor ou igual a cinco. O driver computou os valores que correspondem aos dois lados dos casos teste e os comparou. A ferramenta ILDI de LITE foi utilizada como o oráculo para produzir os termos resultantes a serem verificados. Para simplificar os drivers dos testes, as operações foram testadas em grupos que correspondem aos seus valores de retorno. Todo o código do sistema (menos o código específico de Concert/C em gpeports.c) foi projetado para ser portável, e o desenvolvimento inicial foi feito em um PC e depois testado na rede do departamento usando o compilador de Concert/C.

O primeiro passo no teste do $A D T$ lista foi a geração das 326 listas. Os testes para add e remove usam uma função de igualdade que não faz parte da especificação. Essa função foi cuidadosamente implementada e testada usando uma hipótese de uniformidade e de regularidade de tamanho cinco, e portanto, a hipótese do oráculo (isto é, que está função está correta) parece razoável. Uma estratégia foi usada para testar os dicionários.

Surpreendentemente, nenhum erro foi encontrado nas implementações do dicionário e da lista. Isso não quer dizer que todos os testes deram resultados corretos nas primeiras execuções nas diferentes plataformas. Os drivers não foram especificados formalmente, e vários erros foram encontrados neles. A correção das listas e dos dicionários se deve provavelmente tanto a sua simplicidade como ao fato de que estes foram especificados formalmente. Quando o código executou corretamente no PC, esperou-se que o mesmo executasse corretamente nas estações de trabalho Sun. Isto não foi o caso. Depois de examinar o código para eliminar a possibilidade de erros óbvios, suspeitou-se de um bug no compilador. A execução de uma versão reduzida de um dos drivers confirmou esta hipótese: O programa deu saídas corretas com o compilador gnu mas não quando compilado com o compilador Concert/C. A fonte do erro foi identificada no preprocessador de Concert/C. A definição do tipo bool é

typedef enum $\{$ true $=(1==1)$, false $=(1==0)$ bool;

O pre-processador deveria produzir uma versão do programa com uma semântica equivalente àquela da entrada, mas em vez disso produziu

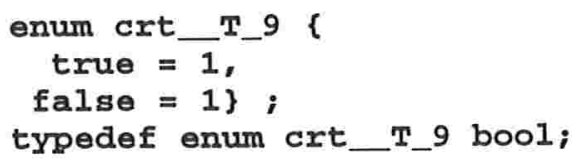

Esta tradução errada foi descoberta e foi evitada pela troca da definição de false para $(0==1)$. Esta experiência trouxe à luz um ponto muito importante: até as partes mais simples do código devem ser testadas, porque nunca se tem certeza do que o compilador faz com ela. Este problema não seria encontrado usando técnicas de verificação por demonstração porque seria assumido um compilador que funciona corretamente. O problema vem do fato de que $\mathrm{C}$ não tem um tipo Booleano, que teve que ser implementado através do typedef acima. A implementação é tão simples que a hipótese da sua correção foi feita. Se o compilador fosse um compilador correto de 
ANSI C, esta hipótese seria válida. Como isto não é o caso, e os drivers indicaram como erradas as implementações que estavam corretas.

Os quatro tipos adicionais discutidos na implementação que não fazem parte da especificação também não foram testados formalmente. Estes tipos foram testados de forma convencional e não são discutidos aqui.

\subsection{O Teste das Ações e dos Processos}

As ações atômicas da especificação consistem do envio de mensagens entre Users e GPEs e entre GPEs, e também modificam as estruturas de dados internas. Cada uma destas estruturas de dados foi implementada usando um $A D T$ previamente testado. A infra-estrutura de comunicação foi construída e testada passo a passo, começando com um sistema mínimo: inicialmente os processos GPE foram criados em vários hosts, e eles enviaram mensagens entre si para testar as conexões entre as GPE. Em seguida, pares de GPEs e usuários foram conectados para modelar mais fielmente o sistema final. Uma GPE enviou todas as mensagens recebidas para as demais GPEs, e enviou para seu usuário todas as mensagens recebidas das outras GPEs.

Uma vez que as facilidades de comunicação entre processos funcionaram corretamente, os processos baseados nelas puderam ser testados mais facilmente. Em vez de serem ligados com o arquivo objeto que contém as facilidades reais de comunicação, os drivers foram ligados com funções que imprimem o conteúdo das mensagens que seriam enviadas no sistema real.

Como ocorreu com os $A D T$ s, nenhum problema foi encontrado com a implementação dos processos transformacionais. Isso não foi uma surpresa, já que sua implementação é muito parecida com sua especificação. Cada função foi testada com seu próprio driver. Por exemplo, a função broadcast_to_GPEs foi testada com os casos teste gerados a partir do processo Broadcast (veja 9.2.1.). Para cada um dos seis experimentos de teste, um voteDictionary foi alocado e votos foram registrados, a função do broadcast foi chamada, e o dicionário foi liberado. O primeiro teste foi um caso degenerado: Uma mensagem de tipo Msgstatus é criada com um votedictionary vazio. A função foi chamada, e como previsto, nenhuma saída foi produzida. Embora esta função nunca seja chamada com tal entrada, ela mostrou-se funcionar corretamente mesmo neste caso.

Erros foram encontrados através dos testes no processo reativo GroupProtocol. Por exemplo, o primeiro caso teste (veja página 23) levou à descoberta de um erro no gerenciamento da memória e no tratamento dos time-outs, duas partes não especificadas da implementação. 


\section{Conclusões}

Neste trabalho especificou-se formalmente em LOTOS um sistema para a comunicação de grupo confiável. A validade de partes da especificação com relação a certas propriedades desejadas foi provada. A partir desta especificação foram gerados alguns casos teste para as principais estruturas de dados e funções do sistema. Tendo como base a especificação, foi também implementado o sistema para a rede de estações de trabalho Sun rodando UNIX e foram executados testes para várias partes do sistema.

O sistema implementado funciona, e ao que tudo indica está sem erro. O fato de que não há erros detectáveis usando os testes formais, combinado com a execução bem sucedida dos também importantes testes informais do sistema nos dá uma boa confiança na correção do sistema.

É sempre difícil coletar métricas do tempo gasto por um projeto em um ambiente acadêmico, mas pode ser interessante analisar o cronograma deste trabalho.

Dez. Começaram o estudo de LOTOS e a busca por ferramentas

Fev. Foram concluídas a especificação e sua explicação

Mar. Foram implementados e testados os ADTs

Abr. Encontrado um erro em Smile; validação dos recipientes com Loft

Maio Descoberta da ausência de Verif; iniciou-se a implementação dos processos

Jun. Terminou-se a derivação de testes para os processos

Jul. Terminou-se a implementação dos processos e executaram-se os casos teste Embora o processo tenha durado seis meses, a grande parte do tempo foi gasto lutando com as ferramentas. Assimilar as técnicas da geração de testes para processos reativos também foi trabalhoso.

Foi ilustrativo ver a diferença grande no tratamento formal das especificações de sistemas algébricos e as especificações de processos. Seria desejável que no futuro se obtivesse uma unificação das teorias e uma simplificação das mesmas. Embora as idéias chaves sejam similares, a terminologia usada por Gaudel em [Gau] e Brinksma e Tretsman em [Bri] e [Tre] é muito diferente e são usados métodos bem distintos para obter o mesmo objetivo. Consideramos este trabalho como um passo inicial.

Até mesmo não sendo possível usar todos as facetas dos métodos formais que existem, foi bom ver que tipo de benefício pode ser obtido com as poucas ferramentas existentes. Por exemplo, não foi possível encontrar nenhum erro na implementação dos $A D T$ s. Isto demonstra o valor de ter baseado a implementação em uma especificação formal. Mesmo que não seja possível fazer uma validação e verificação formal e completa para todo o sistema, acreditamos que o uso da especificação formal como base para a implementação e a seleção dos casos teste, bem como a utilização parcial de ferramentas para a validação, aumenta muito a confiabilidade e a qualidade do sistema final. Já a partir de uma simples especificação formal de um sistema obtém-se uma descrição mais abstrata, porém precisa, de um sistema, que pode ser usado para raciocinar matematicamente sobre o modelo do sistema dado pela especificação. Além disto, parecenos muito importante ter a possibilidade de testar se certas propriedades desejadas serão satisfeitas pelo sistema, examinando-se apenas a especificação formal antes da implementação do sistema. Mesmo que isto não seja possível, possíveis erros na especificação podem ser descobertos nas etapas seguintes, como aconteceu neste trabalho. Determinar se as saídas previstas para a especificação fazem sentido é muito importante neste caso. 
A nossa grande decepção com os métodos formais, em particular com os baseados em LOTOS, foi com a falta de ferramentas para trabalhar com as especificações. Não foi possível usar todas as ferramentas mencionadas na literatura, e daquelas utilizadas, foi decepcionante ver que elas não cumprem aquilo que os seus autores prometem. Foram descritas várias ferramentas na literatura que, ou ainda estão em desenvolvimento, ou existem mas não funcionam, ou existem mas não estão disponíveis para uso geral. Ao nosso ver, a ausência de ferramentas poderosas e de fácil uso é o maior obstáculo à adoção de uma metodologia de desenvolvimento de software baseada em métodos formais. Apesar de que o benefício que os métodos formais oferecem em termos de qualidade é enorme, o custo (em tempo) hoje em dia ainda é alto.

A fonte de uma grande parte do benefício do uso de métodos formais é a redundância inerente no processo de usá-los. Usando uma metodologia convencional de desenvolvimento de um sistema, o mesmo é descrito informalmente, a sua implementação baseada nesta descrição e testes são derivados de uma maneira ad hoc. Isto é muito menos rigoroso do que uma metodologia baseada em métodos formais. Já que a descrição é informal, há a possibilidade de se ter ambigüidade, lacunas na especificação ou uma má interpretação por parte do programador. Se a especificação fosse formal, seria possível modelar detalhadamente em uma linguagem de alto nível os aspectos importantes do sistema. Observamos que o tempo necessário para implementar um sistema a partir de uma especificação foi menor que seria a partir de uma descrição informal porque várias decisões de projeto são resolvidas no ato de especificar o sistema. Este fato é reportado por vários autores na literatura.

A partir do momento que existir um conjunto adequado de ferramentas para o trabalho de uma linguagem de especificação formal, o uso destas deverá se tornar comum no desenvolvimento de software, aumentando muito a qualidade e confiabilidade do mesmo. 


\section{Apêndice A: Informal Specification of a Reliable Group Communication Service}

\section{Introduction}

In the following we give an informal specification of a reliable group communication service (GS) for a static set of application processes. The aim of such a service is to provide an abstraction of broadcast communication for a group of application processes. The interface between the GS and the application processes consists of two communication channels fromUser and toUser, which are used to transmit the message data and status.

The primitives used to access these channels are

Bcast(\&SeqNr, message) is a non-blocking call by which the broadcast of message is requested. The sequence number $(S e q N r)$ and the identification of the requester uniquely identify every broadcast request.

Recv(\&MsgId, \&buffer, \&result) is a blocking call that returns only when channel toUser contains a message. When this occurs $8 M$ sgId, Ebuffer and Eresult are overwritten with the message identification, the message data and the result of the broadcast request. While the MsgId data structure contains the originator and the sequence number of the message, result contains the status of the request, which may be either committed or aborted. This primitive is used by the requesting application process to learn the outcomes of its previously broadcast messages.

The basic functionality of $G S$ is the following: For every broadcast request from an application process $G S$ will attempt to deliver the message to all group members (i.e. application processes). If any application process is not reachable or available, the broadcast is aborted and the message will not be delivered to any application process. In this case the broadcast is said to have been aborted. If all application processes are available, then the message is delivered to all application processes and the broadcast is said to have been committed.

The GS does not guarantee any order of message delivery, e.g. two causally dependent broadcast messages may arrive in different order at the individual application processes.

The GS implements message delivery with at-least-once semantics. This means that application processes may eventually receive duplicated messages, and since they have access to a system-unique MsgId, they are able to identify and discard them.

\section{Architecture}

The GS will be implemented by a static set of Group Protocol Entities (GPE), each serving a single application process, as shown in figure 2. For each broadcast request the GPE serving the application process that has issued the request will be elected the coordinator for the request. The coordinator is in charge of starting a Two-Phase-Commit Protocol among all GPE's in order to carry out an atomic 
broadcast. It will attempt to deliver the message to all GPE's, and if this is not possible, will not deliver the message to any of them.

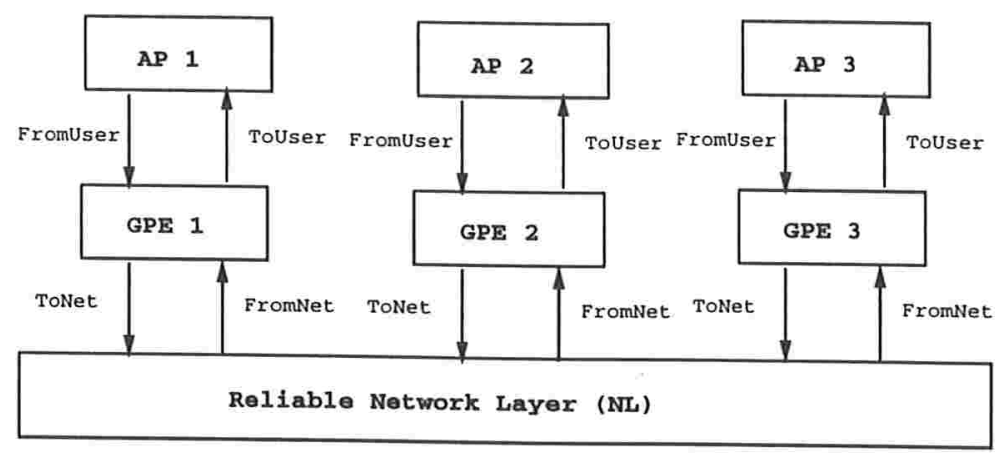

Figura 1: The GS Architecture

GPE's send messages to each other using the services of a Reliable Network Layer (NL), which guarantees message delivery but makes no assumption about message transmission times. The interface between every GPE and the underlying Network Layer consists of two communication channels fromNet and toNet, which are used to send peer-to-peer messages among GPE's.

Associated with each GPE is a non-volatile storage device, which is used to log the status of the individual broadcast requests. This allows GPE's which are recovering from a transient failure to resume the message delivery according to their state immediately before the failure.

\section{The Two-Phase Commit Protocol}

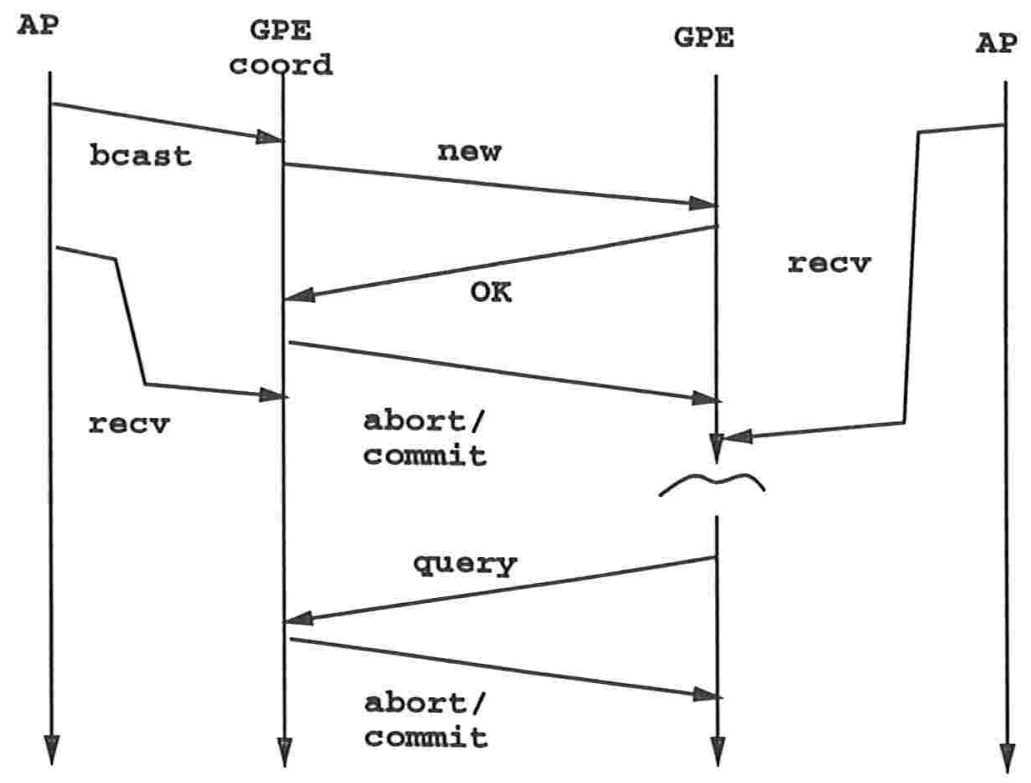

Figura 2: The Two Phase Commit Protocol

The Two-Phase Commit Protocol (2PCP), depicted in figure 2, can best be explained in two scenarios: 


\subsection{Normal processing}

Whenever a broadcast is received at channel fromUser of a GPE (which by this becomes the coordinator of the request), this request is logged as a new request and is sent to all other GPE's.

Upon arrival of a request with this status, all active GPE's log the request with status OK and respond to the coordinator with an OK message. For a certain period of time, the coordinator keeps collecting the replies. As soon as all of the replies are in, the message is changed to status commited. If time runs out and all replies have not been received, the message's status is changed to aborted. This status is logged, and the coordinator sends an outcome message (commited or aborted) to all other GPE's and then delivers the message to the process that made the request. Upon arrival of either a committed or aborted message, every GPE also updates this state of the request in its log. If the final state was committed, the message is delivered to the application process. Otherwise it is discarded, but remains in the log.

\subsection{Recovery}

When a GPE resumes after a failure, it first scans its log and does the following:

- For every message with state new it starts a new 2PCP in order complete the broadcast request of which it was the coordinator but had failed right after logging the request. All replies that have been received before the failure are lost.

- For every message logged with state OK it sends a query message to all GPE's to learn the final result of the request, since it apparently failed after replying to the request's coordinator with OK and before the arrival of the committed/aborted message. Since every GPE that has survived this second phase of the commit protocol has logged the result, all active GPE's will reply to the query with the outcome.

Meanwhile, the recovering GPE may assume its normal processing, i.e. it starts servicing its application process and receiving new broadcasts from the other GPE's while it is doing the recovery. This is only possible because GS does not give any delivery order guarantees.

\section{Failures}

At any moment one or more application processes and their corresponding GPE's may become unavailable for an arbitrary period of time. This also holds for the coordinator of a broadcast request.

Because we assume the Network Layer to be reliable, no failures can occur at message emission, message transmission and message delivery. This means that whenever a message is sent to a GPE via the toNet channel, this message will eventually be delivered by the NL to its correct destination. Moreover, no message corruption may occur in the network layer. Thus, our GS is only supposed to handle benign failures of the kind stop-fail.

We also disconsider any failures of the non-volatile media used to hold the current status of the broadcast requests, and in the access to this media by each GPE.

We further assume failure-atomicity in for following the set of actions at every GPE:

a) between accepting a broadcast request fromUser and logging of the request

b) between accepting a message from the network and logging it.

This failure-atomicity assumption is necessary to guarantee the consistency between the logs and the interactions of the GS with the application processes. 


\section{Additional Assumptions}

- Each GPE will have a fixed address GPEaddr which is known by all other GPE's at system configuration.

- The logs containing the status of the broadcast requests may be arbitrary long.

- There is a constant (and insignificant) delay for the search and access to any entry the logs (of any size).

- The channels toUser and fromUser are capable of holding an arbitrary number of messages, which are consumed in FIFO order.

\section{Desired Properties}

The following properties are expected from the GS described above:

Uniform consistency If a message is delivered to one application process it will eventually be delivered to all the remaining application processes.

Status Reachability Every broadcast request will eventually reach either the committ or abort state.

Status correctness(1) If a broadcast request becomes aborted, the corresponding message is not delivered to any application process.

Status correctness(2) If a broadcast request becomes committed it has been delivered to all the application processes.

Delivery guarantee If all nodes are available between the request of a broadcast and the delivery of its result to its requesting application process, then it has state committed.

No spontaneous broadcasts If a message is delivered to any application process, then there must have been a request for its broadcast.

Recoverability Every recovering GPE/application process will eventually receive all the committed messages that had been delivered to other application processes, provided that that at any time there is a sufficient number of active GPE's. (A sufficient but not necessary condition for this is one permanantly active GPE.) 


\section{Apêndice B: Especificação da GPE em LOTOS}

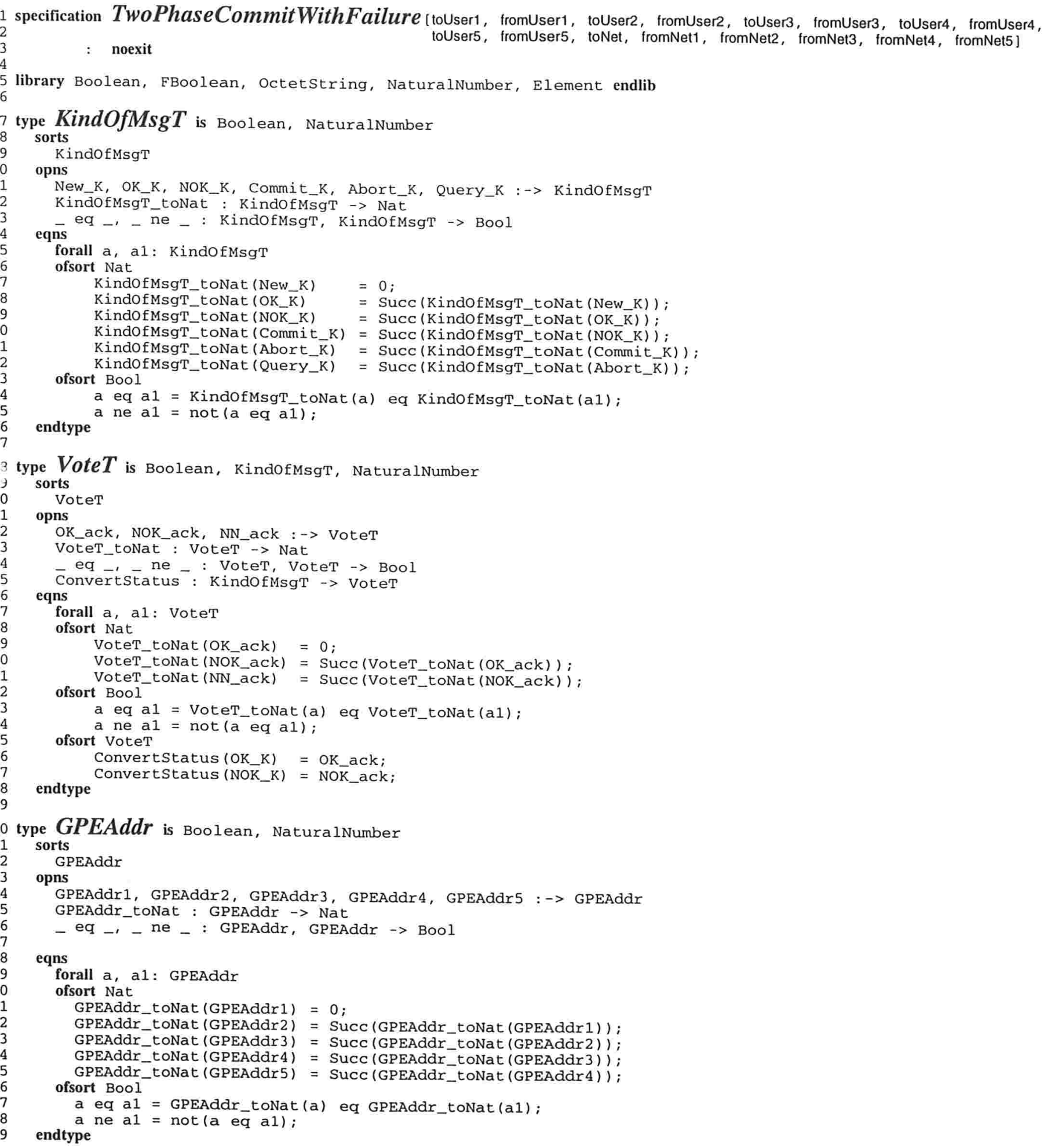




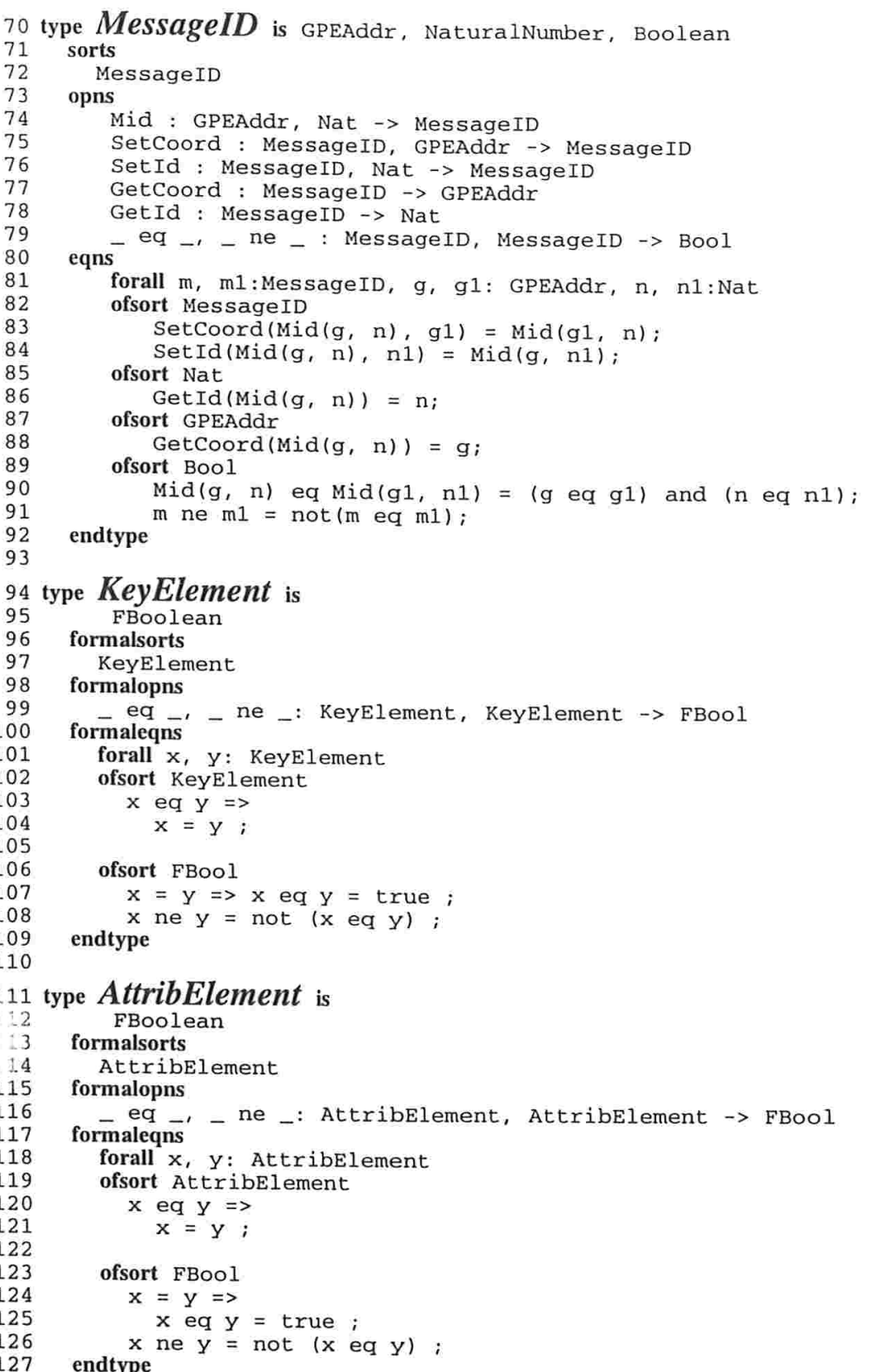

\title{
type VoteDictionary is
}

Container actualizedby VoteT, GPEAddr using

sortnames GPEAddr for KeyElement

VoteT for AttribElement

Bool for FBool

MembershipList for List

endtype

Votedictionary for Dictionary

\section{type MessageDictionary is}

\author{
Container actualizedby MessageID, MsgStatus using \\ sortnames MessageID for KeyElement \\ MsgStatus for AttribElement \\ Bool for FBool \\ MessageDictionary for Dictionary \\ MessageIDList for List
}


148 KeyElement, AttribElement, Boolean, NaturalNumber 
219 type MsgStatus is Boolean, NaturalNumber, Octetstring, GPEAddr, VoteT, KindofMsgT, VoteDictionary, MessageID
sorts

Msgstatus

opns

MsgS

SetData : Msgstatus, octetstring

Setseq

etcoora

setTo

SetFrom

SetKind

SetAcks

SetAck

SetAllAcks

GetData

Getseq

GetCoord

GetID

GetTo

GetFrom

GetKind

GetAcks

Msgstatus, Nat

$\rightarrow$ Msgstatus

$\rightarrow$ Msgstatus

Msgstatus, GPEAddr

Msgstatus, KindofMsg?

$\rightarrow$ MsgStatus

$\rightarrow$ Msgstatus

$\rightarrow$ Msgstatus

-> Msgstatus

Msgstatus, VoteDictionary $\rightarrow$ Msgstatus

Msgstatus, GPEAddr, VoteT $\rightarrow$ MsgStatus

MsgStatus, Membershiplist, VoteT $\rightarrow$ Msgstatus

: Msgstatus $\rightarrow$ Octetstring

Msgstatus $\rightarrow$ Nat

MsgStatus $\rightarrow$ GPEAddr

Msgstatus $\rightarrow$ MessageID

MsgStatus $\rightarrow$ GPEAddr

Msgstatus $\rightarrow$ GPEAddr

MsgStatus $\rightarrow$ KindofMsgT

: MsgStatus $\rightarrow$ VoteDictionary

equis

fo

: Msgstatus, MsgStatus $\rightarrow$ Bool

orall data: OctetString, seq: Nat, coord, to, from, gpe: GPEAddr, kind: KindofMsgT, ack : VoteT, acks: VoteDictionary, MS, MS1: MsgStatus, ML: MembershipList

ofsort Msgstatus

SetAck(MS, gpe, ack) = SetAcks (MS, Mod(GetAcks (MS), gpe, ack));

SetAllAcks(MS, ML, ack) = SetAcks (MS, Fill(GetAcks (MS), ML, ack))

ofsort Bool

MS eq MS1 = (GetCoord(MS) eq GetCoord(MS1)) and (GetSeq(MS) eq GetSeq(MS1)) MS ne MS1 = not (MS eq MS1)

ofsort Octetstring

GetData(SetData(MS, data)) = data;

GetData (SetSeq(MS, seq)) = GetData(MS)

GetData (SetCoord (MS, coord)) = GetData(MS)

GetData (SetTo(MS, to)) = GetData(MS);

GetData (SetFrom (MS, from)) = GetData(MS);

GetData (SetKind(MS, kind)) = GetData(MS)

GetData (SetAcks (MS, acks)) = GetData(MS)

ofsort MessageID

GetID(MS) = Mid(GetCoord(MS), GetSeq(MS));

ofsort Nat

GetSeq (SetData (MS, data)) = GetSeq(MS);

GetSeq (SetSeq(MS, seq)) = seq;

Getseq (SetCoord(MS, coord)) = GetSeq(MS);

GetSeq (SetTo (MS, to)) = GetSeq(MS);

GetSeq (SetFrom(MS, from)) = GetSeq(MS);

GetSeq (SetKind (MS, kind))

GetSeq (SetAcks (MS, acks)) = GetSeq (MS);

ofsort GPEAddr

GetCoord(SetData(MS, data)) = GetCoord(MS) ;

GetCoord (SetSeq(MS, seq)) = GetCoord(MS).

GetCoord (SetCoord (MS, coord) ) = coord;

GetCoord (SetTo(MS, to)) = GetCoord(MS);

GetCoord(SetFrom(MS, from)) = GetCoord(MS);

GetCoord (SetKind(MS, kind)) = GetCoord(MS):

GetCoord (SetAcks (MS, acks)) = GetCoord(MS);

GetTo (SetData (MS, data)) = GetTo(MS):

GetTo (SetSeq(MS, seq)) = GetTo(MS);

GetTo (SetCoord(MS, coord)) = GetTo(MS);

GetTo (SetTo(MS, to)) = to:

GetTo(SetFrom(MS, from)) = GetTo(MS) ;

GetTo (SetKind (MS, kind)) = GetTo(MS):

GetTo (SetAcks (MS, acks)) = GetTo(MS);

GetFrom (SetData (MS, data)) = GetFrom(MS);

GetFrom (SetSeq(MS, seq))

GetFrom (SetCoord(MS, coord)) $=\operatorname{GetFrom}(M S)$

GetFrom (SetTo(MS, to)) = GetFrom(MS).

GetFrom (SetFrom(MS, from)) = from;

GetFrom (SetKind(MS, kind)) = GetFrom(MS).

GetFrom (SetAcks (MS, acks)) = GetFrom(MS);

ofsort KindofMsgT

GetKind (SetData (MS, data)) = GetKind(MS):

GetKind (SetSeq(MS, seq)) = GetKind(MS):

GetKind (SetCoord(MS, coord)) = GetKind(MS)

GetKind (SetTo(MS, to)) = GetKind(MS).

GetKind (SetFrom(MS, from)) = GetKind(MS)

GetKind (SetKind(MS, kind)) = kind;

GetKind (SetAcks (MS, acks)) = GetKind(MS):

ofsort VoteDictionary

GetAcks (SetData (MS, data)) = GetAcks (MS):

GetAcks (SetSeq (MS, seq)) = GetAcks (MS)

GetAcks (SetCoord (MS, coord)) = GetAcks (MS):

GetAcks (SetTo(MS, to)) $=\operatorname{GetAcks}$ (MS)

GetAcks (SetFrom(MS, from)) = GetAcks (MS).

GetAcks (SetKind (MS, kind)) = GetAcks (MS): endtype

= acks; 
(* specification TwoPhase CommitWithFailureltoUser1, fromUser1, toUser2. fromUser2, toUser3, fromUser3, toUser4, fromUser4, *) to User5, from User5, toNet, from Net1, fromNet2, from Net 3, from Net4. fromNet5]

(* the membership list is static *) let AllMembers: MembershipList = Add (Add (Add (Add (Add (NIL, GPEAddr1), GPEAddr2), GPEAddr3), GPEAddr4), GPEAddr5
zero: Nat $=0$

in ( (User [toUser1, fromUser1] (zero) | [toUser1, fromUser1] |

(User [toUser2, fromUser2] (zero) | [toUser2, fromUser2] | (User [toUser3, fromUser3] (zero) | [toUser3, fromUser3] ] (User [toUser4, fromUser4] (zero) | [ toUser4, fromUser4] |

| [toNet, fromNet1, fromNet2, fromNet3, fromNet4, fromNet5] |

Network [toNet, fromNet1，fromNet2，fromNet3， fromNet4， fromNet5]

(*NB: this is the user as seen by the GPE; it is not a REAL user *)

process User[toUser, fromUser] (NumberofMsgssent:Nat) : noexit : =

$(*$ a user may start by either doing internal processing or receiving a message. after doing internal processing. the user may need to send a message $*$ ) [] $\mathrm{i}$;

( $? \mathrm{~m}$ : MsgStatus; User [toUser, fromUser] (NumberOfMsgsSent)

1] fromUser? User [toUser, fromUser] (NumberofMsgsSent) ) endproc $\left(*\right.$ User $\left.^{*}\right)$

$\left({ }^{*}\right.$ the network is point to point; it is NOT bus based *)

(* the network receives a message from its input gate and delivers it. Messages are never lost or corrupted *)

process Network [toNet, fromNet1, fromNet2, fromNet3, fromNet4, fromNet5]

: noexit :=

toNet ? msg : msgstatus;

( [getTo (msg) eq GPEaddr1] $\rightarrow$ fromNet1 ! msg; exit

[] [getTo(msg) eq GPEaddr2] $\rightarrow$ fromNet2 ! msg; exit

[] [getTo(msg) eq GPEaddr3] $\rightarrow$ fromNet3 ! msg; exit

[] [getTo (msg) eq GPEaddr4] $\rightarrow$ fromNet4! msg; exit

i] [getTo(msg) eq GPEaddr5] $\rightarrow$ fromNet5 ! msg; exit

I I Network [toNet, fromNet1, fromNet2, fromNet3, fromNet4, fromNet5] endproc (*Network*)

(* GPE has as parameters Myself and OtherMembers; keeps nonvolatile copies of Pending, Outcome, and Query Messages; and of course runs the group protoc. process $G P E$ [touser, fromUser, toNet, fromNet]

(Myself:GPEAddr, OtherMembers:MembershipList) : noexit :=

let emptyMessageDictionary : MessageDictionary = ED in

hide readPending, writePending, readoutcome, writeoutcome, readQuery, writeQuery in

( MessageFile [writePending, readPending ] (emptyMessageDictionary)

| MessageFile[writeoutcome, readoutcome ] (emptyMessageDictionary)

Messagefile[writeQuery, leadQuery l(emptyMessageDictionary)

)

| [writePending, readPending, writeoutcome, readoutcome, writeQuery, readQuery] |

GroupProtocolWithRecovery [toUser, fromUser, toNet, fromNet, readPending, writePending readoutcome, writeoutcome, readQuery, writeQuery]

where Myself, OtherMembers)

(* When a message dictionary is presented, it is stored; and when requested, the dictionary is presented *)

process Message File [inGate, outGate] (oldMD:MessageDictionary) : noexit :=

inGate? newMD:MessageDictionary; MessageFile [inGate, outGate] (newMD) [] outGate ! oldMD; $\quad$ MessageFile [inGate, outGate] (oldMD)
endproc (*PendingFile*) 
process GroupProtocolWithRecovery [toUser, fromUser, toNet, fromNet, readPending, writePending, readoutcome, writeoutcome, readQuery, writeQuery]

noexit : = (Myself:GPEAddr, OtherMembers:MembershipList)

GroupProtocol[toUser, fromUser, toNet, fromNet, readPending, writePending, readoutcome, writeoutcome, readQuery, writeQuery] (Myself, OtherMembers)

(* at any point, we can have a failure *)

[> Failure [toUser, fromUser, toNet, fromNet, readPending, writePending, readoutcome, writeoutcome, where readQuery, writeQuery] (Myself, OtherMembers) 
process GroupProtocol ${ }_{\text {[toUser, fromUser, toNet, fromNet, }}$ readPending, writePending, readoutcome, writeoutcome, readQuery, writeQuery]

: noexit : = (Myself:GPEAddr, OtherMembers:MembershipList)

(* read the pending, outcome, and query files *)

readPending ? Pending:MessageDictionary;

readoutcome? Outcome:MessageDictionary;

readQuery ? Queries:MessageDictionary;

( i ; exit

(* if there is a new message from the user to be sent to the group, put it in the pending list and broadcast it *)

[] fromUser? Msg:Msgstatus:

(let newMsg:MsgStatus=SetCoord(SetKind(Msg, New_K), Myself) in let

in writePending ! newPending; Broadcast [toNet] (newMsg, OtherMembers) $>>$ exit

[] fromNet ? Msg:Msgstatus ;

(* if the message is a ACK or a NAK then we need to store the vote and check to see if all the votes are in *)

( [ (GetKind(Msg) eq OK_K) or (GetKind(Msg) eq NOK_K)] ->

( let newPending:MessageDictionary = Mod(Pending, GetID(Msg)

SetAck (Retr(Pending, GetID(Msg)), GetFrom(Msg),

in ( writepending ! newPending; ConvertStatus (GetKind(Msg))))

IfCompleteSendResult [toNet, toUser, readPending, writePending, readoutcome, writeoutcome]

$$
>\text { exit }
$$
Myself, OtherMembers, Msg,

(* if this is a new message from another member, we need to send in our vote *)

[] [GetKind (Msg) = New $\mathrm{K}$ ] $\rightarrow$

(let $\mathrm{m}$ :MsgStatus $=$ SetKind $\left(\mathrm{Msg}, \mathrm{OK} \_\mathrm{K}\right)$ in let newPending: MessageDictionary=Mod (Pending, GetID(Msg), m)

in writepending ! newPending;

toNet ! SetFrom(SetTo(m, GetCoord(Msg)), Myself); exit

) )

[] [GetKind (Msg) =

(* that is, if it's in pending (it may happen because of the way we do queries) *)

( $[$ IsIn(Pending, GetID(Msg)) = true ] $\rightarrow$

( let newOutcome:MessageDictionary = Mod(Outcome, GetID(Msg), Msg), newPending:MessageDictionary = Remove (Pending, GetID (Msg)), newQueries:MessageDictionary = Remove (Queries, GetID(Msg))

in Writeoutcome! newOutcome; WriteQuery ! newQueries; toUser ! SetFrom(Msg, GetCoord(Msg)) : WritePending ! newPending;

) exit

(* if it's not, we can ignore it *)

[] [IsIn(Pending, GetID(Msg)) = false] $\rightarrow$ exit

(* if it's an abort we take it out of pending (if it's there) and discard it *)

[] $[$ GetKind (Msg) = Abort_K] $\rightarrow$

$([$ IsIn(Pending, GetID $(\mathrm{Msg}))=$ true $] \rightarrow$

( let newPending: MessageDictionary = Remove (Pending, GetID(Msg)), newOutcome: MessageDictionary = Mod(Outcome, GetID(Msg), Msg), newQuery :MessageDictionary = Remove (Queries, GetID(Msg))

in Writeoutcome! newoutcome; WriteQuery ! newQuery; WritePending ! newPending; exi

[] $[$ IsIn (Pending, GetID(Msg)) = false $] \rightarrow$ exit

[] [GetKind (Msg) = Query_K] $\rightarrow$

[IsIn (Outcome, GetID (Msg)) = true] $\rightarrow$

( let result:msgstatus = Retr (Outcome, GetID(Msg))

in toNet ! SetTo(result, GetFrom(Msg)); ) exit

[] [IsIn(Outcome, GetID(Msg)) = false] $\rightarrow$ exit

> readQuery ? Queries: MessageDictionary;

(1) ProcessQuesties [toNet] (Queries, OtherMembers) > []

> GroupProtocol[toUser, fromUser, toNet, fromNet, readPending, writePending, readoutcome, writeoutcome, where readQuery, writeQuery) (Myself, OtherMembers) 

process ProcessQueries [toNet] (Queries: MessageDictionary, otherMembers: MembershipList)
: exit :=

[] [IsEmpty (Queries) = false] $\rightarrow$ (let qk:MessageId=Choose(Queries) in let qm:Msgstatus=Retr(Queries, qk) in Broadcast (toNet] (SetKind (qm, QuerY_K), OtherMembers)

endproc (*ProcesQueries*)

process IfCompleteSendResult [ toNet, toUser, readPending, writepending, readoutcome, writeoutcome] : exit := (Myself:GPEAddr, OtherMembers:MembershipList, Msg:Msgstatus)

readPending ? Pending:MessageDictionary;

readoutcome? Outcome:MessageDictionary

(let $\mathrm{m}$ :Msgstatus = Retr (Pending, GetID(Msg))

in ([Count (GetAcks (m), OK_ack) + Count (GetAcks (m), NOK_ack) eq Length(OtherMembers) = false] $\rightarrow$ exit

[] [Count (GetAcks (m), OK_ack) + Count (GetAcks (m), NOK_ack) eq Length(OtherMembers) = true] $\rightarrow$ ( [Count (GetAcks (m), OK_ack) eq Length (OtherMembers) = true] $\rightarrow$

( let newPending:MessageDictionary = Remove (Pending, GetID(Msg)),

in writeoutcome ! newOutcome; toUser ! SetKind (Msg, Commit_K) : Broadcast [ toNet] (SetKind (Msg, Commit_K), otherMembers)

$>$ writePending ! newPending; exit

[] [Count (GetAcks (m), OK_ack) eq Length (OtherMembers) = false] $\rightarrow$ ( let newPending:MessageDictionary = Remove(Pending, GetID(Msg)) newOutcome: MessageDictionary = Mod(Outcome, GetID(Msg), SetKind(Msg, Abort_K))

in writeoutcome ! newOutcome; toUser ! SetKind(Msg, Abort_K) : Broadcast [ toNet] (SetKind (Msg, Abort_K), otherMembers)

$>$ writePending ! newPending; exit

$$
\text { ) }
$$

endproc (*IfCompleteSendResult *)

endproc (*GroupProtocol *)

(* While the GPE is down, it can either recover or stay down. If it is down, it takes available messages from the network.

Since LOTOS doesn't have a way to simulate timeouts, we have to use a work-around: If the message is a NEW, it is answered with a NOK. *) process Failure [toUser, fromUser, toNet, fromNet, readPending, writePending, readoutcome, writeoutcome, : noexit : := readQuery, writeQuery] (Myself:GPEAddr, otherMembers:MembershipList)

Recover [toUser, fromUser, toNet, fromNet, readPending, writePending, readoutcome, writeoutcome, readQuery, writeQuery ] (Myself, OtherMembers)

(]) ( i; Failure (toUser, fromUser, toNet, fromNet, readPending, writePending, readoutcome, writeoutcome readQuery, writeQuery] (Myself, OtherMembers)

[] fromNet? msg:Msgstatus;

( [GetKind(msg) eq New_K] $\rightarrow$ toNet ! SetKind(setTo(setFrom(msg, Myself), getCoord(msg)), NoK_K):

Failure [toUser, fromUser, toNet, fromNet, readPending, writePending, readoutcome, writeoutcome, readQuery, writeQuery] (Myself, OtherMembers)

[] [GetKind(msg) ne New_K] $\rightarrow$ Failure[toUser, fromUser, toNet, fromNet, readPending, writePending, readoutcome, writeoutcome, readQuery, writeQuery] ) (Myself, OtherMembers)

where

$(*$ Recovery reads the pending file, process it, and then continues with the main part of the protocol. *)

(* Of course, the recovery process can fail at any time $\left.{ }^{*}\right)$

process Recover [toUser, fromUser, toNet, fromNet, readPending, writePending, readoutcome, writeoutcome, : noexit : := readQuery, writeQuery] (Myself:GPEAddr, OtherMembers:MembershipList)

( readPending ? Pending:MessageDictionary;

ProcessPending [toNet, toUser, readPending, writePending, readoutcome

readQuery, writeQuery] (Pending, OtherMembers)
||| GroupProtocol [toUser, fromUser, toNet, fromNet, readPending, writePending, readoutcome, writeoutcome, readQuery, writeQuery] (Myself, otherMembers) (> Failure [toUser, fromUser, toNet, fromNet, readPending, writePending, readoutcome, writeoutcome,
readQuery, writeQuery] (Myself, OtherMembers) where 
process ProcessPending [toNet, touser, readPending, writePending, readoutcome, readQuery, writeQuery] : exit : : (Pending: MessageDictionary, OtherMembers:MembershipList)

$[$ IsEmpty (Pending) $=$ true $] \rightarrow$ exit

[] [IsEmpty (Pending) = false] $\rightarrow$

(* pk is the key of a message in the pending list, and pm is the message itself *)

( let pk:MessageID=Choose (Pending) in let pm:MsgStatus=Retr (Pending, pk)

$1^{*}$ if the message is an $O K$, we need to find out the outcome, so ask everybody, remember the queries, and process the rest of the messages

in ([GetKind (pm) eq OK_K] $\rightarrow$ readQuery ? qd:MessageDictionary:

writeQuery ! Mod (qd, pk, pm).

$\gg \quad$ Broadcast [toNet] (SetKind (pm, Query_K), OtherMembers)

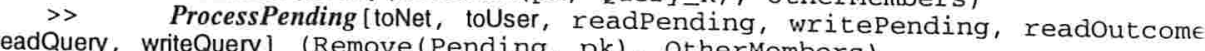

$\left(^{*}\right.$ if the message is a NEW, we need to send it again so we get a new set of votes. we also need to nullify any votes received *)

(* so far for this message, and finally process the rest of the messages in pending *)

[] [GetKind (pm) =New_K] $\rightarrow$

readoutcome ? Outcomes: MessageDictionary;

$($ [IsIn(Outcomes, pk) = true] $\rightarrow$ (Broadcast [toNet] (Retr (Outcomes, pk), otherMembers)

$\gg$ toUser ! Retr(Outcomes, pk);

ProcessPending [toNet, toUser, readPending, writepending, readoutcome, readQuery, writeQuery]

[ ] [IsIn(Outcomes, pk) = false] $\rightarrow$ (Broadcast [toNet] (pm, OtherMembers) > writePending ! Mod(Pending, pk,

SetAllAcks (pm, OtherMembers, NN_ack)) ;
ProcessPending (toNet, toUser, readPending, writePending, readoutcome, readQuery, writeQuery]

endproc (* ProcessPending *) endproc (*Recover *)

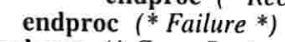

process Broadcast [toNet] (Msg:Msgstatus, toList:MembershipList)

$$
\text { [ exit : }=
$$

[] [IsEmpty (toList) $=$ false ${ }^{\rightarrow} \rightarrow$ ( let m:GPEAddr=Choose (tolist)

in toNet! SetTo (Msg, m);

endproc (* Broadcast $*$ )

Broadcast [toNet] (Msg, Remove (toList, m))

621 endspec (*TwoPhase CommitWithFailure *) 


\section{Apêndice C: A Rationale for the GPE Specification}

\section{Introduction}

The translation of the informal specification into LOTOS was not direct for several reasons. These fall into two categories: limitations of the LOTOS language and limitations of the tools that are available for working with LOTOS specifications.

The major limitation of LOTOS for us is that it doesn't have support for timeouts. The informal specification uses timeouts to detect failed GPE's. To overcome this shortcoming, we invented a message of status NOK that "failed" GPE's reply with when they receive broadcast messages. That is, we have failed GPE's continue to receive messages from the network and either ignore them or respond with NOK messages. When a process in our specification receives a NOK message, it should have the same effect as timing out in the implementation. Since we have this new message status in the specification that will not be present in the implementation, messages with this status must be created by some process in the specification, and we have the process Failure do this (see page A-12).

The inability to model timeouts also places some restrictions on the specification, such as requiring the atomicity of some sequences of actions for it to be valid. Even though these extra messages and atomicity restrictions will not be required of the final implementation, they are discussed below to ensure that they are taken into account during the validation of the specification.

Ideally, the formal specification would be as declarative as possible (describing what the system does) and not constructive (telling how it should do it). The tools that exist today cannot handle this approach in all cases, so we were obliged to overspecify some operations. An example of this is the definition of the choose operation in the list definition. choose should return an element of a nonempty list, so the behavior we want is

L eq NIL xor IsIn (L, choose $(L))=$ true.

We were forced instead to use something like

$\operatorname{choose}(\operatorname{con}(L, e))=e$,

which causes the selection of the last element added to the list. When implementing the system, we will use the declarative specifications described in this document instead of the LOTOS specifications. A discussion of the validity of the more declarative version is also included. Care should be taken when generating test cases to ensure that our test suite is unbiased (i.e., that correct implementations will not be rejected).

We have made a regularity assumption on the number of GPE's. Our specification models a set of five GPE's, their users, and the network that connects them. The users and network that are specified are only sophisticated enough to satisfy the minimal requirements of the GPE. For example, a user is only capable of receiving a message from its GPE and generating random messages to be broadcast to its group. Since this is all the GPE's need to know about what a user does, it is enough. 


\section{The ADT Definitions}

The two major sections of a LOTOS specification are the ADT specifications and the process specifications. The ADT section begins with three simple types that are suggested by the informal specification. Each of these is specified as a type that has operations to test for equality and inequality:

- KindofMsg

- VoteT several kinds of messages

- gPEAddr the three kinds of votes for the five GPE's in the system

The brute-force way of specifying the equality operation is $O\left(n^{2}\right)$ in the number of equations, where $n$ is the number values in the sort. An alternate way was suggested in the Lite User's Manual ([Can], 40-43) that is $O(n)$. An auxiliary function is introduced that converts the values of the type to sort nat, and the equality operation compares these converted numerical values. The vote type also has an operation to convert the kind of messages oK and NOK to the Vote type.

Users will be able to uniquely identify messages by means of the sender and a sequence number. This is expressed in the MessageID type, which has operations to set and retrieve these two values.

\subsection{Containers}

The two kinds of containers that are used in the specification are Lists and Dictionaries (also known as associative arrays) (see page A-7). The containers that we specify are generic, in that they describe the storage and retrieval of elements of formal sorts and are later instantiated with actual sorts. They can be instantiated with any sorts that offer the eq and ne operations. The formal types used are KeyElement and AttribElement (see page A-6). KeyElement is the type of the values stored in a list and that are used as the indices into a dictionary. AttribElement is the type of the values associated with the keys in the dictionary.

Again we were forced away from a preferred specification because of the tools available. One operation, Fill, operates on both a list and a dictionary. We would like to have had the two kinds of containers defined as different types, but we were forced to combine them as two sorts within the same type because of the type resolution capabilities of our tools. Even so, it was possible to textually separate them. They cannot be instantiated separately. Whenever a dictionary is instantiated, a list of the key type is also produced. This is not necessarily bad, because in one of the two types of dictionaries that we instantiate (the VoteDictionary), we want to have type that happens to be a list of the key type. The collections that are suggested by the informal specification and instantiated in the specification are

- VoteDictionary to store the votes cast by each GPE for a given message

- MessageDictionary a message log

- Membershiplist to store the network addresses of the GPE's in the system (defined in VoteDictionary)

\subsubsection{Lists}

The simpler of the two containers is the List, and the simplest list is NIL-an empty list. The operations Add and Remove are used to add and remove elements from a list. The boolean operations IsIn, IsNotIn, and IsEmpty return information about a list, and Length returns the number of elements in the list. Choose returns an element of the list. 
We wanted lists in which duplicates are not allowed, so Add operation only has an effect when the element to be added isn't already in the list. To specify this using Add as a generator would have resulted in axiomatizing a generator, which isn't allowed by the tools. We introduced an auxiliary operator con that is the true generator, but which is not a part of the public interface to the sort and should not be used elsewhere.

Remove eliminates all traces of a given element from the list. The result of removing an element from NIL is just NIL. The result of removing an element from a list made up of at least one addition has two possibilities: either the element added is the element to be removed or it is not. If it is, the result is the list that results from removing the element from the rest of the list. If it's not, it's the list formed by adding the list element back to the list that's formed by the removal of the element from the rest of the list. This is another example of the type of overspecification we were forced into. The property we are interested in is IsIn (Remove (L, e), e) = false.

No element Is In the empty list. If the list is not empty, each element that was added must be check to see if it's equal to the one sought. If it is, then the value is true. If it's not, the rest of the list must be searched. IsNot In is the negation of IsIn.

A list is Empty if its Length is zero, where Length returns the number of elements in the list. The length of NIL is zero, and each Con increases the length by one.

The problem with the definition of the choose operation was discussed in the introduction. Choose (NIL) is not defined.

\subsubsection{Dictionary}

A Dictionary is an associative array; that is, it is an array generalized to take any type as indices. Its operations are summarized below.
- $E D$
the empty dictionary
- Mod, Fill
add key/attribute pairs
- Remove
eliminates a given key
- Retr
- Choose
- IsIn, IsNotIn, IsEmpty retrieves the attribute associated with a given key gives a key
- Size give membership information
- Count returns the number of key/attribute pairs returns the number of occurrences of a given attribute

There is also a con operation that is not part of the interface that was added for the same reasons as it was added to the List sort.

The Mod and Remove operations are similar to the list's Add and Remove except that they work with key/attribute pairs. If the key to be Modified is already in the dictionary, it and its associated attribute are removed before the new pair is added. Fill is similar to Mod, except that it takes a list of keys to be set to the same attribute value. It is used only once in the specification, in the type Msgstatus when we want to set the votes from all other GPE's for a given message to NN_ack.

\subsection{MessageStatus}


The messages the GPE's work with include more than just the information given by their users. They also contain control information, and all of this is stored in the data type Messagestatus. Its various fields are summarized below.

- Data the data a user wants to be broadcast

- Seq the message's sequence number

- Coord the GPE acting as coordinator for this message

- To the GPE this message is being sent to

- From the GPE this message is being sent from

- Kind the kind of this message

- Acks the dictionary of votes for this message

Each of these fields has Set- and Get-operations. The constructors for this type are MsgS and the Setoperations. While this way of specifying a record structure uses more space than others, it gives a better model of undefined fields.

Only the coordinator of a message should access the Acks. To facilitate working with this field, two additional operations are provided: SetAck, which sets a the AckT for a single GPEAddr, and SetAllAcks, which sets the AckTs for an entire MembershipList.

\section{The Process Definitions}

The gates that the GPE uses to communicate with its environment are listed on lines 1 and 2 of the specification. Other gates are used in the specification, but they are not visible from the outside.

The MembershipList AllMembers is a list of all of the GPEAddresses in the system.

The behavior of the system is the interplay of the users, GPE's, and the network. Each of the five Users has a GPE that it uses to coordinate its group activities. All of the users and GPE's run in para parallel and communicate through the toUser $r_{i}$ and fromUser ${ }_{i}$ gates. The GPE's communicate with each other by sending messages through the Network. As mentioned in the introduction, the network and users of the specification are not intended to model real users and network. Their only purpose is to give the GPE a context in which to operate, showing the interface that the GPE assumes to be in place for to communicate with the outside world.

If a message if available from its GPE, a User can choose to take it. If there is none (or if the user chooses not to take it) it can either generate a message to be broadcast to the group or not. Users also generate the message sequence numbers. In the implementation this will be taken care of by the BCast function, as described in the informal specification (see page A-1).

The Network is always ready to receive messages from the GPE's. Once one is sent, the Network sends it to its destination. The Network is reliable, so there is no chance of loosing or corrupting messages. The recursive call (line 362, page A-9) is made in parallel with the delivery to prevent the system from deadlocking.

A GPE is responsible for implementing the two-phase commit protocol. To do this it needs to keep various logs, which are implemented as message Files. These files are local and private to each GPE. Their functionality is simply storing a dictionary of Messagestatus, updating it when a new dictionary is 
presented and returning the last version stored when asked for it. In this simplified version, the entire log is read and written each time the file is accessed. The three files are the Pending file, the Outcome file, and the Query file, and their purposes are summarized bellow:

- Pending Stores messages whose outcomes are unknown.

- Outcome Stores the results of messages, either aborted or committed.

- Query Stores queries that a GPE has made but not received replies to.

The Group Protocol with Recovery process shows that while executing the protocol, failure can occur at any time. The Recovery process is covered after the discussion of the Failure process. No information, except that stored in the files, is available after a Failure.

The Group Protocol starts by reading the log files into memory. When the system was booted for the first time, the Pending, Outcome, and Query files were created as empty. The Query file is created empty each time the system recovers from failure. The Group Protocol specification has three main parts. The first (and by far the largest) is handling a message. The second is processing the entries in the Query log. The final step is just a recursive call to process further messages.

In the first part, the protocol has three choices: do internal processing (that is invisible to the environment), receive a message from the user, or receive a message from the network. If the choice is to receive from the user, the message must be a request for a group broadcast. The protocol requires that this new message be recorded in the Pending file before the broadcasts are started. Failure is not allowed between the time of the receive and writing in the log for the specification to be valid. A way must still be found to overcome this in the implementation. The timer in the implementation should be started after the message has been sent to the other GPE's.

If the choice in the first part is to receive a message from the network, there are several possibilities, depending on the Kind of the message. If it is an oK or NOK, then this message is a vote for a previously broadcast message. This vote is then stored in the pending log. After storing the vote, the system checks to see if all the votes are in, and if so, sends the result. This last process is described in more detail below. In the implementation, the NOK messages will not exist. Instead of only checking the vote count when a message arrives, there will also be a timer that is started when the message is broadcast. If all of the votes (which will be OK's) are not received within a cutoff limit, the delivery will be considered a unsuccessful and the other GPE's will be sent an Abort message. In the formal specification, NOK thus simulates a response that arrived after the before-mentioned timeout.

If the message received is a New then it is stored in the pending file and an oK vote is sent. The log entry must be recorded before the reply is made to ensure that the GPE can determine the result if it fails before receiving the result. (Consider this scenario: This GPE fails after the acknowledging and before logging, and the result of the message was Commit after recovering. The GPE wouldn't know that the message existed, and so could neither deliver it to the user of query its outcome.)

For the specification to be valid, failure is also not allowed between receiving the message and storing it in the Pending log. This is an result of the NOK messages that we have introduced, and will not be required in the implementation. Consider if this were not the case: Because of a failure at this point, the message would have been lost without a trace by the failing GPE, and the coordinator will never receive a (possibly negative) acknowledgement. Therefore, this message would never be committed or aborted. In the implementation, a failure at the above mentioned point will eventually cause a timeout, the message will be aborted, and no harm is done. 
Upon receiving either a Commit or an Abort message, the GPE first determines if it's in the Pending log. If not, the receipt of the outcome is a duplicate and can be ignored. (Duplicates are possible because of the way the Query log is processed). If the message is still in the Pending log, the GPE records the Outcome in a log and, if it's in the Query log, removes it. Commit messages are passed to the user while Aborts are not. The message is finally removed from the Pending log. The order must be the above for proper recovery from a failure.

The final kind of message is the Query. When received, the outcome, if known, is sent.

Since timeouts aren't available, Processing the Query log is done by a nondeterministic choice in the specification. In the implementation, it should of course be triggered by a timeout. The Query file is created during the Recovery process, and should be processed periodically until empty. It consists simply of resending the Query messages that were first sent during the last Recovery process. In the implementation, a timer should be started after sending the last Query, but only if the log is not empty.

IfCompleteSendResult starts by comparing the number of votes received so far with the number of GPE's that the message was sent to. If not, the process exits. If so, the result must be determined. If all of the votes are OK's, then the message is committed. If not, it is aborted. In the implementation, there are no NOK votes, so only the first check need be made. If all of the votes are in, then the message can be committed. An Abort will only be sent when the message times out.

In reality, when a node fails it does nothing. If we could control such a thing so as to write code for its implementation, we would just eliminate the possibility of its occurring. Since we have invented the NOK messages, we must generate them, and they are only generated when the GPE has failed. We are then obliged to include a Failure process. At any moment a failed process can Recover. If it doesn't recover, it can take an arriving message from the network. If the message is New, a NOK must be sent. Any other kind of message is thrown away.

When a GPE Recovers from failure, it must process its Pending log. This is done in parallel with continuing its normal operation. A recovering GPE is not immune to failure, and can indeed fail at any time (with the above atomicity limitations).

To Process the Pending log, we iterate through it. Only messages of types New and oK are stored in the in the Pending log. For New messages, we discard any votes that were received before the failure and start the group broadcast process from scratch. OK messages require less work. All we do is ask the other GPE's for the outcome, if we don't already have it stored in the Outcome log.

To Broadcast a message, all we do is iteratively send it to each of the other GPE's in the system. 


\section{Apêndice D - O Conjunto Original para a Validação dos Containers}

O processo de validação é ad hoc, mas uma maneira sistemática foi usada na criação das propriedades pela consideração dos pseudo-construtores com construtores. Para listas, isto significa que serão demonstrado que NIL, Add e Remove tem o comportamento correto em combinação com si e com os demais operações. Validação consiste não somente da demonstração de que uma operação faz o que deve, mas também que não faz nada que não deve.

\section{Tipo List}

\section{Operação Add}

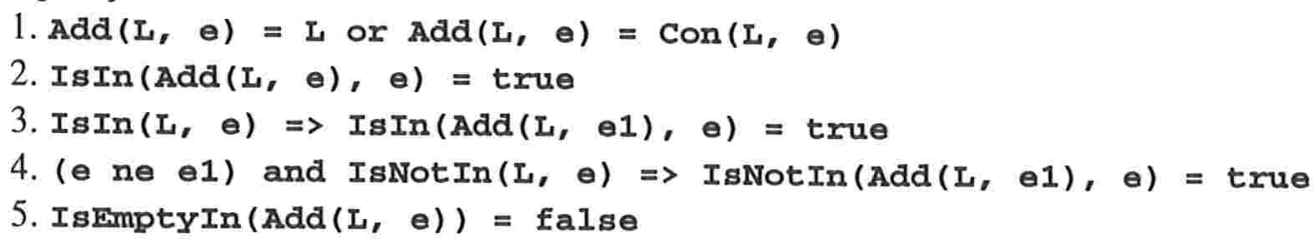

\section{Operação Remove}

1. $\operatorname{IsIn}($ Remove $(L, e), e)=$ false

2. (e ne e1) and $\operatorname{IsIn}(L, e) \Rightarrow \operatorname{IsIn}(\operatorname{Remove}(L, e 1), e)=$ true

3. $\operatorname{IsNotIn}(L, e) \Rightarrow \operatorname{IsNotIn}(\operatorname{Remove}(I, e 1), e)=$ true

Operações IsIn e IsNotIn

1. $\operatorname{IsIn}(I, e) \Rightarrow \operatorname{IsNotIn}(L, e)=f a I s e$

2. $\operatorname{IsNotIn}(I, \theta) \Rightarrow \operatorname{IsIn}(L, \theta)= \pm a I s e$

Operação Choose

1. (L eq NIL) xor IsIn(L, Choose(L)) = true

Operação Length

1. (Length (Add( $L, \theta)$ ) eq Length(L)) or (Length(Add(L, e)) eq Succ(Length(L)))

2. $\operatorname{IsIn}(I, e) \Rightarrow \operatorname{Length}(\operatorname{Add}(L, e))$ eq Length(L)

3. $\operatorname{IsNotIn}(L, e) \Rightarrow \operatorname{Length}(\operatorname{Add}(L, \Theta))$ eg $\operatorname{Succ}(\operatorname{Length}(L))=\operatorname{true}$

4. Length (Remove (L), e) le Length (L)

5. $\operatorname{IsIn}(L, e) \Rightarrow \operatorname{Length}(\operatorname{Remove}(L, e)$ ) It Length (L)

6. IsNotIn ( $L, e) \Rightarrow \operatorname{Length}(\operatorname{Remove}(L, e)$ ) eq Length(L)

Operações IsEmpty e IsNotEmpty

1. (I eq NIL) sor IsNotimpty(L) = true

2. IsEmpty (I) $\Rightarrow$ IsNot Empty (L) = false

3. IsNotEmpty $(L) \Rightarrow$ IsEmpty $(L)=$ false 


\section{Tipo Dictionary}

Operação Mod

1. $\operatorname{IsIn}(\operatorname{Mod}(D, k, e), k)=$ true

2. $\operatorname{Retr}(\operatorname{Mod}(D, k, e), k)=e$

3. $\operatorname{IsIn}(D, e 1) \Rightarrow \operatorname{IsIn}(\operatorname{Mod}(D, k, e), e 1)=$ true

4. ( $k \mathrm{ne} k 1)$ and $\operatorname{IsNotIn}(D, k 1) \Rightarrow \operatorname{IsNotIn}(\operatorname{Mod}(D, k, e), k 1)=$ true

5. IsEmpty $(\operatorname{Mod}(D, k, e))=$ false

Operação Remove

1. IsIn (Remove $(D, k), k)=$ false

2. (k ne k1) and $\operatorname{IsIn}(D, k 1) \Rightarrow \operatorname{IsIn}(\operatorname{Remove}(D, k), k 1)=$ true

3. $\operatorname{IsNotIn}(D, k 1) \Rightarrow \operatorname{IsNotIn}(\operatorname{Remove}(D, k), k 1)=$ true

Operação Fill

1. IsIn(Fill(D, L, e), Choose(L)) = true

2. $\operatorname{Retr}(F i l l(D, L, e)$, Choose(L)) =e

3. $\operatorname{IsIn}(D, e 1) \Rightarrow \operatorname{IsIn}(F i l l(D, L, e))=\operatorname{true}$

4. IsNotIn(L, k) and $\operatorname{IsNotIn}(D, k) \Rightarrow \operatorname{IsNotIn}(F i I l(D, L, e), k)=$ true

5. IsNotEmpty $(L) \Rightarrow$ IsNotEmpty $(F i l l(D, L, e))=$ true

Operação Choose

1. $\operatorname{IsIn}(D$, Choose (D)) = true

Operações IsIn e IsNotIn

1. $\operatorname{IsIn}(D, k) \Rightarrow \operatorname{IsNotIn}(D, k)=f a l s e$

2. $\operatorname{IsNotIn}(D, k) \Rightarrow \operatorname{IsIn}(D, k)=f a I s e$

Operaçõe IsEmpty e IsNotEmpty

1. (D = ED) xor IsNotempty (D)

2. IsEmpty (D) $\Rightarrow$ IsNotEmpty $(D)=$ false

3. IsNotEmpty (D) $\Rightarrow$ Ismpty $(D)=$ false

Operação Size

1.(Size(Mod(D, k, e)) eq $\operatorname{Size(D))~or~}(\operatorname{Size}(\operatorname{Mod}(D, k, e))$ eq $\operatorname{Succ}(\operatorname{Size}(L)))$

2. $\operatorname{IsIn}(D, k) \Rightarrow \operatorname{Size}(\operatorname{Mod}(D, k, e))$ eq $\operatorname{Size}(D)$

3. $\operatorname{IsNotIn}(D, k) \Rightarrow \operatorname{Size}(\operatorname{Mod}(D, k, e))$ eq $\operatorname{Succ}(\operatorname{Size}(L))=$ true

4. Size(Fill(D, L, e)) ge Size(D)

5. Size(Fill(D, L, e)) le (Size(D) + Length(L))

6. Size(Remove(D), k) le Size(D)

7. $\operatorname{IsIn}(D, k) \Rightarrow \operatorname{Size}(\operatorname{Remove}(D, k))$ It $\operatorname{size}(D)$

8. IsNotIn(D, k) $\Rightarrow \operatorname{Size}(\operatorname{Remove}(D, k)$ ) eq Size(D)

Operação Size

1. Count (D, e) le Size $(D, e)$ 


\section{Apêndice E: Especificação dos Containers em PLUSS}

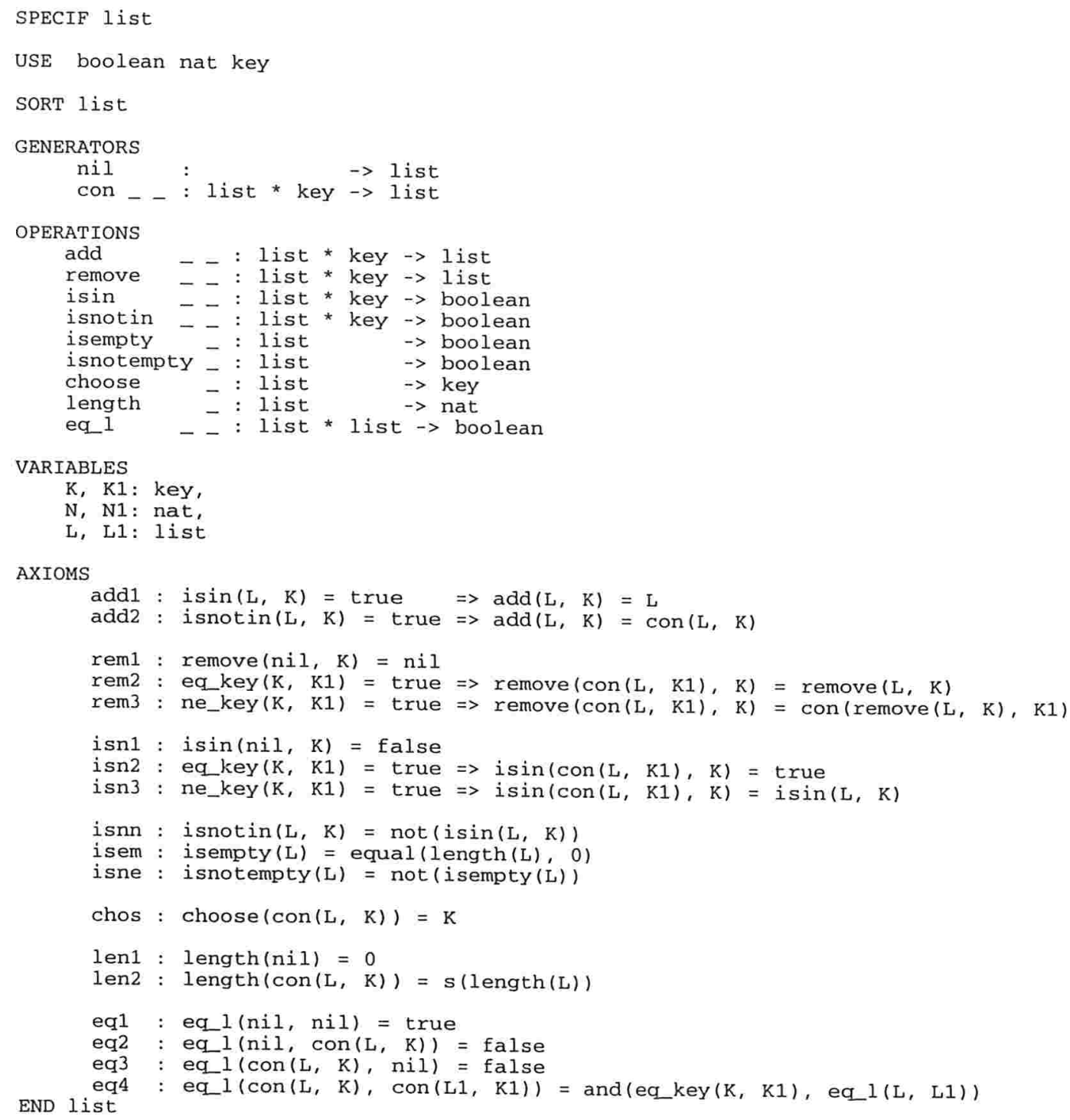


SPECIF dictionary

USE boolean nat list key attrib

SORT dictionary

GENERATORS

ed
con _ _ $\quad$ : dictionary * key * attrib $\rightarrow$ dictionary

OPERATIONS

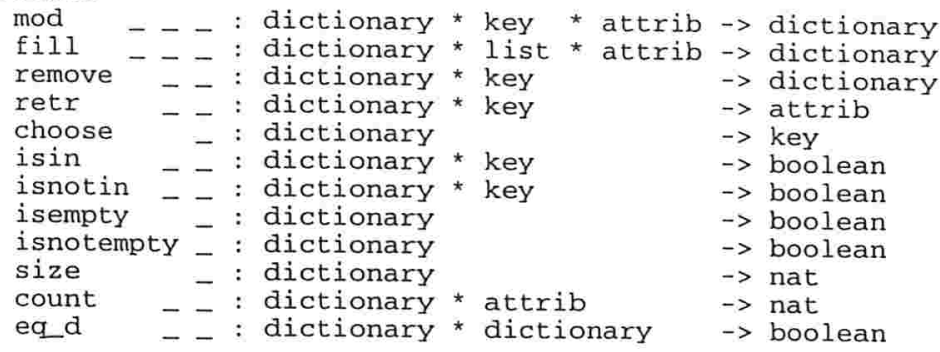

VARIABLES

K, K1 : key,

E, E1 : attrib,

$\mathrm{N}, \mathrm{N1}$ : nat,

L : list,

D, D1 : dictionary

AXIOMS

$\bmod 1: \operatorname{isin}(D, K) \quad=$ true $\Rightarrow \bmod (D, K, E)=\operatorname{con}($ remove $(D, K), K, E)$

$\bmod 2: \operatorname{isnotin}(D, K)=\operatorname{true}=\bmod (D, K, E)=\operatorname{con}(D, K, E)$

rem1 : remove $(e d, K)=$ ed

rem2 : eq_key $(K, K 1)=\operatorname{true} \Rightarrow \operatorname{remove}(\operatorname{con}(D, K 1, E), K)=\operatorname{remove}(D, K)$

rem3 : ne_key $(K, K 1)=\operatorname{true}=>\operatorname{remove}(\operatorname{con}(D, K 1, E), K)=\operatorname{con}(\operatorname{remove}(D, K), K 1, E)$

fill : fill (D, nil, E) $=D$

fil2 : $f i l l(D, \operatorname{con}(L, K), E)=f i l l(\bmod (D, K, E), L, E)$

ret1 : eq_key $(K, K 1)=\operatorname{true} \Rightarrow \operatorname{retr}(\operatorname{con}(D, K 1, E), K)=E$

ret2: ne_key $(K, K 1)=\operatorname{true} \Rightarrow \operatorname{retr}(\operatorname{con}(D, K 1, E), K)=\operatorname{retr}(D, K)$

cho1 : $\operatorname{choose}(\operatorname{con}(D, K, E))=K$

isn $1:$ isin(ed, $K)=$ false

isn2 : eq_key $(K, K 1)=\operatorname{true}=>\operatorname{isin}(\operatorname{con}(D, K 1, E), K)=$ true

isn3 : ne_key $(K, K 1)=\operatorname{true}=>i \sin (\operatorname{con}(D, K 1, E), K)=i \sin (D, K)$

isn4: isnotin(D, K) $=\operatorname{not}(i \sin (D, K))$

ism1 : isempty $(D)=$ equal $(\operatorname{size}(D), 0)$

ism2 : isnotempty (D) $=$ not (isempty (D)

siz1 : size (ed) $=0$

$\operatorname{siz2}: \operatorname{size}(\operatorname{con}(D, K, E))=s(\operatorname{size}(D))$

cnt1: $\operatorname{count}(e d, E)=0$

$\operatorname{cnt2}:$ eq_attrib $(E, E 1)=\operatorname{true}=>\operatorname{count}(\operatorname{con}(D, K, E 1), E)=s(\operatorname{count}(D, E))$

$\operatorname{cnt3}:$ ne_attrib(E, E1) $=\operatorname{true}=\operatorname{count}(\operatorname{con}(D, K, E 1), E)=\operatorname{count}(D, E)$

eqd1 : eq_d (ed, ed) = true

eqd2 : eqda(con $(D, K, E)$, ed) = false

eqd3 : eq_d (ed, $\operatorname{con}(D, K, E))=$ false

eqd4: eq_d $(\operatorname{con}(D, K, E), D 1)=\operatorname{and}(\operatorname{and}(i \sin (D 1, K)$, eq_attrib(retr $(D 1, K), E))$, eq_d(D, remove $(D 1, K)))$ END dictionary 


\section{Apêndice F: Conjunto de Propriedades para a Validação dos Containers}

As seguintes propriedades foram fornecidas à ferramenta LOFT.

1. Conjunto de Propriedades para Iist.spec

As propriedades sem anotação foram demonstradas para $\alpha=10$. Aquelas marcadas com $\dagger$ foram demonstradas para $\alpha=5$. Nenhum resultado significativo foi obtido para aquelas anotadas com $\ddagger$.

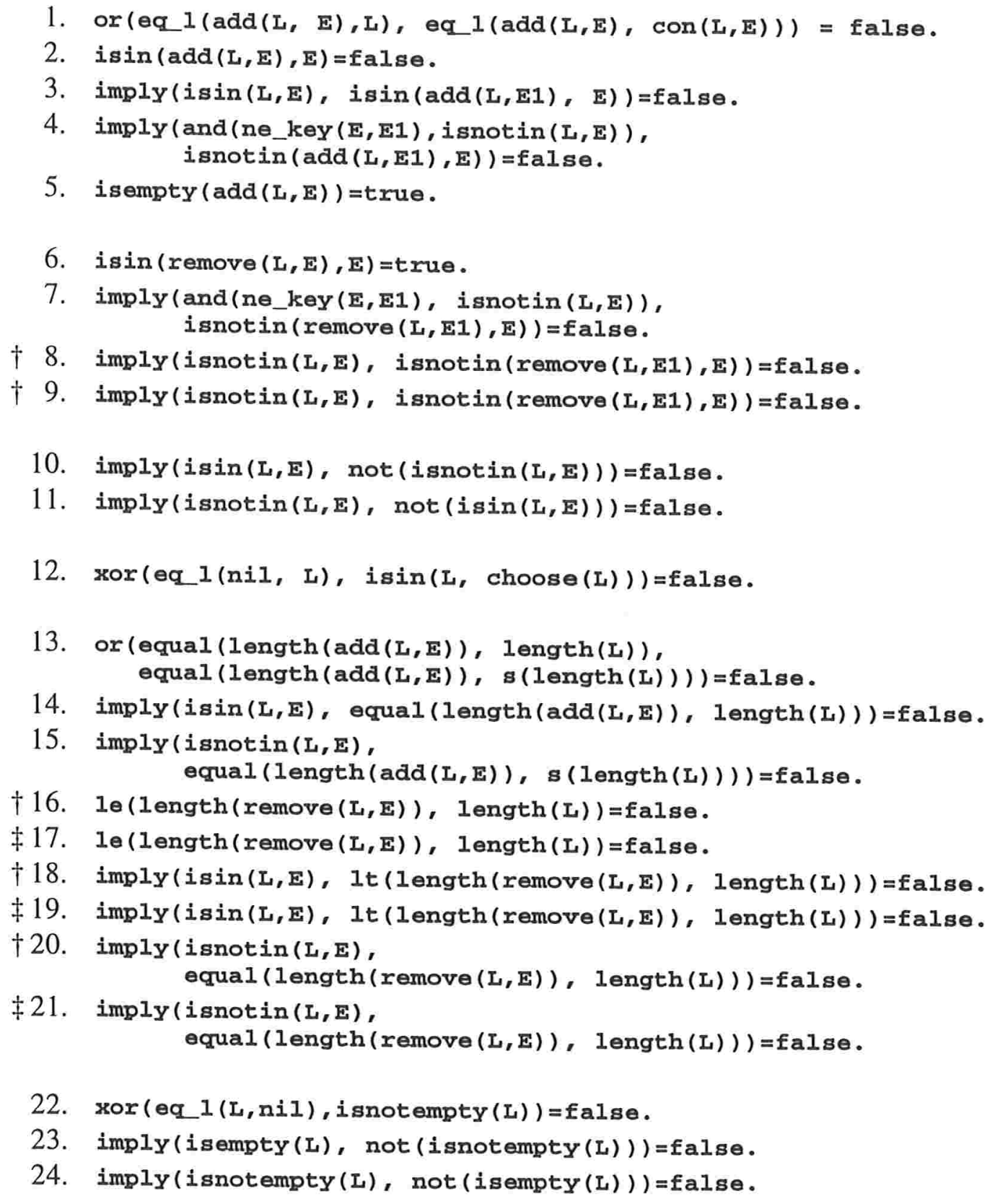

22. $\operatorname{xor}($ eq $1(L, n i l)$, isnotempty $(L))=$ false.

23. imply (isempty (L), not (isnotempty $(L)))=$ false.

24. imply (isnotempty $(L), \operatorname{not}(i \operatorname{sempty}(L)))=$ false. 
1. Conjunto de Propriedades para dict.spec

As propriedades sem anotação foram demonstradas para $\alpha=5$. Nenhum resultado significativo foi obtido para aquelas anotadas com $\ddagger$.

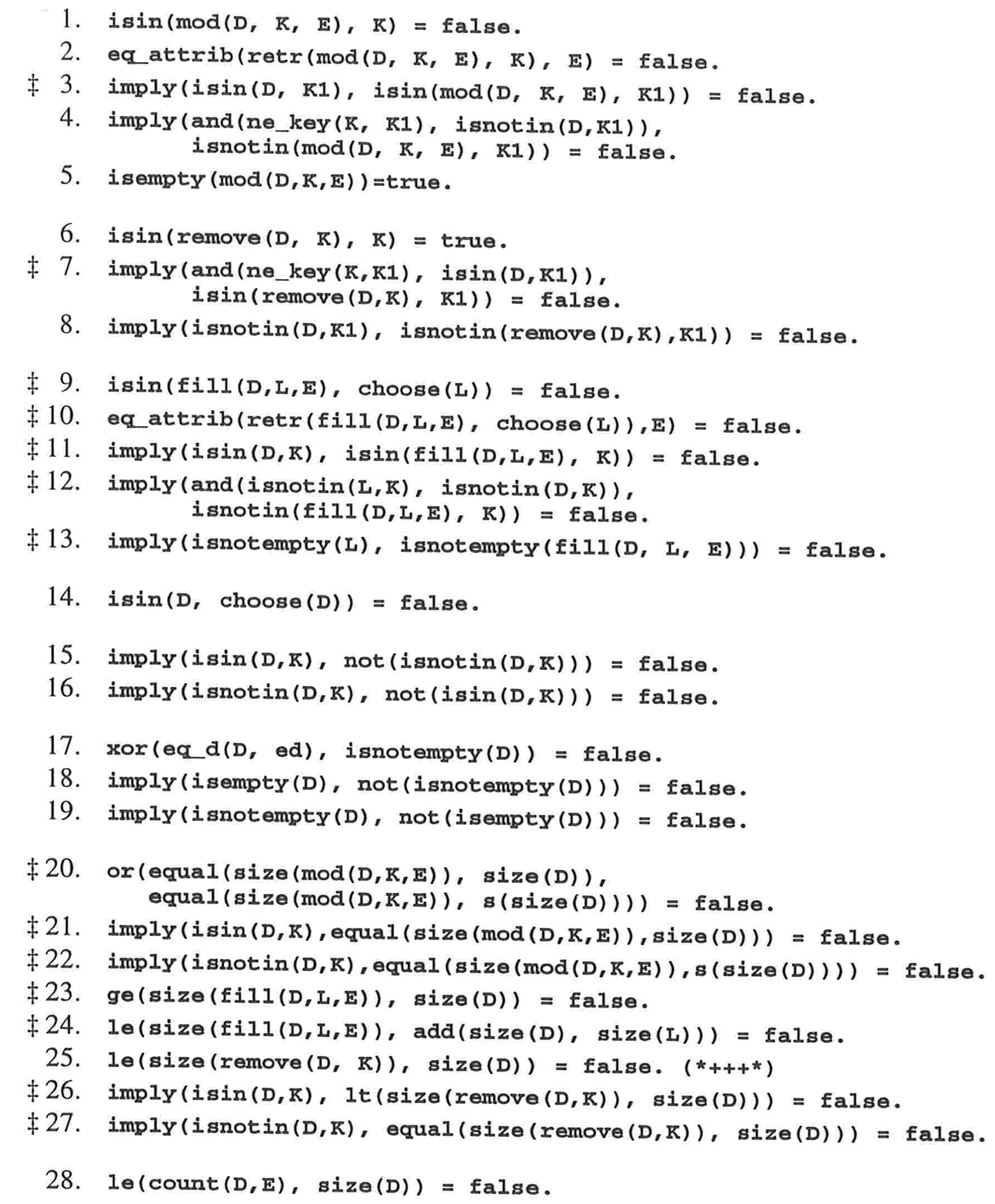




\section{Apêndice G: Manual do Usuário para GPElib}

A camada da GPE forma o nível mas baixo do Serviço de Comunicação de Grupo. A GPE fornece um mecanismo de disseminação atômica. Este método de envio de mensagens é diferente de um send simples no sentido em que o destino é um grupo de recipientes. Ou todos recebem a mensagem ou nenhum a recebe. Após a decisão, se uma mensagem difundida será confirmada ou rejetada, todos os usuários recebem a mensagem e seu resultado.

Há duas partes do sistema que um programador de uma aplicação distribuído precisa conhecer: A API fornecida por GPElib e a forma de inicialização de processos.

\section{A API GPElib}

GPElib fornece a interface para programas usuário e é muito simples, consistindo de apenas duas funções: GPE_broadcast e GPE_receive. A configuração inicial dos processos usuário com seus GPEs é tratado por estas funções. A declaração destas funções encontra-se no arquivo GPElib.h, que é mostrado abaixo.

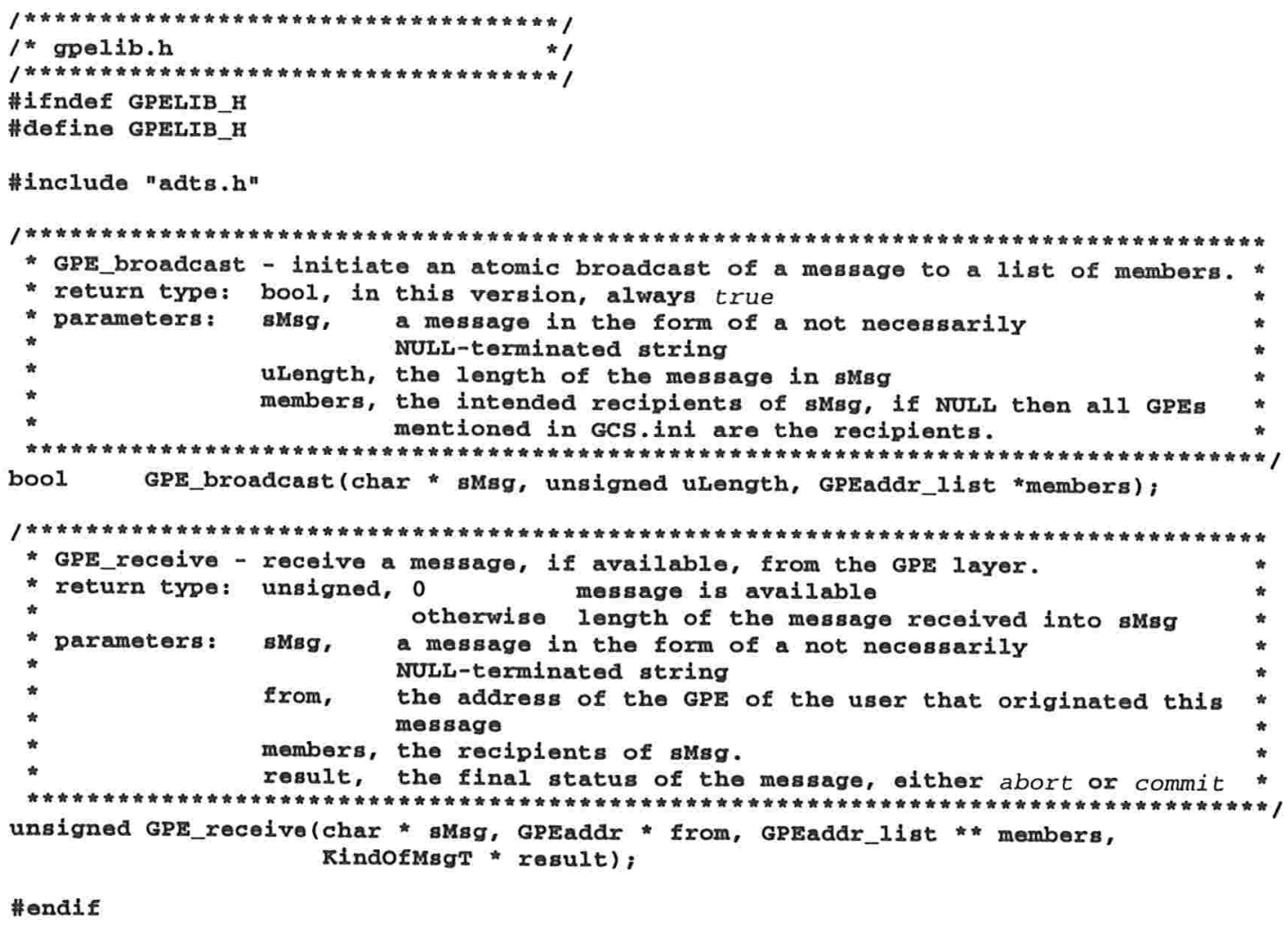

O endereço de uma GPE (como é usado nos GPEaddrs nestas duas funções) é a ordem em que são listados no arquivo GCs.ini, começando em zero. A GPEaddr_list é manipulada com as funções descritas no arquivo list.h. GPElib.o.

O código usuário é compilado com o compilador Concert/C ccc e ligado com o arquivo 
Um programa simples encontra-se na seção 3 deste apêndice.

Como descrito na especificação formal, processos usuário devem ser sempre disponível para receber uma mensagem da GPE. Isso pode ser feito incluindo-se as chamadas GPE_receive em uma thread isolado.

\section{O Processo da Inicialização das GPEs}

Antes de usar o sistema de GPEs, a configuração do sistema tem que ser descrita no arquivo GCS.ini e o caminho completo do diretório que a contém tem que estar na variável do ambiente GPEDIR. Este arquivo pode ter várias seções, mas a única de interesse para a camada de GPEs segue o cabeçalho [GPE]. Esta seção contém os valores para os time-out (em segundos) e uma lista das máquinas que serão incluídas no grupo. O broadcast time-out é o tempo de espera até que um broadcast atômico é abortado. O query time-out é o tempo de espera entre tentativas de query. Um arquivo exemplo encontra-se abaixo.

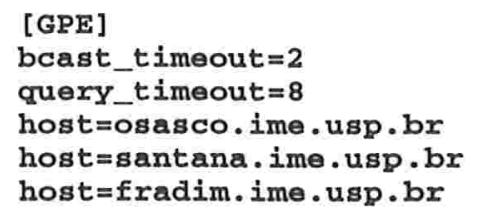

Uma vez que este arquivo contém as informações corretas, o comando launcher pode ser executado para instanciar os processos GPE nas máquinas listadas. Finalmente, os programas usuário podem ser iniciados individualmente nas suas máquinas.

\section{Um Exemplo de um Programa Usuário}

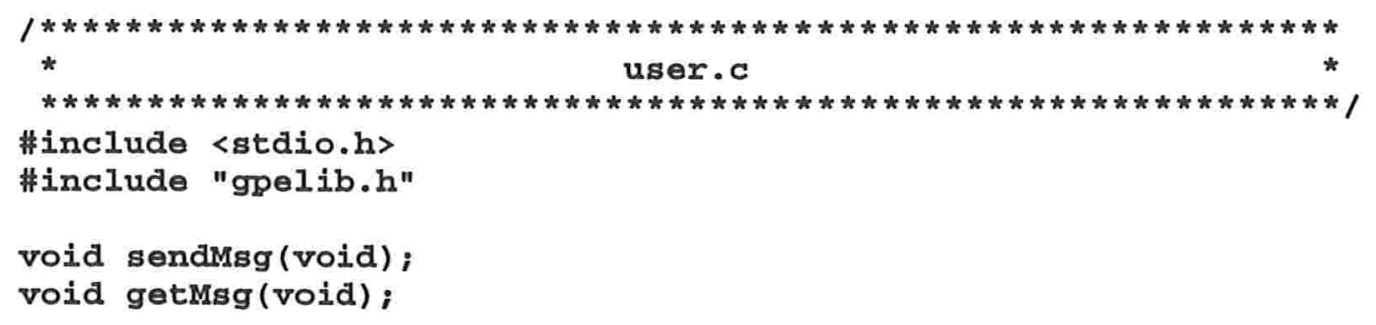




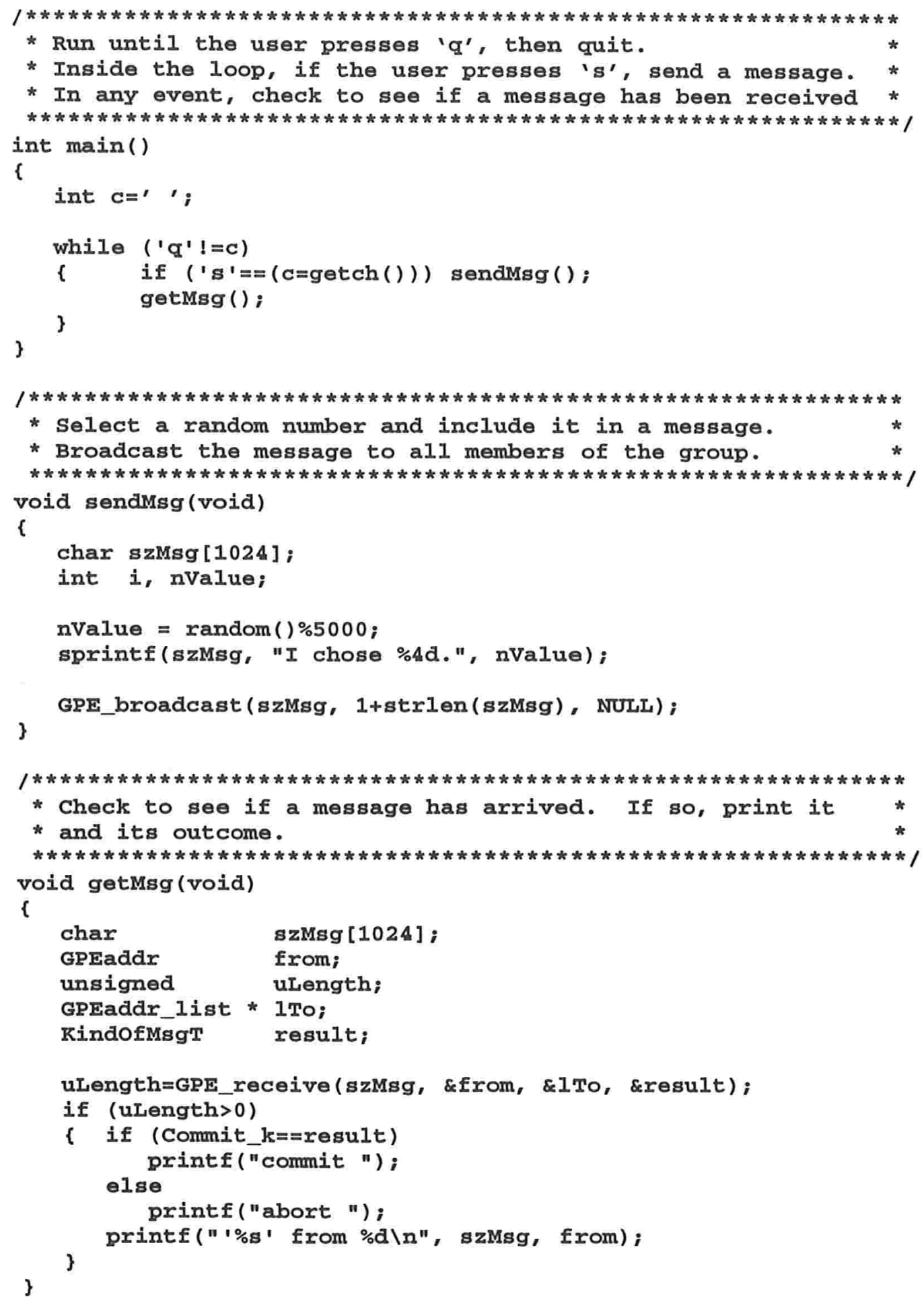




\section{Apêndice H: Parte do Arquivo list .h}

Em diversas partes da implementação utilizou-se a facilidade de macro-substituicão para criar tipos de dados similares. Para tal, utilizou-se a diretiva de pre-processamento \#\#, que é um operador pouco usado do preprocessador pcc. Kernighan and Ritchie [K\&R] mencionam que depois da substituição dos parametros, cada \#\# é excluído, como também qualquer espaço branco em volta destes simbolos, para concatenar os tokens adjacentes e formar um token novo. Como pode ser visto, existem dois macros: 1ist_decl (TYPE) que corresponde à assinatura e list (TYPE) que corresponde aos axiomas. Asserções são usados para fazer cumprir as pre- e pós-condições.

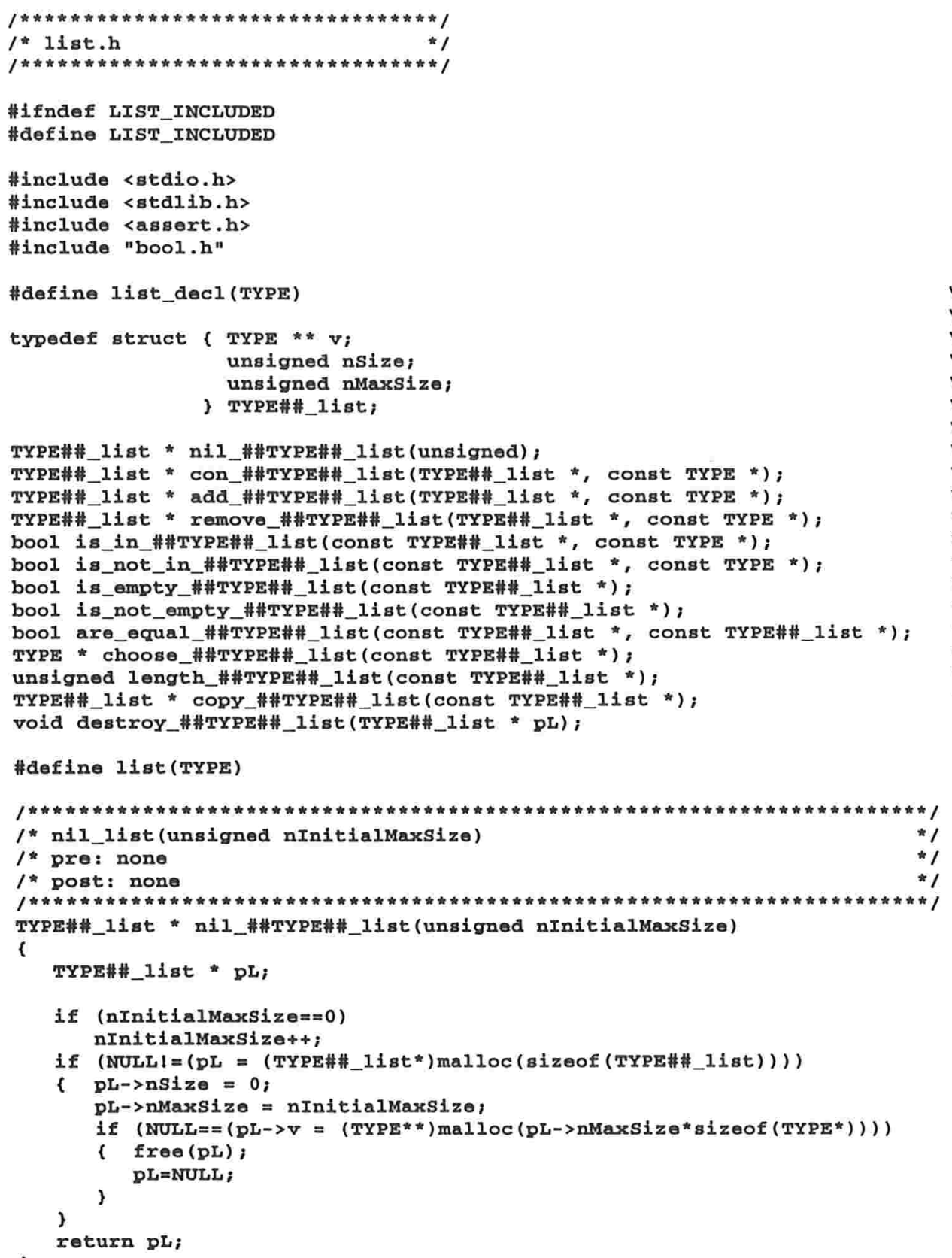




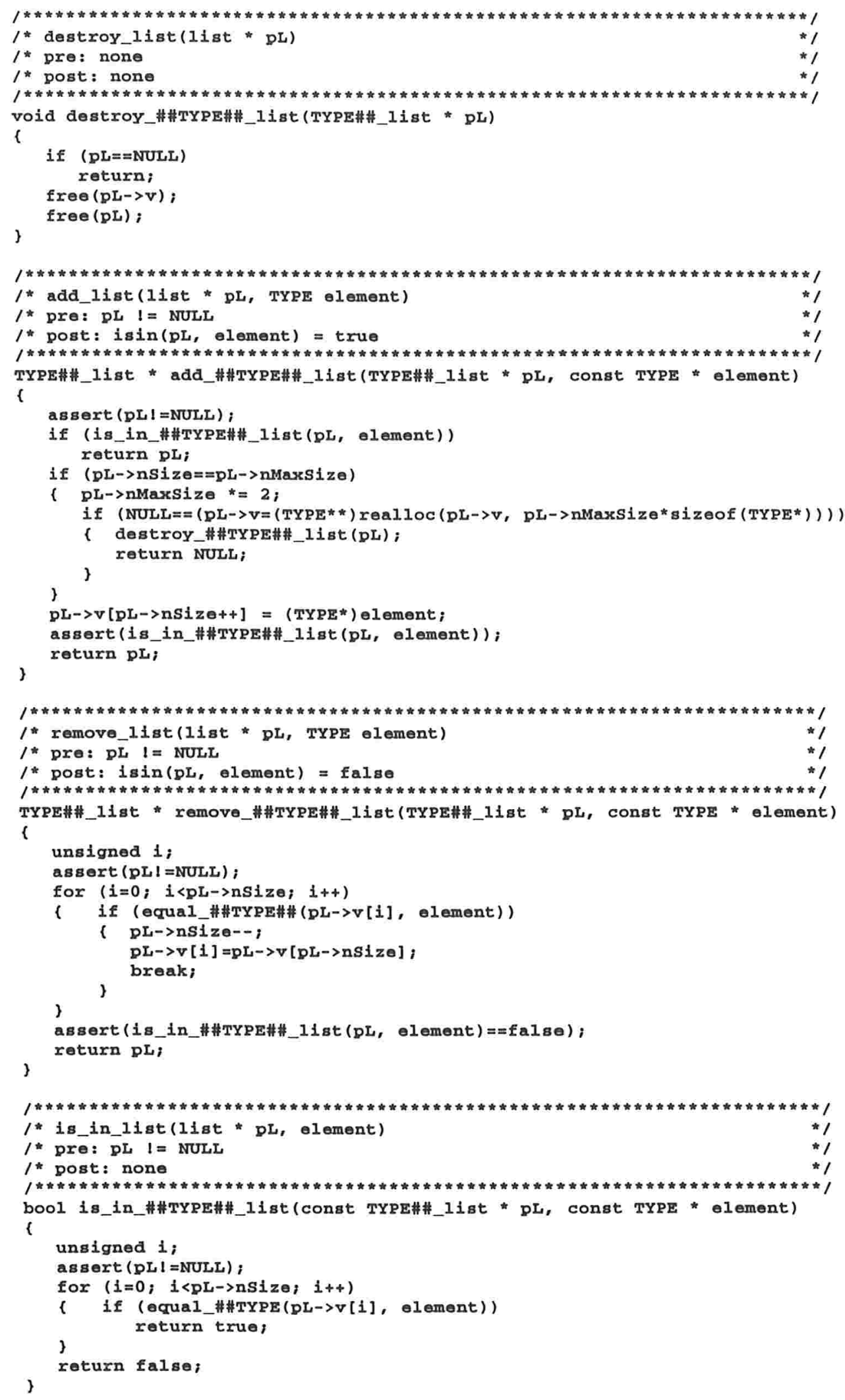




\section{Apêndice I: Parte dos Arquivos gpetypes .h e ports .h}

O arquivo gpetypes.h define, entre outras coisas, os tipos das mensagens enviadas entre processos. Os nomes dos tipos são do ponto de vista de uma GPE. Por exemplo, TouserType é o tipo de uma mensagem de um GPE para um usuário, enquanto GPEtoGPEtype é o tipo de uma mensagem entre GPEs.

$\mathrm{O}$ arquivo ports.h define, entre outras coisas, os tipos das portas que ligam os processos. Os nomes seguem o mesmo padrão do que os de gpetypes.h. Por exemplo, TouserPortType define uma porta para o envio de mensagens do tipo TouserType e é usado para ligar um usuário com seu GPE.

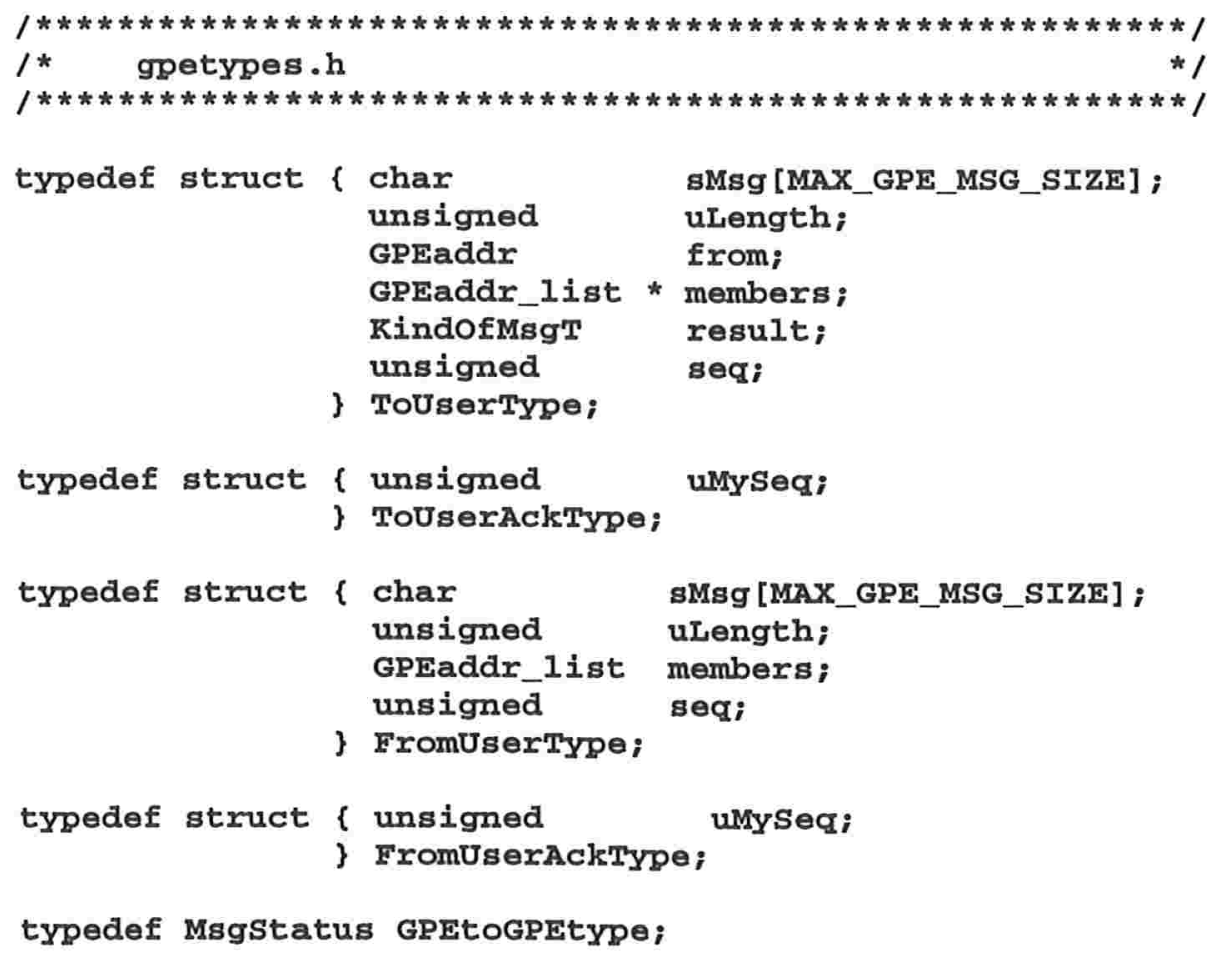




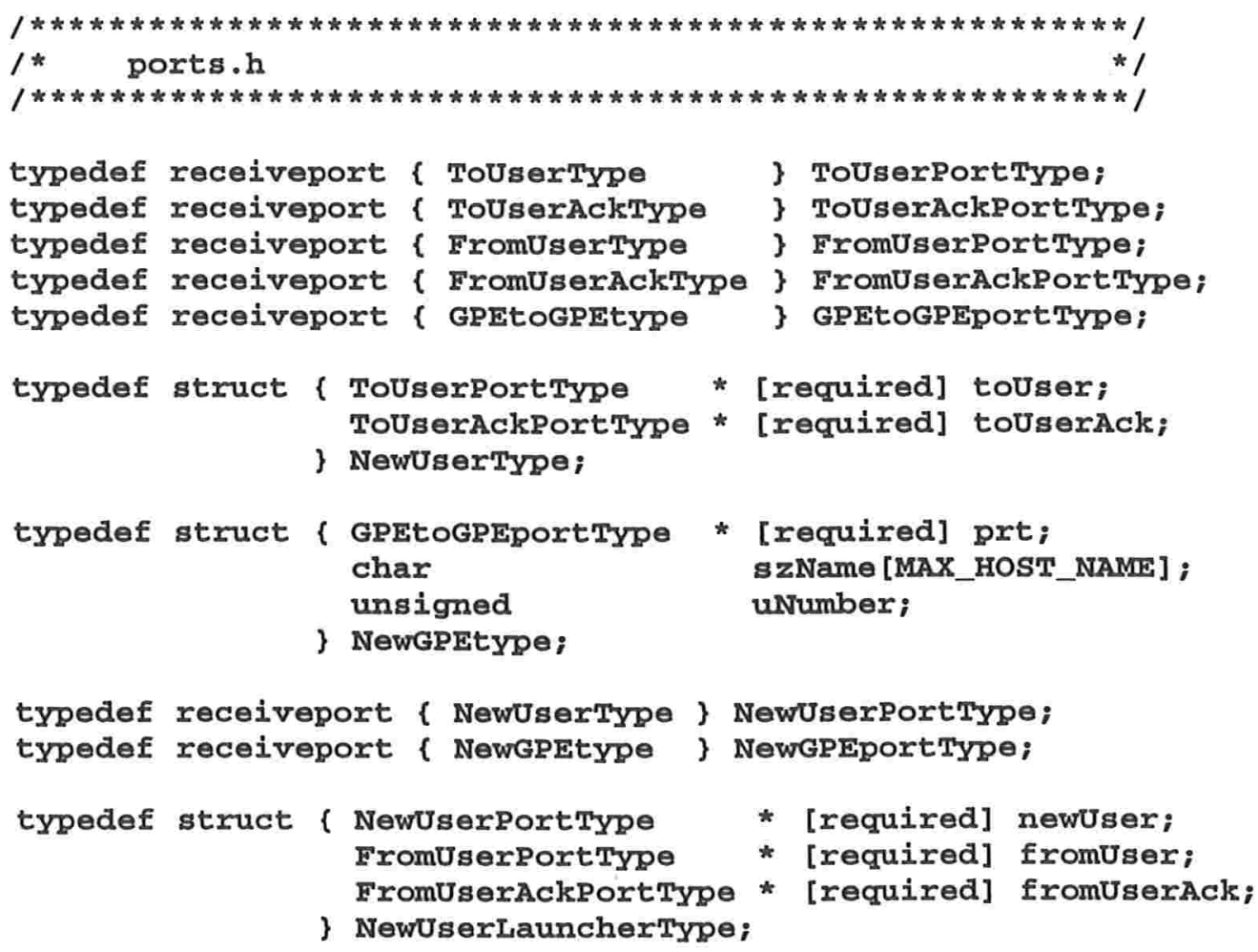




\section{Apêndice J: Parte do Arquivo gpeports .c}

O arquivo gpeports.c define os processos que implementam as ações atômicas da especificação. Escondidas aqui é todo o código que usa as extensões de Concert/C.

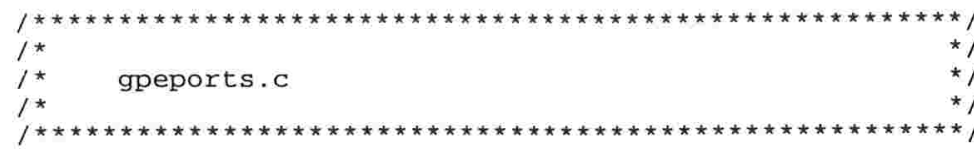

\#include <stdio.h>

\#include <stdlib.h>

\#ifdef JUST_BROADCAST

\#include "gpetypes.h"

void print_keys (GPEaddr_VoteT_dict * d)

void toGPE (const GPETOGPEtype * $\mathrm{msg}$ );

\#endif

\#ifndef JUST_BROADCAST

\#include <concert.h>

\#include "gpeports.h"

\#include "ports.h"

static GPEtoGPEportType

static TouserPortType

myGPEtoGPEport:

* myToUserPort $=$ NULL:

static ToUserAckPortType * myToUserAckPort $=$ NULL;

static FromUserPortType myFromUserPort;

static FromUserAckPortType myFromUserAckPort:

static NewGPEportType myNewGPEport;

static NewUserPortType myNewUserPort;

static NewGPEtype other_GPES [MAX_GPE_ADDRESS] ;

extern FILE * fpLog;

extern char szMyName[];

extern char szGPEdir []

extern char szLogFileName[];

static unsigned uNumGPEs;

static unsigned uBcastTimeout

static unsigned uQueryTimeOut;

\#ifdef TEST_GPE_PORTS

FILE * fpLog;

VoteT NN_V = NN_ack;

\#endif

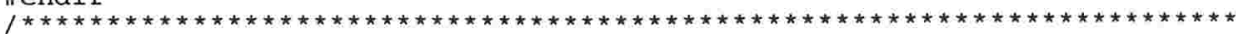

* init_port_function - called by launcher to get this GPE's port info * return type - void

parameters - GPEdir null termindated string that gives the

name of the directory containing the GCS.ini file

myName nuli terminated string that contains the

name of this GPE's host machine

numGPEs total number of GPEs in the group

bcastTO broadcast time-out value in seconds

queryTo query time-out value in seconds

gpePorts this GPE's port addresses

*

initial port

void init_port_function([in] char * GPEdir, [in] char * myName,

unsigned numGPEs, unsigned bcastTO, unsigned queryTo,

[out] GPEportListType * gpePorts)

strcpy (szGPEdir, GPEdir)

strcpy (szMyName, myName)

sprintf(szLogFileName, "zs/zs.log", szGPEdir, szMyName);

strcpy (szMyName, myName)

UNUMGPES = numGPEs :

uBcastTimeout = bcastTo;

uQuerYTimeout = querYTO;

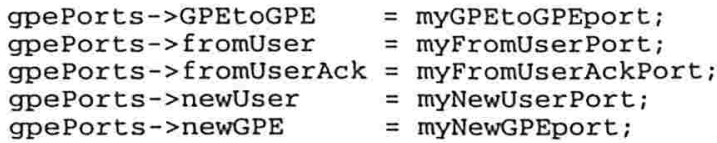


fpLog $=$ fopen (szLogFileName, "a");

fprintf (fplog, "8s is aliveln", szMyName);

fclose (fpLog)

\begin{tabular}{|c|c|c|c|c|}
\hline * & $\begin{array}{l}\text { connect_GPEs - } \\
\text { return type }\end{array}$ & $\begin{array}{l}\text { called by } I \\
\text { _ void }\end{array}$ & main to send GPE port info to all GPEs & * \\
\hline * & parameters & - szGPEdir & null termindated string that gives the & * \\
\hline * & & & $\begin{array}{l}\text { name of the directory containing the } \\
\text { GCS ini file }\end{array}$ & * \\
\hline * & & puNumGPEs & $\begin{array}{l}\text { GCS.inl tile } \\
\text { total number of GPEs in the group }\end{array}$ & * \\
\hline * & & bcastTo & broadcast time-out value in seconds & * \\
\hline * & & queryTo & query time-out value in seconds & * \\
\hline
\end{tabular}

void connect_GPEs (char * szGPEdir, unsigned * puNumgPEs,

\{

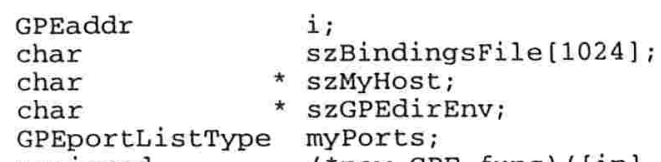

char * szMyHost;

char * szGPEdirEnv;

GPEportListType myPorts;

unsigned (*new_GPE_func) ([in] GPEportListType, unsigned * uQueryTO,

\#ifdef TEST_GPE_PORTS

GPEtoGPEtype Hello;

\#endif

if $\left({ }^{\prime} \backslash 0^{\prime}==\right.$ szGPEdir $\left.[0]\right)$

\{ accept(init_port_function):

${ }^{*}$ puNumGPEs $=$ uNumGPEs ;

${ }^{\star}$ puBcastTO $=$ uBcast $\mathrm{T}$ imeOut

* puQueryTo $=$ uQueryTimeOut

else

( szMyHost $=$ getenv ( HOST"):

szGPEdirEnv = getenv ("GPEDIR")

strcpy (szMyName, szMyHost);

strcpy (szGPEdir, szGPEdirEnv);

free (szMyHost);

free (szGPEdirEnv)

sprintf(szBindingsFile, "\%s/gpe_bindings_file", szGPEdir)

switch (cn_shared_file_import(szBindingsFile, "new_GPE_func", \&new_GPE_func))

\{ case cn_success : break;

case cn_resource_not_available :

fpLog = fopen (szLogFileName, "at")

fprintf (fpLog, "resource not available: file 'os' not found $1 n$ ", szBindingsFile)

fclose (fpLog);

exit $(0)$;

case cn_symbol_not_available :

fpLog = fopen (szLogFileName, "at")

fprintf(fpLog, "symbol not available: symbol 'new_GPE_func' not found in file 'os' $\mathrm{f}$ ", szBindingsFil. fclose (fpLog); exit (0);

case cn interface mismatch

fpLog = fopen (szLogFileName, "at").

fprintf(fpLog, "interface mismatch for new_GPE_func in binding file $\backslash n$ ");

fclose (fpLog);

exit (0):

myPorts.GPEtoGPE = \&myGPEtoGPEport $;$

myPorts.fromuser = \&myFromuserPort;

myPorts. fromUserAck $=$ \&myFromUserAckPort;

myPorts. newUser $\quad=$ \&myNewUserPort

myPorts. newGPE $\quad$ \&myNewGPEport

strcpy (myPorts.szName, szMyName);

${ }^{*}$ puNumGPEs = new_GPE_func (myPorts, puBcastTo, puQueryTo);

for ( $i=0$; $i<$ NumGPEs; $i++)$

\{ receive(myNewGPEport, \& (other_GPEs[i])):

\#ifdef TEST GPE_PORTS

strcpy (Hello.data, "Hello there!")

Hello. coord $=0$

Hello. to $=0$;

Hello. from $=0$;

Hello. $\mathrm{kind}=0$;

Hello.acks $=$ NULL

for $(i=0 ; i<U N U m G P E s ; i++)$

( if (other_GPEs [i].prt $==$ myGPEtoGPEport)

$\{$ fpLog $=$ fopen (szLogFileName, "a");

fprintf (fpLog, "os is number od $\backslash$ n", szMyName, i) ;

) fclose (fpLog) :

else 


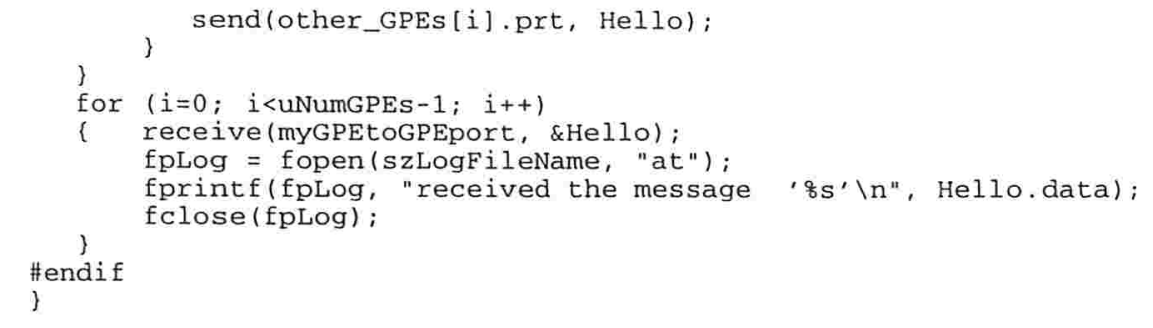




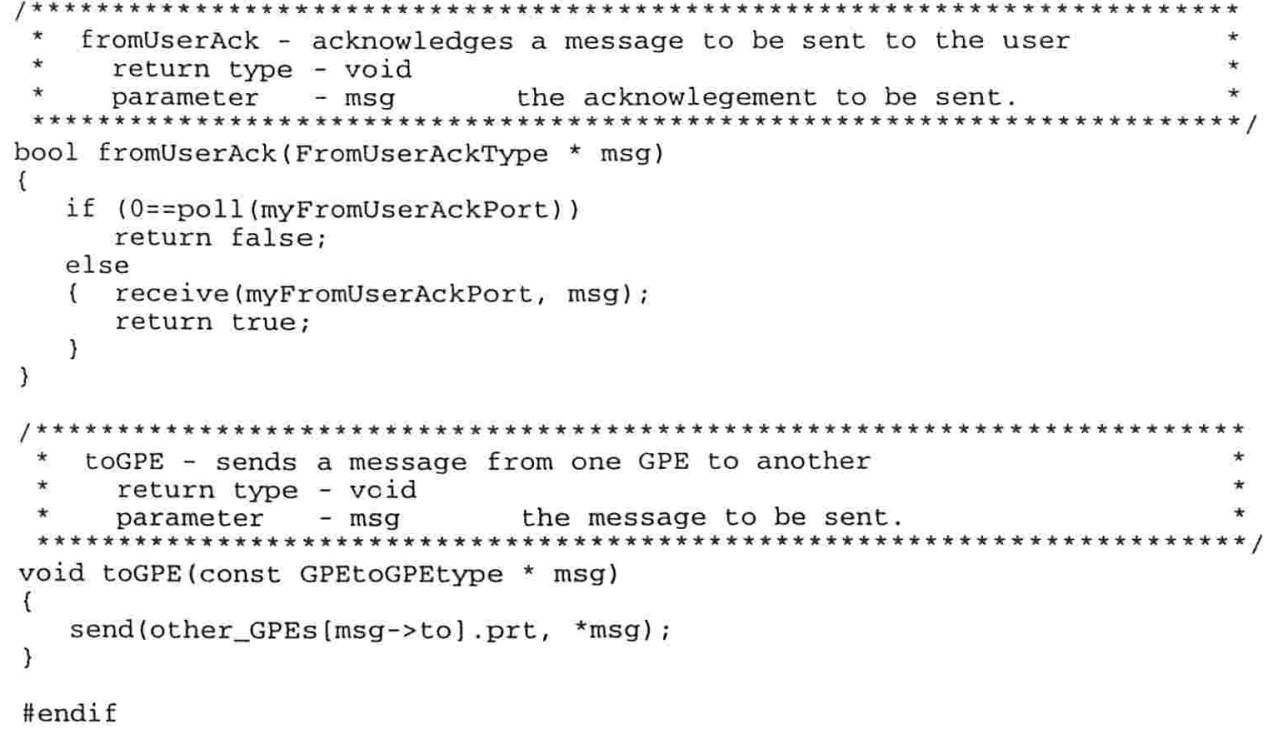




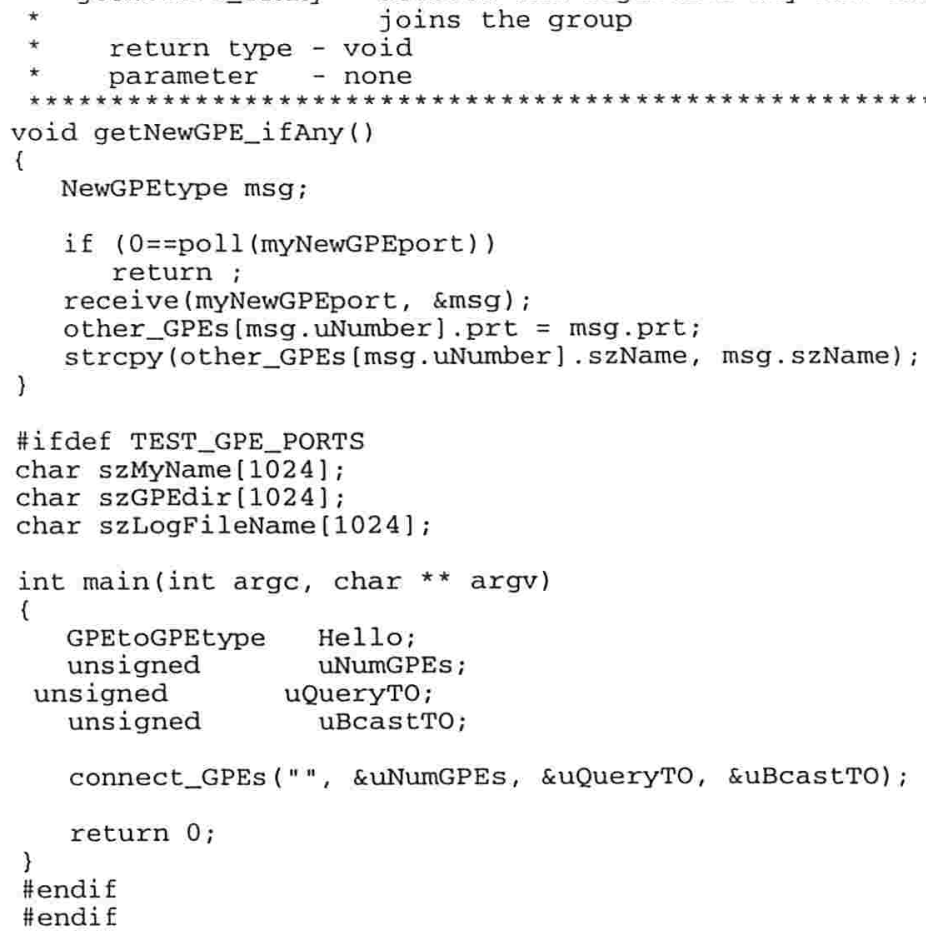




\section{Apêndice K: Parte do arquivo gpemisc.c}

As funções definidas neste arquivo implementam a maioria dos processos descritos na especificação formal.

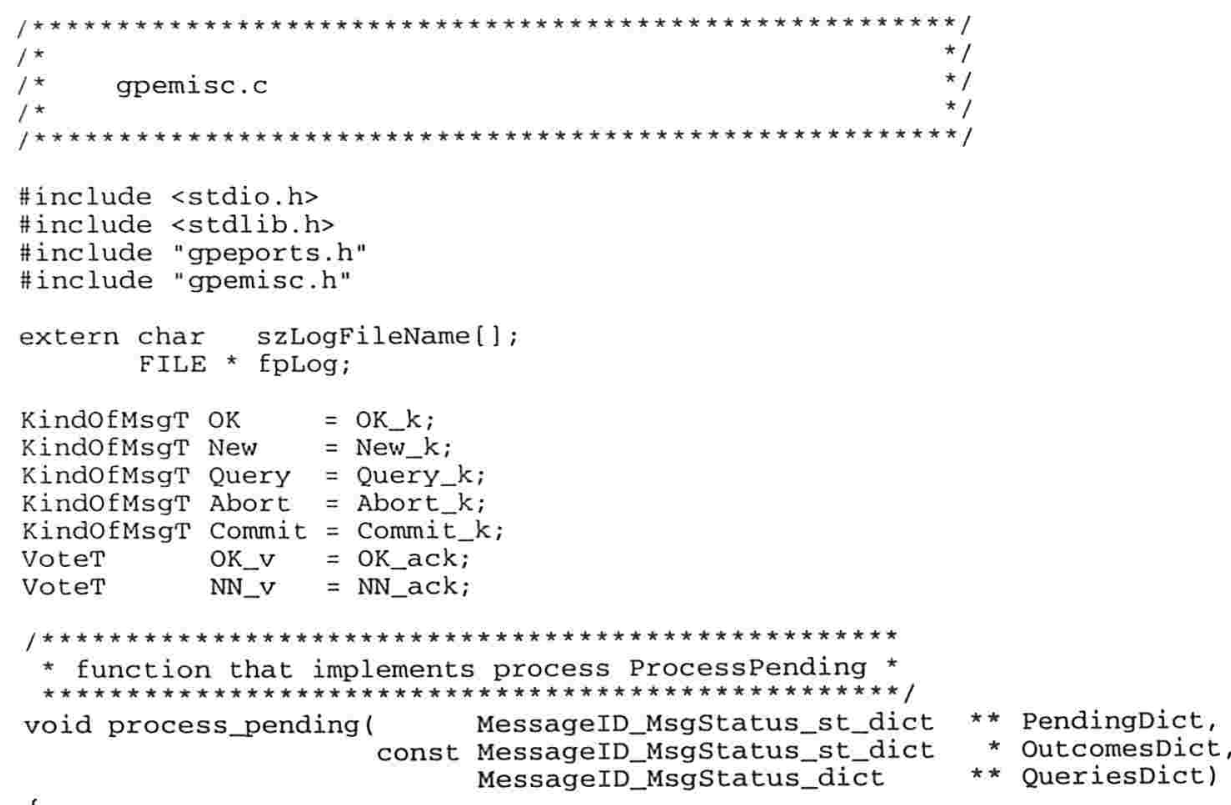

(

MessageID_list * keyList;
MessageID * pendKey;
MsgStatus * pendAttrib;
MsgStatus * outcomeAttrib;

keyList $=$ get_keys_MessageID_Msgstatus_st_dict (*PendingDict) $;$

while (is_not_empty_MessageID_list(keyList))

\{ pendKey $=$ choose_MessageID_list (keyList) : pendAttrib = retr_MessageID_MsgStatus_st_dict (*PendingDict, pendKey);

switch (pendAttrib->kind)

$\{$ case OK_k : *QueriesDict $=$ mod_MessageID_Msgstatus_dict (*QueriesDict, pendKey, pendAttrib) ; pendAttrib->kind $=$ Query_k; broadcast_to_GPEs (pendAttrib): pendAttrib->kind $=$ OK_k; break;

case New_k : if (true==is_in_MessageID_MsgStatus_st_dict(OutcomesDict, pendKey))

\{ outcomeAttrib = retr_MessageID_Msgstatus_st_dict(OutcomesDict, pendKey); pendAttrib->kind $=$ outcomeAttrib->kind broadcast_to_GPEs (pendAttrib) ; touser (pendattrib); *PendingDict = remove_MessageID_MsgStatus_st_dict(*PendingDict, pendKey) ; destroy_MessageID (pendKey) :

\} destroy_MsgStatus (pendattrib);

else

\{ broadcast_to_GPEs (pendAttrib) ; set_all_acks (pendAttrib, \&NN_v)

break

default : fplog = fopen(szLogFileName, "a"):

fprintf(stderr, "Pending has a record of unknown type $\backslash \mathrm{n}$ ");

fclose (fpLog):

\} break

keyList $=$ remove_MessageID_list (keyList, pendKey) $;$

destroy_MessageID_list(keyList); 


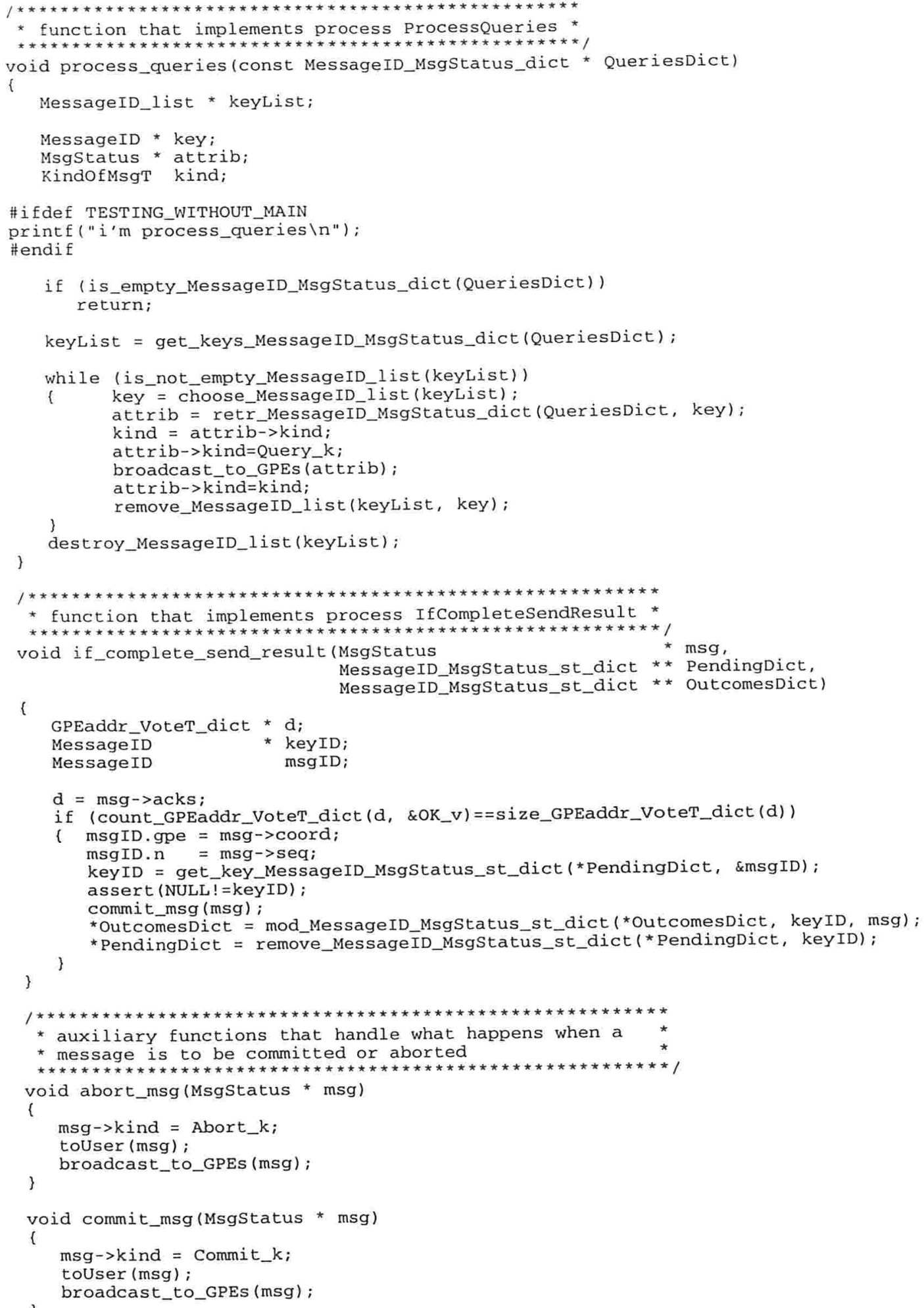




\section{Apêndice L: Parte do arquivo gpemain.c}

As funções deste arquivo implementam os processos Recover e GroupProtocol descritos na especificação formal. Também neste arquivo encontra-se a função principal do GPE que interliga-se com as demais GPEs.

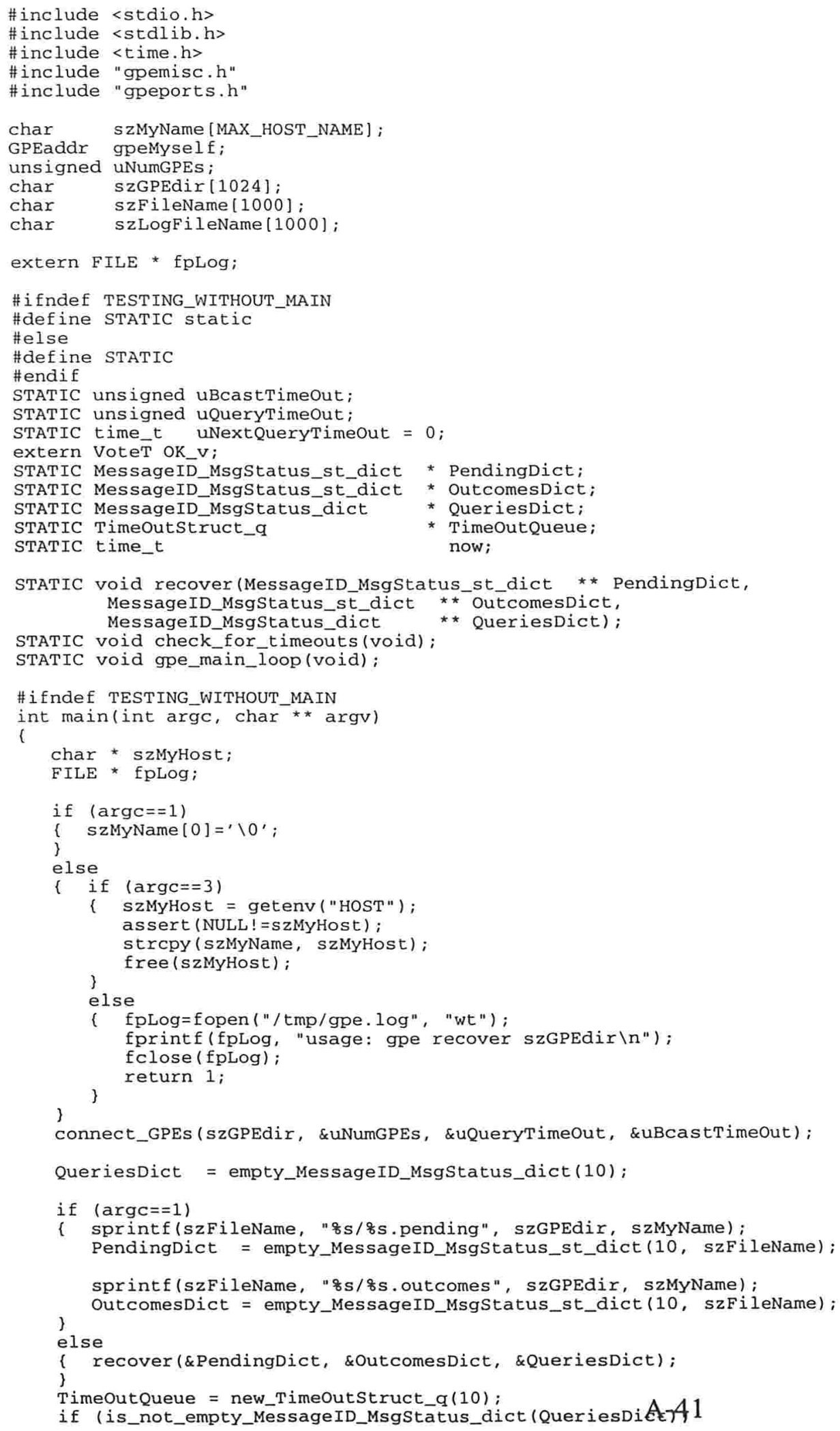


fpLog $=$ fopen (szLogFileName, "a") ;

fprintf(fpLog, "ss going to main loop in", szMyName);

fclose (fpLog);

gpe_main_loop () :

\#endif

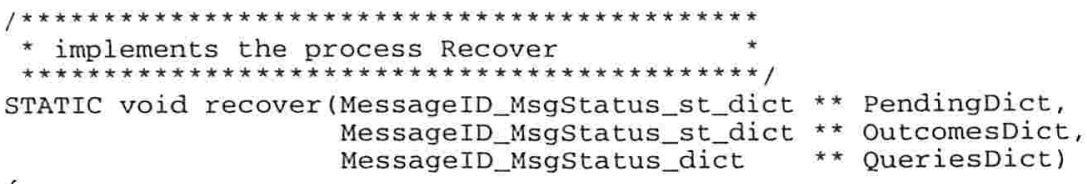


toRecord->msg = msg;

TimeoutQueue = add_TimeoutStruct_q(TimeoutQueue, toRecord)

msg=NULL;

continue; $I^{*}$ cannot process a possible message from the net until

i msg is reallocated at the top of the loop */

fpLog $=$ fopen (szLogFileName, "a");

fprintf(fpLog, "got message' ' $8 s^{\prime}$ ' of type $z d$ from gpe $8 d \backslash n "$, msg->data, msg->kind, msg->from); fclose (fplog);

I*

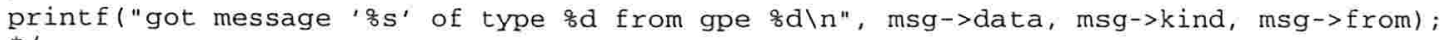
*

mid->gpe $=$ msg $->$ coord;

mid $\rightarrow$ n $=$ msg $\rightarrow$ seq

switch (msg->kind)

i case OK_k:

if (is_in_MessageID_Msgstatus_st_dict(PendingDict, mid))

( msgorig = retr_MessageID_Msgstatus_st_dict (PendingDict, mid); msgorig->acks = mod_GPEaddr_VoteT_dict (msgorig->acks, \&msg->from, \&OK_v); if_complete_send_result(msgorig, \&PendingDict, \&OutcomesDict);

case New_k: $\quad$ msg->kind=oK_k;

msg $\rightarrow$ from=gpeMyself

$\mathrm{msg} \rightarrow$ to $=\mathrm{msg} \rightarrow$ coord;

mod_MessageID_Msgstatus_st_dict(PendingDict, mid, msg)

tOGPE (msg);

$\mathrm{msg}=\mathrm{NULL}$;

break;

case Commit_k:

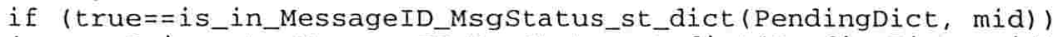

$\{$ msgorig=retr_MessageID_MsgStatus_st_dict (PendingDict, mid); msgorig->kind $=$ Commit_k;

mod_MessageID_MsgStatus_st dict(OutcomesDict, mid, msgorig) : remove_MessageID_Msgstatus_dict(QueriesDict, mid);

msgorig $\rightarrow$ from $=$ msgorig $\rightarrow$ coord;

touser (msgorig);

remove_MessageID_Msgstatus_st_dict(PendingDict, mid) ;

case Abort_k:

if (true==is_in_MessageID_MsgStatus_st_dict (PendingDict, mid))

( msgorig=retr_MessageID_MsgStatus_st_dict(PendingDict, mid) msgorig->kind=Abort_k;

mod_MessageID_MsgStatus_st_dict(OutcomesDict, mid, msgorig) remove_MessageID_MsgStatus_dict(QueriesDict, mid);

msgorig $\rightarrow$ from=msgorig->coord;

remove_MessageID_MsgStatus_st_dict(PendingDict, mid) ;

\}

touser (msgorig);

if (true==is_in_MessageID_Msgstatus_st_dict (OutcomesDict, mid))

$\{$ msgorig=retr_MessageID_Msgstatus_st_dict(OutcomesDict, mid) ; msg $\rightarrow$ kind $=$ msgorig $\rightarrow$ kind; msg $\rightarrow$ to $=$ msg $\rightarrow$ from;
msg $\rightarrow$ from $=$ gpeMyself tOGPE (msg) ;

break;

STATIC void check_for_timeouts(void)

\{

Timeoutstruct * qe_first;
MessageID * midorig, mid;

time (\&now)

if (is_empty_Timeoutstruct_q(TimeoutQueue))

return;

qe_first $=$ first_TimeOutStruct_q(TimeOutQueue):

while (qe_first!=NULL \&\& now $>=$ qe_first $\rightarrow$ UTO)

( if (qe_first $\rightarrow$ msg $\rightarrow>$ kind $==N$ New_k)

( mid.gpe $=$ ge_first $\rightarrow$ msg $->$ coord ;

mid.n $=$ qe_first $>$ msg->seq;

midorig = get_key_MessageID_Msgstatus_st_dict (PendingDict, \&mid);

qe_first $\rightarrow$ msg $->$ kind $=$ Abort_k;

OutcomesDict = mod_MessageID_Msgstatus_st_dict(OutcomesDict, midorig, qe first->msg); abort_msg(qe_first->msg): remove_MessageID_MsgStatus_st_dict(PendingDict, midorig) :

TimeOutQueue = remove_TimeOutStruct_q(TimeOutQueue $)$;

free (qe_first) :

if (is_empty_Timeoutstruct_q(TimeoutQueue))

else

e_first $=$ NULL;

qe_first $=$ first_TimeOutstruct_q(TimeOutQueue);

while (qe_first!=NULL \&\& qe_first->msg->kind!=New_kA-43 
( TimeoutQueue $=$ remove_TimeOutstruct_q(TimeoutQueue);

free (qe_first);

if (is_empty_Timeoutstruct_q(TimeoutQueue))

qe_first $=$ NULL:

else

qe_first $=$ first_Timeoutstruct_q(TimeoutQueue) $;$ 


\section{Apêndice M: Parte do arquivo launcher.c}

O launcher é instancia as GPEs nas máquinas listadas no arquivo Gcs.ini. Também tem a responsabilidade de interligar as GPEs e fornecer os endereços das portas delas para os processos de usuários.

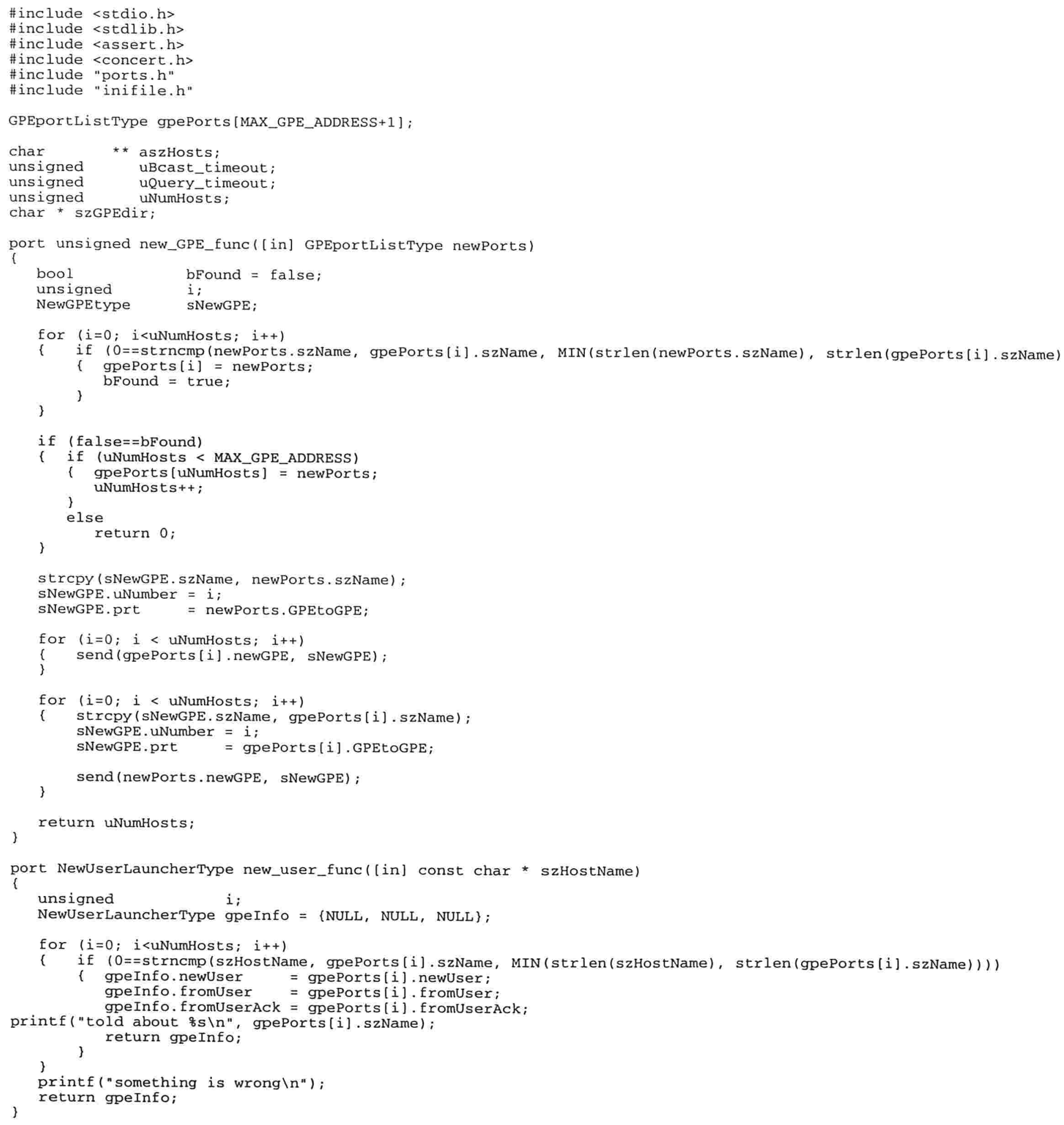


11

use shared file:

export new_GPE_func ( to "./gpe_bindings_file" \};

1]

export new user func (to "/gpe bindings file" ; ;

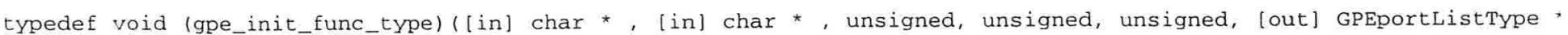
int main(void)

\{

unsigned $i, j$;

char szCommand [1024].

program prog gpe [MAX GPE_ADDRESS+1];

gpe_init_func_type *gpe_binding [MAX_GPE_ADDRESS+1] ;

NewGPEtype startinfo;

if (NULL $==($ szGPEdir=getenv ("GPEDIR")) )

( printf ("must define GPEDIR in the environment $\backslash \mathrm{n}$ "); exit (EXIT_FAILURE)

printf("gpedir is os $\backslash \mathrm{n}$ ", szGPEdir)

uNumHosts = read_inifile("GCS.ini", \&aszHosts, \&uBcast_timeout, \&uQuery_timeout);

assert ((uNumHosts $>\overline{0}$ ) \&\& (UNumHosts<=MAX_GPE_ADDRESS)) ;

printf("the of hosts are $\backslash \mathrm{n} "$, uNumHosts);

for ( $i=0$; $i<u$ unumosts; $i++)$

printf("'\%s' $\backslash n "$, aszHosts [i]):

for $(i=0 ; i<$ unumHosts; $i++)$

( prog_gpe[i] = cn_prog_init ();

sprintf( szCommand, "/usr/X11R5/bin/xterm-display $\% s$-title gpe@s "

"-e /home/posmac/cd/mon/mon4/gpe/gpe concert_args termio",

getenv ("DISPLAY"), aszHosts[i])

cn_prog_set_name (prog_gpe[i], szCommand) ;

cn_prog_set_host(prog_gpe[i], aszHosts[i]):

if (cn_executable_not_found $==$ cn_prog_set_ptty (prog_gpe[i], "xhost", 0))

( perror ("couldn't find executable") exit(EXIT_FAILURE)

if (cn_executable_not_found $==\mathrm{cn}$ prog_set_relative_name (prog_gpe[i], " /home/posmac/perry/cd/mon/mon4/gpe/gpe ( perror("couldn't find executable");

了 exit (EXIT_FAILURE);

create (prog_gpe[i], (gpe_init_func_type **) \& (gpe_binding[i])) ;

for $(i=0 ; i<$ uNumHosts; $i++)$

( gpe_bindingli] (szGPEdir, aszHosts[i], uNumHosts, uBcast_timeout, uQuery_timeout, \&(gpePorts[i]));

for $(i=0 ; i<$ uNumHosts; $i++)$

f for $(j=0 ; j<$ unumHosts; $j++)$

\{ strcpy(startinfo.szName, gpePorts[j].szName); start Info. uNumber $=j$;

startInfo.prt $=$ gpePorts $[j]$. GPEtoGPE

send(gpePorts [i]. newGPE, startInfo);

while (1)

accept (new_GPE_func, new_user_func) ; 


\section{Bibliografia}

[Arn] Arnold, A., M.C. Gaudel, and B. Marre. "An Experiment on the Validation of a Specification by Heterogeneous Formal Means: The Transit Node." Fifth International Working Conference on Dependable Computing for Critical Applications, 1995.

[Ar2] Arnold, A. Finite Transition Systems. Prentice Hall, 1994.

[Aue] Auerbach, Joshua S. et al. Concert/C Tutorial and User's Guide: An Introduction to a Language for Distributed C Programming. Hawthorne, New York: IBM T. J. Watson Research Center, 1995.

[Ber] Bernot, G., M.C. Gaudel and B. Marre. "Software testing based on formal specifications: a theory and a tool." Software Engineering Journal. 6 (6): November 1991.

[Bir] Birman, Kenneth P. Building Secure and Reliable Network Applications. Greenwich : Manning, 1996.

[Biv] Birman, Kenneth P, and Robert van Renesse. Reliable Distributed Computing with the Isis Toolkit. Los Alamitos, CA: IEEE Computer Society Press, 1994.

[Bri] Brinksma, Ed. "A Theory for the Derivation of Tests." Protocol Specification, Testing, and Verification VIII. North-Holland: Elsevier Science Publishers, 1988.

[Cam] Campos, S., E. M. Clarke, M. Minea, "Symbolic Techniques for Formally Verifying Industrial Systems." Science of Computer Programming.

29 (1-2), 79-98, 1997.

[Can] Caneve, Maurizio, and Elena Salvatori, eds. Lite User Manual. 1992. http://www.inria.fr/meije/lotostools.html

[Cla] Clarke, E. M. E. A. Anderson, and A. P. Sistla. "Automatic verification of finite-state concurrent systems using temporal logic specifications." ACM Transactions on Programming Languages and Systems, 8(2), 244-263, 1986.

[Cla] Claßen, Ingo, Hartmut Ehrig, and Dietmar Wolz. Algebraic Specification Techniques and Tools for Software Development: The ACT Approach. River Edge, New Jersey: World Scientific Publishing Co. Pte. Ltd., 1993.

[End] Endler, Markus, and Anil D'Souza. "Supporting Distributed Application Management in Sampa." Technical report of the Departamento de Ciência da Computação, Instituto de Matemática e Estatística, Universidade de São Paulo. 1995. 
[Fan] Fantechi, Alessandro, et al. "Towards Automatic Temporal Logic Verification of Value Passing Process Algebra Using Abstract Interpretation." 7th International Conference on Concurrency Theory. Ed. Ugo Montanari and Vladimiro Sassone. Springer-Verlag, 1997. 565-578.

[Gau] Gaudel, Marie-Claude. "Testing can be formal, too." Ed. Peter D. Mosses et al. TAPSOFT '95: Theory \& Practice of Software Development. Lecture Notes in Computer Science 915, Springer-Verlag, 1995. 82-96.

[Gor] Gordon, Michael, J. C., The Denotational Description of Programming Languages: An Introduction. New York: Springer-Verlag, 1979.

[Had] Hadzilacos, Vassos and Sam Toueg. "Fault-Tolerant Broadcasts and Related Problems." Distributed Systems, 2nd ed. Ed. Sape Mullender. New York: ACM Press, 1993.

[Hen] Hennessy, Matthew. Algebraic Theory of Processes. Cambridge, Mass.: The MIT Press, 1988.

[Hoa] Hoare, C. A. R. "An Axiomatic Basis for Computer Programming." Communications of the ACM. 12 (1969): 576-583.

[Hol] Holzmann, Gerard J. Design and Validation of Computer Protocols. Englewood Cliffs, N.J.: Prentice-Hall, 1991.

[Iso] ISO. Information Processing Systems, Open Systems Interconnection, LOTOS: A Formal Description Technique Based on the Temporal Ordering of Observational Behaviour. IS-8807. Geneva: ISO, 1989.

[K\&R] Kernighan, Brian W., and Dennis M. Ritchie. The C Programming Language, 2nd edition. Editora Prentice-Hall do Brasil, Ltda.: Rio de Janeiro, 1988.

[Man] Manna, Zohar, and Amir Pnueli. The Temportal Logic of Reactive and Concurrent Systems. New York: Springer-Verlag, 1992.

[Tan] Tanenbaum, Andrew S. Distributed Operating Systems. Englewood Cliffs, N.J.: Prentice Hall, 1995.

[Tre] Tretsman, J. "Test Generation with Inputs, Outputs, and Quiescence.” Ed. T. Margaria and B. Steffen. Second International Workshop on Tools and Algorithms for the Construction and Analysis of Systems, pages 127-156. Lecture Notes in Computer Science 1055, Springer-Verlag, 1996. 